\title{
Absolute instabilities of standing pulses
}

\author{
Björn Sandstede \\ Department of Mathematics \\ The Ohio State University \\ Columbus, OH 43210, USA
}

\author{
Arnd Scheel \\ Department of Mathematics \\ University of Minnesota \\ Minneapolis, MN 55455, USA
}

July 26, 2004

\begin{abstract}
We analyse instabilities of standing pulses in reaction-diffusion systems that are caused by an absolute instability of the homogeneous background state. Specifically, we investigate the impact of pitchfork, Turing and oscillatory bifurcations of the rest state on the standing pulse. At a pitchfork bifurcation, the standing pulse continues through the bifurcation point where it selects precisely one of the two bifurcating equilibria. At a Turing instability, symmetric pulses emerge that are spatially asymptotic to the bifurcating spatially-periodic Turing patterns. These pulses exist for any wavenumber inside the Eckhaus stability band. Oscillatory instabilities of the background state lead to genuinely time-periodic pulses that emit small wave trains with a unique selected wavenumber. We analyse these three bifurcations by studying the standingwave and modulated-wave equations: In this setup, pulses correspond to homoclinic orbits to equilibria that undergo reversible bifurcations. We use blow-up techniques to show that the relevant stable and unstable manifolds can be continued across the bifurcation point and to investigate both existence and stability of the bifurcating waves.
\end{abstract}

\section{Introduction}

Standing pulses arise in many reaction-diffusion systems, and it is therefore natural to investigate the bifurcations that occur when they destabilize. Since the stability properties of localized pulses are determined entirely by the spectrum of the linearization, pulses destabilize precisely when part of the associated spectrum crosses the imaginary axis. On unbounded domains, the spectrum of pulses is the union of the point spectrum, which by definition consists of all isolated eigenvalues with finite multiplicity, and the essential spectrum. If isolated eigenvalues cross the imaginary axis, then the resulting bifurcations correspond to standard steady-state bifurcations such as folds, pitchforks or Hopf bifurcations. In particular, the dynamics can be described by the finite-dimensional normal form for the specific steady-state bifurcation that couples to an equation for the position or the speed of the pulse $[22,23]$. In contrast, if essential spectrum crosses the imaginary axis, finite-dimensional reductions may not be available. The essential spectrum is, however, determined entirely by the asymptotic homogeneous background state of the pulse. The resulting instability typically creates 
small-amplitude spatially-periodic patterns at the tails of the pulse which then interact with the localized pulse. It is this interaction that any bifurcation theory for essential spectra has to take into account.

In earlier work $[16,17,19]$, we have investigated bifurcations from travelling waves that are caused by the essential spectrum. The fact that the pulses move with nonzero speed renders the instability convective in nature [18, 19]: Even though perturbations grow in overall amplitude, they are outrun by the travelling pulse so that their amplitude decays pointwise in a frame that moves with the pulse. Standing pulses, on the other hand, have vanishing speed, so that essential instabilities have a more severe effect on the pulse since perturbations grow pointwise and cannot be outrun by the wave. The latter scenario is often referred to as an absolute instability. As argued below, absolute instabilities are more difficult to investigate.

In this paper, we focus on three different scenarios that occur when essential spectrum crosses the imaginary axis as $\mu$ increases through zero. First, essential spectrum may cross the imaginary axis away from zero which therefore corresponds to an oscillatory instability. In the other two cases, the essential spectrum crosses at the origin, thus causing a bifurcation either to a homogeneous steady state via, for instance, a pitchfork bifurcation or else to a Turing pattern via a Turing bifurcation.

To analyse these bifurcations, we seek pulses as solutions to the standing-wave (or modulated-wave) equation. In contrast to our earlier work [16, 17, 19], the center manifolds that we will encounter have dimension four instead of two. In addition, pulses at pitchfork and Turing bifurcations will typically decay algebraically and not exponentially. To resolve these issues, we use blow-up techniques to regularize the flow on the center manifold and to take the algebraic decay of the pulses into account. We remark that if the standing pulse converges to zero exponentially at onset (which is not generic but can be enforced by additional $\mathbb{Z}_{2}$-symmetries), the resulting bifurcation is easier to analyse (see, for instance, $[28,29]$ for existence results in this different context).

Oscillatory instabilities have served as our primary motivation for investigating absolute instabilities. We believe that Hopf bifurcations of standing pulses may be responsible for the creation of 1d-spirals such as the ones reported in the CIMA reaction by [13].

\subsection{Setup}

We consider reaction-diffusion systems

$$
u_{t}=D u_{x x}+f(u ; \mu), \quad x \in \mathbb{R}
$$

for $u \in \mathbb{R}^{n}$ and $\mu \in \mathbb{R}$, where $D=\operatorname{diag}\left(d_{j}\right)>0$ is a positive diagonal matrix and the nonlinearity $f: \mathbb{R}^{n} \times \mathbb{R} \rightarrow \mathbb{R}^{n}$ is smooth. We assume that $f(0 ; \mu)=0$ for all $\mu$.

Throughout this paper, we assume that (1.1) has a symmetric standing pulse $u(x, t)=q(x)$ for $\mu=0$ so that $q(x)=q(-x)$ for all $x$ and $q(x) \rightarrow 0$ as $|x| \rightarrow \infty$. In particular, symmetric standing pulses satisfy the steady-state equation

$$
D u_{x x}+f(u ; \mu)=0
$$


and lie in the fixed-point space of the $\mathbb{Z}_{2}$-symmetry given by $x \mapsto-x$ that is respected by (1.2). Alternatively, any standing pulse corresponds to a homoclinic orbit of the standing-wave ODE

$$
\begin{aligned}
& u^{\prime}=v \\
& v^{\prime}=-D^{-1} f(u ; \mu) .
\end{aligned}
$$

The $\mathbb{Z}_{2}$-symmetry of (1.2) translates into the following reversibility of the system (1.3) for $\mathbf{u}=$ $(u, v) \in \mathbb{R}^{2 n}$ : Reflecting

$$
\mathcal{R}: \quad(u, v) \longmapsto(u,-v)
$$

and reversing the spatial time $x \mapsto-x$ maps solutions into solutions. As a consequence, symmetric homoclinic orbits to hyperbolic equilibria can be found as intersections of the $n$-dimensional unstable manifold of the equilibrium with the $n$-dimensional fixed point subspace $\operatorname{Fix}(\mathcal{R})=\{(u, v) ; v=$ $0\}$. Whenever such an intersection is transverse, the homoclinic orbit persists as the parameter $\mu$ is varied. Note that (1.3) is, in general, not Hamiltonian unless $f$ is a gradient.

The linearization of (1.1) about the pulse $q(x)$ is given by the operator

$$
\mathcal{L}_{*}=D \partial_{x x}+f_{u}(q(x) ; 0)
$$

Since we are interested in its essential spectrum, we shall also linearize (1.1) about the trivial rest state $u=0$ which results in the operator

$$
\mathcal{L}_{\mu}=D \partial_{x x}+f_{u}(0 ; \mu)
$$

We consider the above operators on the space $C_{\text {unif }}^{0}\left(\mathbb{R}, \mathbb{C}^{n}\right)$ of bounded and uniformly continuous functions. Thus, we have

$$
\mathcal{L}_{*}, \mathcal{L}_{\mu}: \quad C_{\text {unif }}^{2}\left(\mathbb{R}, \mathbb{C}^{n}\right) \subset C_{\text {unif }}^{0}\left(\mathbb{R}, \mathbb{C}^{n}\right) \longrightarrow C_{\text {unif }}^{0}\left(\mathbb{R}, \mathbb{C}^{n}\right)
$$

We shall also use the subspaces $C_{\text {even }}^{0}\left(\mathbb{R}, \mathbb{C}^{n}\right)$ and $C_{\text {odd }}^{0}\left(\mathbb{R}, \mathbb{C}^{n}\right)$ of even and odd functions, respectively, in $C_{\text {unif }}^{0}\left(\mathbb{R}, \mathbb{C}^{n}\right)$.

We say that a complex number $\lambda$ is not in the essential spectrum of an operator $\mathcal{L}$ if $[\mathcal{L}-\lambda]$ is Fredholm with index zero. It is then true that the essential spectra of $\mathcal{L}_{*}$ and $\mathcal{L}_{0}$ coincide. Furthermore, Fourier transform shows that the essential spectrum of $\mathcal{L}_{\mu}$ is given by

$$
\operatorname{spec}\left(\mathcal{L}_{\mu}\right)=\{\lambda \in \mathbb{C} ; d(\lambda, \mathrm{i} k ; \mu)=0 \text { for some } k \in \mathbb{R}\}
$$

where the linear dispersion relation $d(\lambda, \nu ; \mu)$ is defined as

$$
d(\lambda, \nu ; \mu):=\operatorname{det}\left[\nu^{2} D+f_{u}(0 ; \mu)-\lambda\right], \quad(\lambda, \nu, \mu) \in \mathbb{C} \times \mathbb{C} \times \mathbb{R} .
$$

Note that the essential spectrum of $\mathcal{L}_{\mu}$ consists of curves of the form $\lambda=\lambda_{*}(k ; \mu)$ with $k \in \mathbb{R}$. These curves satisfy the equation $d(\lambda(k ; \mu), \mathrm{i} k ; \mu)=0$ for all $k \in \mathbb{R}$.

In the following sections, we summarize our hypotheses and results for each of the three scenarios mentioned in the preceding section. 


\subsection{Pitchfork bifurcations}

We begin with spatially-homogeneous instabilities where essential spectrum crosses the imaginary axis at $\lambda=0$ for $k=0$. We assume that this happens in a generic fashion as outlined in the following hypothesis.

Hypothesis 1 (Homogeneous instability) The essential spectrum of $\mathcal{L}_{\mu}$ close to the imaginary axis is given by a unique curve $\lambda(k ; \mu)$, defined for $k$ close to zero, that crosses the imaginary axis with nonzero speed as $\mu$ is increased through zero. More precisely, the dispersion relation $d(\lambda, \nu ; \mu)$ satisfies

$$
d(0,0 ; 0)=0, \quad d_{\lambda}(0,0 ; 0) \cdot d_{\nu \nu}(0,0 ; 0)<0, \quad d_{\lambda}(0,0 ; 0) \cdot d_{\mu}(0,0 ; 0)<0
$$

and that $d(\mathrm{i} \omega, \mathrm{i} k ; 0) \neq 0$ for $(\omega, k) \neq(0,0)$.

We now consider spatially-homogeneous solutions of (1.1) which satisfy the kinetic equation

$$
u_{t}=f(u ; \mu)
$$

In a neighborhood of $(u, \mu)=0$, the dynamics of this ODE can then be reduced to a one-dimensional center manifold with a vector field

$$
\dot{a}=g(a ; \mu), \quad a \in \mathbb{R} .
$$

We assume that the resulting bifurcation is a supercritical pitchfork bifurcation.

Hypothesis 2 (Supercritical pitchfork bifurcation) The reduced vector field $g(a ; \mu)$ on the one-dimensional center manifold is odd in a for all $\mu$ close to zero and $g_{a a a}(0 ; 0)<0$.

We denote by $u_{ \pm}(\mu)$ the two bifurcating equilibria of (1.4). Lastly, we require that the pulse $q(x)$ is non-degenerate.

Hypothesis 3 (Standing pulse) The standing pulse $q(x)$ is symmetric, the null space of $\mathcal{L}_{*}$ posed on $C_{\text {even }}^{0}\left(\mathbb{R}, \mathbb{C}^{n}\right)$ is trivial, and $|q(x)| \mathrm{e}^{\delta|x|} \rightarrow \infty$ as $|x| \rightarrow \infty$ for each $\delta>0$.

As already mentioned, if the standing pulse converges to zero exponentially at onset, the resulting bifurcation can be analysed without using blow-up techniques [28, 29].

Theorem 1 (Existence: Pitchfork) Assume that the Hypotheses 1, 2 and 3 are met. For all $\mu$ sufficiently small, there exists a symmetric standing pulse $q(x ; \mu)$ that depends continuously on $\mu$ as an element of $C_{\mathrm{unif}}^{0}\left(\mathbb{R}, \mathbb{R}^{n}\right)$. These pulses select one of the two bifurcating homogeneous equilibria $u_{+}(\mu)$ or $u_{-}(\mu)$ for $\mu>0$ and are asymptotic to $u=0$ for $\mu \leq 0$. Besides these pulses, there are no other standing pulses that are close in $C_{\text {unif }}^{0}\left(\mathbb{R}, \mathbb{R}^{n}\right)$ to the primary pulse $q(x)$. (See Figure 1$)$. 


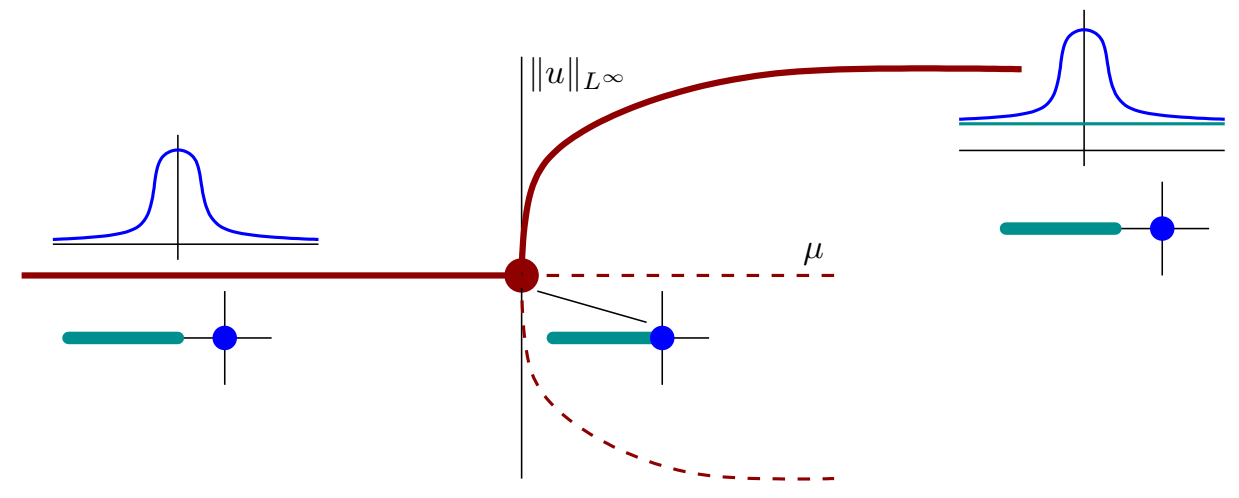

Figure 1: The bifurcation diagram for standing pulses to homogeneous states that undergo a supercritical pitchfork bifurcation. The insets illustrate the profiles of the standing pulses and their PDE spectra.

If the standing pulse is marginally stable at $\mu=0$, then the bifurcating pulses are asymptotically stable for $\mu \neq 0$.

Hypothesis 4 (Marginal stability) The eigenvalue $\lambda=0$ of $\mathcal{L}_{*}$ in $C_{\text {unif }}^{0}\left(\mathbb{R}, \mathbb{C}^{n}\right)$ has geometric and algebraic multiplicity equal to one, and the spectrum of $\mathcal{L}_{*}$ is contained in $\{\operatorname{Re} \lambda<0\} \cup\{0\}$.

Theorem 2 (Stability: Pitchfork) Assume that the Hypotheses 1, 2, 3 and 4 are met. The pulses $q(x ; \mu)$ that we found in Theorem 1 are asymptotically stable for $\mu \neq 0$. In particular, we can find a constant $\delta>0$ so that the spectrum of the linearization of $(1.1)$ about $q(x ; \mu)$ is contained in $\{\operatorname{Re} \lambda \leq-\delta \mu\}$ uniformly in $\mu \neq 0$ close to zero with the exception of a simple eigenvalue at $\lambda=0$ with eigenfunction $q_{x}(x ; \mu)$.

The above stability result can be formulated and proved in terms of an appropriate Evans function. Following [20], we set $\lambda=\gamma^{2}$ for $\gamma \approx 0$ and extend the Evans function $\check{\mathcal{E}}(\gamma)$ for $\mu=0$ as a $\mathcal{C}^{2}$ function into $\gamma=0$ on an appropriate Riemann surface for $\operatorname{Re} \gamma>0$. Hypothesis 4 implies that $\check{\mathcal{E}}^{\prime \prime}(0) \neq 0$. The above theorem is then proved by showing that the Evans function $\check{\mathcal{E}}$ is continuous in the parameter $\mu$, so that $\gamma=0$ is the unique zero of the Evans function for all $\mu$.

Remark 1.1 If the eigenvalue $\lambda=0$ of $\mathcal{L}_{*}$ in $C_{\text {unif }}^{0}\left(\mathbb{R}, \mathbb{C}^{n}\right)$ has geometric multiplicity one, then its algebraic multiplicity is one if, and only if, the Melnikov integral

$$
M:=\int_{-\infty}^{\infty}\left\langle q^{*}(x), q^{\prime}(x)\right\rangle \mathrm{d} x
$$

is nonzero, where $q^{*}(x)$ is the second component of the unique (up to scalar multiples) bounded, nonzero solution of the adjoint variational equation

$$
\left(\begin{array}{l}
u_{x} \\
v_{x}
\end{array}\right)=-\left(\begin{array}{cc}
0 & 1 \\
-D^{-1} f_{u}(q(x) ; 0) & 0
\end{array}\right)^{T}\left(\begin{array}{l}
u \\
v
\end{array}\right)
$$


While we will not give a proof of the above remark, we wish to mention that the results that we shall establish in Section 2.1 demonstrate that both $q(x)$ and $q^{*}(x)$ will decay algebraically to zero as $|x| \rightarrow \infty$. The connection between the Melnikov integral and the algebraic multiplicity of $\lambda=0$ can be proved using the results in Section 3.3.

\subsection{Turing instability}

Turing instabilities are characterized by the following assumption.

Hypothesis 5 (Turing instability) The essential spectrum of $\mathcal{L}_{\mu}$ close to the imaginary axis is given by precisely two curves $\lambda_{ \pm}(k ; \mu)$, defined for $k$ close to $\pm k_{*}$ for some $k_{*} \neq 0$, that cross the imaginary axis with nonzero speed as $\mu$ is increased through zero. More precisely, the dispersion relation $d(\lambda, \nu ; \mu)$ satisfies

$$
d\left(0, \mathrm{i} k_{*} ; 0\right)=0, \quad d_{\lambda}\left(0, \mathrm{i} k_{*} ; 0\right) \cdot d_{\nu \nu}\left(0, \mathrm{i} k_{*} ; 0\right)<0, \quad d_{\lambda}\left(0, \mathrm{i} k_{*} ; 0\right) \cdot d_{\mu}\left(0, \mathrm{i} k_{*} ; 0\right)<0
$$

and that $d(\mathrm{i} \omega, \mathrm{i} k ; 0) \neq 0$ for $(\omega, k) \neq\left(0, \pm k_{*}\right)$.

To discuss the resulting steady-state equation, we scale the spatial variable $x=k y$ and restrict ourselves to functions that are even and $2 \pi$-periodic in $y$. The equation for $u(y, t)$ is given by

$$
u_{t}=D k^{2} u_{y y}+f(u ; \mu)
$$

which has a one-dimensional center manifold near $u=0$ for $(\mu, k)$ close to $\left(0, k_{*}\right)$ with vector field

$$
\dot{a}=g(a ; k, \mu), \quad a \in \mathbb{R} .
$$

Hypothesis 6 (Supercritical Turing instability) The coefficient $g_{a a a}\left(0 ; k_{*}, 0\right)<0$ is negative.

Note that the vector field on the center manifold is always odd by equivariance of (1.6) with respect to the shift $x \mapsto x+\pi$. For each $k$ close to $k_{*}$ and each $\mu$ with

$$
\mu>\mu_{\mathrm{bif}}(k)=\frac{1}{2} \mu_{\mathrm{bif}}^{0} k^{2}+\mathrm{O}\left(k^{3}\right), \quad \mu_{\mathrm{bif}}^{0}:=-\frac{d_{\mu}\left(0, \mathrm{i} k_{*}, 0\right)}{d_{k k}\left(0, \mathrm{i} k_{*}, 0\right)},
$$

there exists a unique even spatially-periodic Turing pattern $u(x ; k, \mu)$. In fact [17], the patterns $u(x ; k, \mu)$ are spectrally stable inside the Eckhaus boundary $\mu>\mu_{\mathrm{eck}}(k)=\frac{3}{2} \mu_{\mathrm{bif}}^{0} k^{2}+\mathrm{O}\left(k^{3}\right)$.

Theorem 3 (Existence: Turing) Assume that the Hypotheses 3, 5 and 6 are met, then there are positive constants $\delta$ and $C$ so that the following is true. For each $k$ inside the Eckhaus-stable band sufficiently close to $k_{*}$, there is a $\mu^{*}>\mu_{\mathrm{eck}}(k)$ with the following properties. For $\mu_{\mathrm{eck}}(k)<\mu<\mu^{*}$, there exist a phase $\theta(k, \mu)$ and a symmetric standing pulse $q(x ; k, \mu)$ which depends continuously on $(k, \mu)$ in $C_{\mathrm{loc}}^{0}$ such that

$$
|q(x ; k, \mu)-u(x+\theta(k, \mu) ; k, \mu)| \leq C \mathrm{e}^{-\delta \mu|x|}
$$




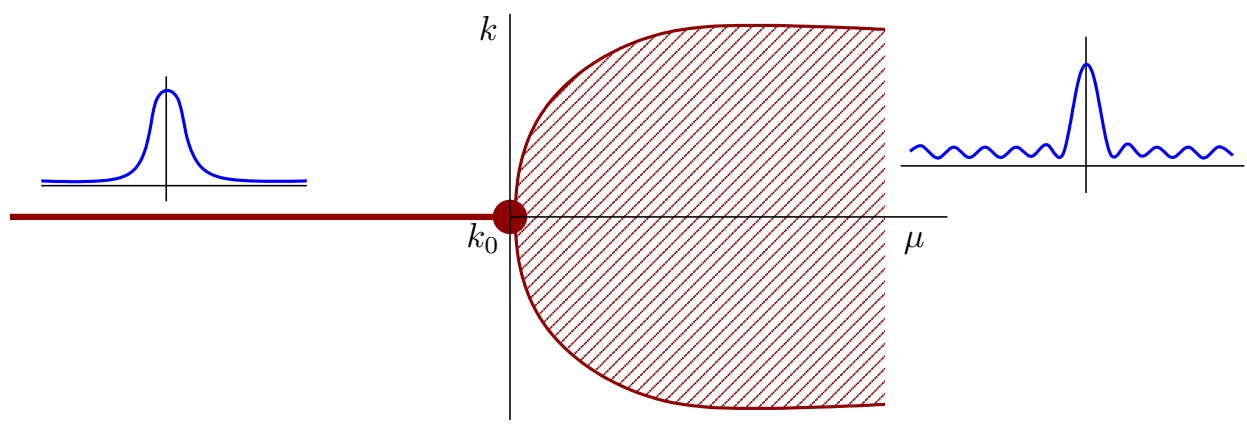

Figure 2: The bifurcation diagram for standing pulses to homogeneous states that undergo a supercritical Turing instability. The shaded region in $(\mu, k)$-space corresponds to the regime where Eckhaus-stable Turing patterns with wavenumber $k$ exist. The insets illustrate the profiles of the standing pulses.

for $x \in \mathbb{R}$. The pulse $q$ and its phase $\theta$ are unique in a neighborhood of the primary pulse $q(x)$ in $C_{\mathrm{loc}}^{0}$. For each $\mu<0$ sufficiently small, there exists a unique standing pulse, which is in fact symmetric and localized, in a neighborhood of the primary pulse $q(x)$, while localized pulses close to $q(x)$ do not exist for $\mu>0$. (See Figure 2).

Theorem 4 (Stability: Turing) Assume that the Hypotheses 3, 4, 5 and 6 are met. For $\mu>0$, the pulses $q(x ; k, \mu)$ that are described by Theorem 3 are spectrally stable: the spectrum of the linearization of (1.1) about $q(x ; k, \mu)$ is contained in $\{\operatorname{Re} \lambda<0\} \cup\{0\}$, and the algebraic multiplicity of $\lambda=0$ in $C_{\text {unif }}^{0}\left(\mathbb{R}, \mathbb{C}^{n}\right)$ is one.

For Turing bifurcations, spectral stability does not obviously imply asymptotic stability since $\lambda=0$ is always contained in the essential spectrum of the bifurcating pulses. The above theorem states that isolated unstable eigenvalues are not created in a generic Turing instability of standing pulses.

\subsection{Oscillatory instability}

Lastly, we consider oscillatory instabilities.

Hypothesis 7 (Homogeneous oscillatory instability) The essential spectrum of $\mathcal{L}_{\mu}$ near the imaginary axis is given by precisely two curves $\lambda_{ \pm}(k ; \mu)$, defined for $k$ close to zero, that cross the imaginary axis with nonzero speed at $\pm \mathrm{i} \omega_{*}$ for some $\omega_{*} \neq 0$ as $\mu$ is increased through zero. More precisely, the dispersion relation $d(\lambda, \nu ; \mu)$ satisfies

$$
d\left(\mathrm{i} \omega_{*}, 0 ; 0\right)=0, \quad d_{\lambda}\left(\mathrm{i} \omega_{*}, 0 ; 0\right) \cdot d_{\nu \nu}\left(\mathrm{i} \omega_{*}, 0 ; 0\right)<0, \quad d_{\lambda}\left(\mathrm{i} \omega_{*}, 0 ; 0\right) \cdot d_{\mu}\left(\mathrm{i} \omega_{*}, 0 ; 0\right)<0
$$

and $d(\mathrm{i} \omega, \mathrm{i} k ; 0) \neq 0$ for $(\omega, k) \neq\left( \pm \omega_{*}, 0\right)$.

As a consequence of Hypothesis 7, the kinetic equation

$$
u_{t}=f(u ; \mu)
$$


undergoes a Hopf bifurcation at $(u, \mu)=0$, and the dynamics of (1.7) near $u=0$ can be reduced to a two-dimensional center manifold.

Hypothesis 8 (Supercritical Hopf bifurcation) The cubic normal form of the reduced vector field on the center manifold of (1.7) at $\mu=0$ is given by

$$
A_{t}=\mathrm{i} \omega_{*} A-(1+\mathrm{i} \beta)|A|^{2} A
$$

for some $\beta \in \mathbb{R}$.

Upon scaling the spatial variable $x$, we may assume that $\partial_{k k} \lambda_{ \pm}(0 ; 0)=1+\mathrm{i} \alpha$. Since the coefficients $\alpha$ and $\beta$ will play an important role in the statement of our results, we remark that procedures for computing them for a given reaction-diffusion system can be found, for instance, in [12].

As proved in [26], the dynamics of the reaction-diffusion system close to the steady state $u=0$ in the supercritical regime $\mu>0$ is approximated by the complex Ginzburg-Landau equation (CGL)

$$
A_{T}=(1+\mathrm{i} \alpha) A_{X X}+A-(1+\mathrm{i} \beta)|A|^{2} A, \quad(X, T)=(\sqrt{\mu} x, \mu t) .
$$

Standing modulated waves of the CGL are of the form

$$
A(X, T)=\mathrm{e}^{-\mathrm{i} \Omega T} A(X)
$$

where $A(X)$ together with its derivative satisfies the ordinary differential equation (ODE)

$$
\begin{aligned}
& A_{X}=B \\
& B_{X}=\frac{1}{1+\mathrm{i} \alpha}\left[-(1+\mathrm{i} \Omega) A+(1+\mathrm{i} \beta)|A|^{2} A\right]
\end{aligned}
$$

which respects the reverser $\mathcal{R}_{\mathrm{cgl}}:(A, B) \mapsto(-A, B)$. The wave trains

$$
A_{\mathrm{w}}(X ; \kappa)=\sqrt{1-\kappa^{2}} \mathrm{e}^{\mathrm{i} \kappa X}
$$

are periodic orbits of (1.9) for

$$
\Omega=\Omega_{\mathrm{nl}}(\kappa):=\beta+(\alpha-\beta) \kappa^{2}
$$

In our analysis, we shall need certain transversality properties of the Nozaki-Bekki holes $A_{\mathrm{nb}}$ which are standing waves of the CGL $(1.8)$ that are spatially asymptotic to the wave train $A_{\mathrm{w}}(\cdot ; \kappa)$ for a selected wavenumber $\kappa$.

Hypothesis 9 (Nozaki-Bekki holes) There is a wavenumber $\kappa_{*}$ and a solution $A_{\mathrm{nb}}(X)$ of $(1.9)$ such that the following is true:

(i) The center-unstable manifold $W^{\mathrm{cu}}\left(A_{\mathrm{w}}\left(\cdot ; \kappa_{*}\right)\right)$ of the wave $A_{\mathrm{w}}\left(X ; \kappa_{*}\right)$ as a solution to (1.9) satisfies $\operatorname{dim} W^{\mathrm{cu}}\left(A_{\mathrm{w}}\left(\cdot ; \kappa_{*}\right)\right)=2$.

(ii) $\left(A_{\mathrm{nb}}, A_{\mathrm{nb}}^{\prime}\right)(0) \in \operatorname{Fix}\left(\mathcal{R}_{\mathrm{cgl}}\right) \cap W^{\mathrm{cu}}\left(A_{\mathrm{w}}\left(\cdot ; \kappa_{*}\right)\right)$. 
(iii) The above intersection is transverse at $\left(A_{\mathrm{nb}}, A_{\mathrm{nb}}^{\prime}\right)(0)$ when considered in the extended phase space $(A, B, \Omega)$.

The assumptions on the stability properties of the Nozaki-Bekki holes are summarized in the following hypothesis.

Hypothesis 10 (Nozaki-Bekki holes: Stability) (i) The selected wave train with wavenumber $\kappa_{*}$ is spectrally stable for (1.8).

(ii) The linearization of (1.8) about the Nozaki-Bekki hole $A_{\mathrm{nb}}$ considered in the space $C_{\text {odd }}^{0}(\mathbb{R}, \mathbb{C})$ has a simple eigenvalue at the origin and no other spectrum in the closed right-half plane.

There is strong numerical evidence that Hypotheses 9 and 10 are true in a large region in parameter space $(\alpha, \beta)$ (see $[1,11]$ for references). However, they have been verified analytically only in the limit to the real Ginzburg-Landau equation:

Proposition 1.2 There exists a constant $\delta>0$ so that Hypotheses 9 and 10 are true for all $(\alpha, \beta)$ with $|\alpha|,|\beta|<\delta$ and $0<|\alpha-\beta|<\delta$ in which case

$$
\kappa_{*}=\frac{\sqrt{2}\left(1+\alpha^{2}\right)}{3(1+\alpha \beta)}(\beta-\alpha)+\mathrm{O}\left(|\beta-\alpha|^{2}\right) .
$$

We remark that the existence part of this proposition is well known (see again $[1,11]$ for references). The stability of the Nozaki-Bekki holes has been investigated in [7]; however, the result stated in [7] is incorrect, and we shall give a corrected statement, and its proof, in Appendix A.

We now return to the reaction-diffusion system (1.1). The above assumptions imply that (1.1) has a family of wave trains $u_{\mathrm{w}}(k x-\omega t ; k, \mu)$ for each $k$ close to zero and $\mu>\mu_{\mathrm{bif}}(k)>0$ where $\omega:=\omega_{*}+\Omega_{\mathrm{nl}}(k / \sqrt{\mu}) \mu+\mathrm{O}\left(\mu^{3 / 2}\right)$ and $u_{\mathrm{w}}$ is $2 \pi$-periodic in its first argument.

Lastly, we assume that (1.1) admits a symmetric standing pulse for $\mu=0$.

Hypothesis 11 (Standing pulse) There exists a symmetric standing pulse $q(x)$ of (1.1) with $\mu=0$ such that the null space of $\mathcal{L}_{*}-\mathrm{i} \omega_{*} \ell$ is trivial in $C_{\mathrm{even}}^{0}\left(\mathbb{R}, \mathbb{C}^{n}\right)$ for all $\ell \in \mathbb{Z}$ and in $C_{\text {odd }}^{0}\left(\mathbb{R}, \mathbb{C}^{n}\right)$ for all odd $\ell \in \mathbb{Z}$.

Time-periodic pulses of the reaction-diffusion system can now be obtained by gluing together the standing pulse and the Nozaki-Bekki holes at $x= \pm \infty$.

Theorem 5 (Existence: Hopf) Assume that the Hypotheses 7, 8, 9 and 11 are met. For each $\mu>0$ sufficiently small, there exist symmetric and antisymmetric oscillating pulses, denoted by $q_{0}(x, t ; \mu)$ and $q_{\pi}(x, t ; \mu)$, respectively, with minimal temporal periods and frequencies

$$
T_{j}(\mu)=\frac{2 \pi}{\omega_{j}(\mu)}, \quad \omega_{j}(\mu)=\omega_{*}+\Omega_{\mathrm{nl}}\left(\kappa_{*}\right) \mu+\mathrm{O}\left(\mu^{2}\right), \quad j=0, \pi,
$$




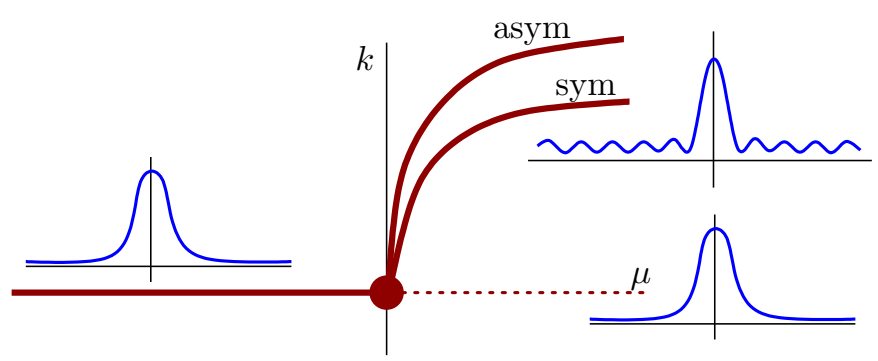

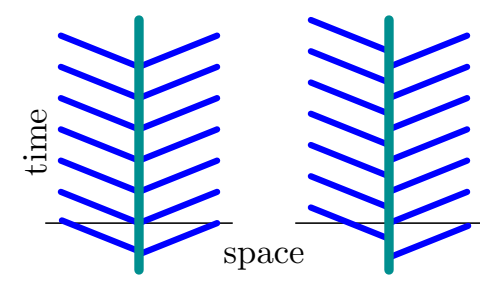

symmetric asymmetric

Figure 3: The bifurcation diagram for standing pulses to homogeneous states that undergo an oscillatory instability. The insets to the left illustrate snapshots in time of the profiles of the standing pulse for $\mu<0$ and of symmetric and asymmetric oscillatory pulses for $\mu>0$. The space-time plots to the right show the difference between the symmetric and the asymmetric modulated pulses.

that satisfy

$$
\begin{aligned}
& q_{0}(x, t ; \mu)=q_{0}\left(x, t+T_{0}(\mu) ; \mu\right)=q_{0}(-x, t ; \mu) \\
& q_{\pi}(x, t ; \mu)=q_{\pi}\left(x, t+T_{\pi}(\mu) ; \mu\right)=q_{\pi}\left(-x, t+\frac{T_{\pi}(\mu)}{2} ; \mu\right) .
\end{aligned}
$$

Both pulses depend continuously on $\mu$ in $C_{\mathrm{loc}}^{0}$, and they are asymptotic in space to the smallamplitude wave trains $u_{\mathrm{w}}$ with the selected wavenumber $k_{j}(\mu)=\sqrt{\mu} \kappa_{*}+\mathrm{O}(\mu)$ : There exist continuous functions $\theta_{j}(\mu) \in \mathbb{R}$ such that

$$
\left|q_{j}(x, t ; \mu)-u_{\mathrm{w}}\left(k_{j}(\mu) x-\omega_{j}(\mu) t+\theta_{j}(\mu) ; k_{j}(\mu), \mu\right)\right| \rightarrow 0
$$

as $x \rightarrow \infty$ uniformly in $t$ for $j=0, \pi$. (See Figure 3).

The theorem and its proof imply that both the phase and the group velocity of the asymptotic wave trains are directed away from the primary pulse. Thus, the bifurcating modulated pulses are sources in the terminology used in [21].

We remark that the primary localized pulse persists for $\mu>0$ (but is unstable). We also point out that the symmetric and antisymmetric pulses constructed in the theorem above are not unique. Many other modulated pulses can be found, but all of these involve sinks: One example are modulated pulses for which the group velocities of the asymptotic wave trains are directed towards the primary pulse [21]. Furthermore, modulated pulse may arise due to the interaction of several copies of the large sources that we constructed above with small-amplitude sinks that match the counter-propagating wave trains created by the sources.

Hypothesis 12 (Marginal stability) The spectrum of $\mathcal{L}_{*}$ is contained in $\{\operatorname{Re} \lambda<0\} \cup\left\{0, \pm \mathrm{i} \omega_{*}\right\}$. The eigenvalue $\lambda=0$ of $\mathcal{L}_{*}$ in $C_{\text {unif }}^{0}\left(\mathbb{R}, \mathbb{C}^{n}\right)$ has geometric and algebraic multiplicity equal to one, while the null space of $\mathcal{L}_{*} \pm \mathrm{i} \omega_{*}$ is trivial in $C^{0}\left(\mathbb{R}, \mathbb{C}^{n}\right)$.

The following stability result is formulated in terms of Evans functions. The interpretation for the spectrum is that any root of the Evans function on or to the right of the essential spectrum 

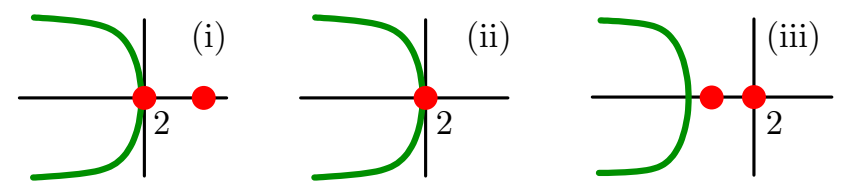

Figure 4: The spectra of the symmetric and antisymmetric defects are plotted for $\Lambda \neq 0$ : (i) unstable defect; (ii) stable defect; (iii) stable defect in a weighted $L^{2}$-space with norm $\|u\|^{2}:=$ $\int_{\mathbb{R}} \mathrm{e}^{-\eta|x|}|u(x)|^{2} \mathrm{~d} x$ for some small $\eta>0$ (which may depend on the bifurcation parameter $\mu$ ). The solid line represents the essential spectrum of the asymptotic wave trains. (See [21, Theorem 6.1] for details).

corresponds to a genuine Floquet exponent of the linearization of (1.1) about $q_{j}$, while roots to the left of the essential spectrum correspond to resonance poles (i.e. to eigenvalues in an exponentially weighted function space).

Theorem 6 (Stability: Hopf) Assume that the Hypotheses 7, 8, 9, 10, 11 and 12 are met. For $\mu>0$, the essential Floquet spectrum of the linearization of (1.1) about $q_{j}(x, t ; \mu)$ with $j=0, \pi$ is contained in $\{\operatorname{Re} \lambda<0\} \cup\{0\}$, and $\lambda=0$ is a Floquet exponent of geometric and algebraic multiplicity two. Furthermore, there are constants $\delta>0$ and $\Lambda \in \mathbb{R}$ such that the Evans function associated with $q_{j}$ has precisely one additional root $\lambda_{j}(\mu)$ in the region $\operatorname{Re} \lambda>-\delta \mu$ with the expansions

$$
\lambda_{0}(\mu)=\Lambda \mu^{3 / 2}+\mathrm{O}\left(\mu^{2}\right), \quad \lambda_{\pi}(\mu)=-\Lambda \mu^{3 / 2}+\mathrm{O}\left(\mu^{2}\right) .
$$

An expression for $\Lambda$ is given in (4.33). Generically, $\Lambda \neq 0$ so that either $q_{0}$ or $q_{\pi}$ is stable, while the other pulse has a single unstable Floquet exponent. (See Figure 4).

\subsection{Further remarks and outline of the paper}

The following result shows that the hypotheses of our main theorems are compatible.

Theorem 7 There exist three open, non-empty classes of one-parameter families of reactiondiffusion systems so that the equations in these three classes satisfy

- Hypotheses 1, 2, 3 and 4 [Pitchfork instability],

- Hypotheses 3, 4, 5 and 6 [Turing instability],

- Hypotheses 7, 8, 9, 10, 11 and 12 [Oscillatory instability],

respectively.

The plan of this paper is as follows. We prove the existence results in Section 2 and the stability results in Sections 3 and 4. After discussing Theorem 7 in Section 5, we conclude with a brief discussion in Section 6. Lastly, Proposition 1.2 is proved in Appendix A. 


\section{Existence}

\subsection{Pitchfork bifurcations: Existence}

We shall prove Theorem 1: We seek symmetric standing pulses of (1.1) as reversible homoclinic orbits of the standing-wave ODE

$$
\begin{aligned}
& u^{\prime}=v \\
& v^{\prime}=-D^{-1} f(u ; \mu)
\end{aligned}
$$

where $(u, v) \in \mathbb{R}^{2 n}$ and $\mu \in \mathbb{R}$. Hypotheses 1 and 2 on the linear dispersion relation and the nonlinearity, respectively, imply that the equilibrium $(u, v)=0$ undergoes a reversible pitchfork bifurcation at $\mu=0$. In particular, the center eigenspace of the linearization of (2.1) about the origin is two-dimensional, and we can therefore reduce the dynamics near the origin to a twodimensional, locally invariant center manifold for $\mu$ close to zero. Using $[27,(5.7)$ in $\S 2.5]$ and reversibility, we see that, possibly after transforming into appropriate normal-form coordinates, the vector field on the center manifold is given by

$$
\begin{aligned}
& A_{x}=B \\
& B_{x}=-\mu A+A^{3}+A \mathrm{O}\left(|A|^{4}+|B|^{4}+|\mu||A|^{2}+\mu^{2}|A|\right),
\end{aligned}
$$

and the reverser $\mathcal{R}$ is represented by the reflection $B \mapsto-B$. We refer to Figure 5 for an illustration of the expected phase diagram which will be confirmed by the forthcoming analysis.

For $\mu<0$, the origin of (2.2) has a one-dimensional unstable manifold. To determine the fate of the homoclinic orbit upon increasing $\mu$ through zero, we will need to continue the unstable manifold through $\mu=0$. Since the origin is not hyperbolic for $\mu=0$, we use geometric blow-up to isolate the unstable set of the origin. Background material on blow-up techniques can be found, for instance, in the review article [4]. We transform (2.2) with coordinates $(A, B, \mu) \in \mathbb{R}^{3}$ into polar coordinates that live on $\mathbb{R}_{+} \times S^{2}$ with the radius given by $R=A^{4}+B^{2}+\mu^{2}$. We use different charts to parametrize the sphere $S^{2}$.
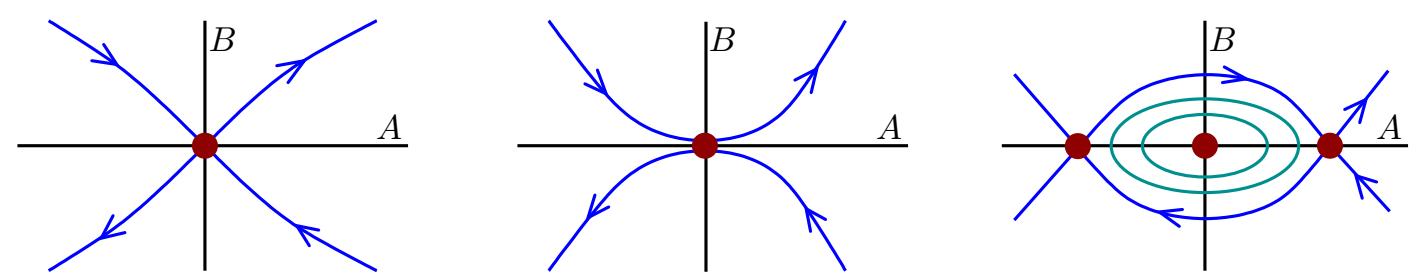

Figure 5: The phase diagrams of a supercritical pitchfork bifurcation for $\mu<0, \mu=0$ and $\mu>0$ (from left to right). 


\section{The singular chart}

We begin with the $A$-directional blow-up chart defined by

$$
A_{1}=A, \quad B_{1}=\frac{B}{A^{2}}, \quad \mu_{1}=\frac{\mu}{A^{2}},
$$

which contains the dynamics in the region $A \neq 0$ that we are interested in. In these coordinates, equation (2.2) becomes

$$
\begin{aligned}
A_{1}^{\prime} & =A_{1}\left[A_{1} B_{1}\right] \\
B_{1}^{\prime} & =A_{1}\left[1-\mu_{1}-2 B_{1}^{2}+\mathrm{O}\left(A_{1}\right)\right] \\
\mu_{1}^{\prime} & =A_{1}\left[-2 \mu_{1} B_{1}\right]
\end{aligned}
$$

where $^{\prime}=\mathrm{d} / \mathrm{d} x$. If we introduce the new evolution variable $y$ via $\mathrm{d} y / \mathrm{d} x=A_{1}(x)$ and set ${ }^{\cdot}=\mathrm{d} / \mathrm{d} y$, then (2.3) with $A_{1}>0$ becomes

$$
\begin{aligned}
\dot{A}_{1} & =A_{1} B_{1} \\
\dot{B}_{1} & =1-\mu_{1}-2 B_{1}^{2}+\mathrm{O}\left(A_{1}\right) \\
\dot{\mu}_{1} & =-2 \mu_{1} B_{1} .
\end{aligned}
$$

Note that flow lines are reversed for $A_{1}<0$.

We shall now analyse the flow of (2.4) and confirm that Figure 6 is an accurate description of the dynamics. First, note that the origin $(A, B)=0$ becomes the singular plane $\left\{A_{1}=0\right\}$ which is invariant under the flow. In addition, the $B_{1}$-axis, which corresponds to the origin $(A, B)=0$ at $\mu=0$, is invariant.

Checking equation (2.4) for equilibria, we find the two singular equilibria

$$
p_{\mathrm{s}}^{ \pm}=\left(A_{1}, B_{1}, \mu_{1}\right)=\left(0, \frac{ \pm 1}{\sqrt{2}}, 0\right)
$$

on the $B_{1}$-axis and the one-parameter family

$$
p_{\mathrm{w}}=p_{\mathrm{w}}\left(A_{1}\right)=\left(A_{1}, B_{1}, \mu_{1}\right)=\left(A_{1}, 0,1+\mathrm{O}\left(A_{1}\right)\right)
$$

of wave equilibria which correspond to the equilibria with $A_{1}=A>0$ that bifurcate in the pitchfork bifurcation.

The linearization of (2.4) about the singular equilibria $p_{\mathrm{s}}^{ \pm}$is given by

$$
\left(\begin{array}{ccc} 
\pm \frac{1}{\sqrt{2}} & 0 & \\
\mathrm{O}(1) & \mp \frac{4}{\sqrt{2}} & 0 \\
0 & 0 & \mp \frac{2}{\sqrt{2}}
\end{array}\right)
$$

where the $\mathrm{O}(1)$-term comes from differentiating the $\mathrm{O}\left(A_{1}\right)$-term in (2.4). If we restrict ourselves to the invariant plane $\mu_{1}=0$, which corresponds to $\mu=0$, then we see that the unstable set 

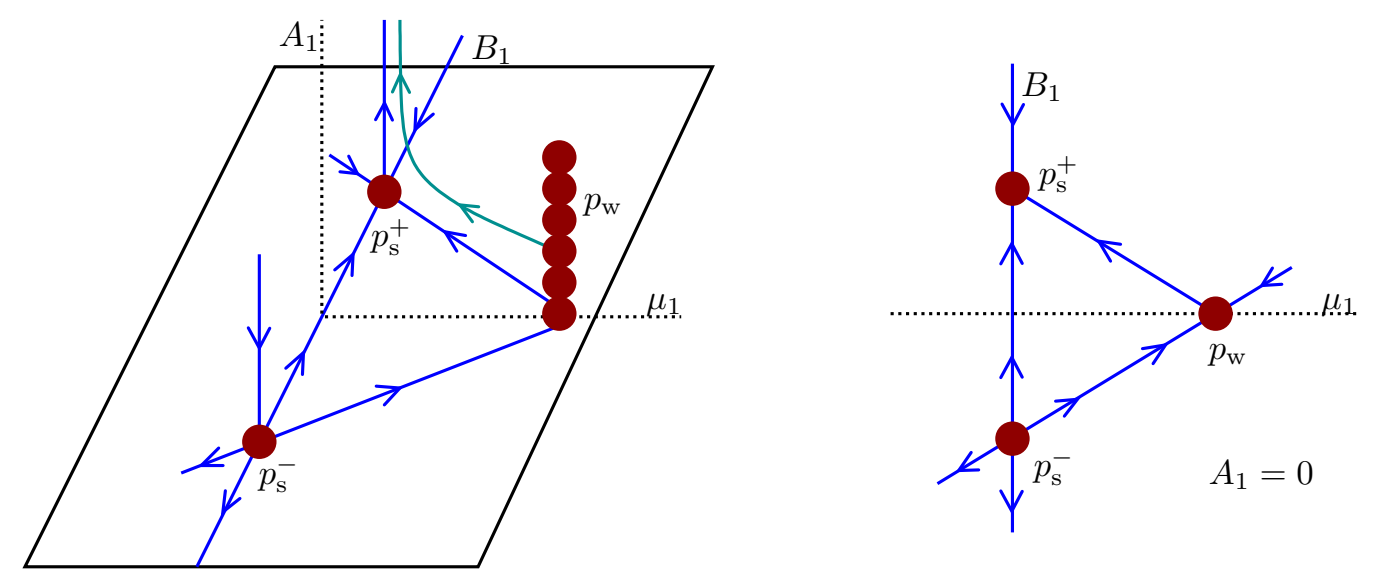

Figure 6: The dynamics of (2.4) and (2.6) are shown in the left and right figure, respectively. The dotted lines indicate coordinate axes. Each of the wave equilibria $p_{\mathrm{w}}$ has one-dimensional stable and unstable manifolds.

of the $B_{1}$-axis (i.e. the origin in $(A, B, \mu)$-coordinates) in the region $A_{1}>0$ is given by the onedimensional unstable manifold of the singular equilibrium $p_{\mathrm{s}}^{+}$(see Figure 6 ). This proves that the unstable set of the origin inside the center manifold is, for $\mu=0$, the union of two smooth manifolds $W^{\mathrm{u}}=W_{+}^{\mathrm{u}} \cup\{0\} \cup W_{-}^{\mathrm{u}}$.

Next, we analyse the family of wave equilibria $p_{\mathrm{w}}$. Within the invariant plane $\left\{A_{1}=0\right\},(2.4)$ reduces to

$$
\begin{aligned}
& \dot{B}_{1}=1-\mu_{1}-2 B_{1}^{2} \\
& \dot{\mu}_{1}=-2 \mu_{1} B_{1} .
\end{aligned}
$$

The wave equilibrium $p_{\mathrm{w}}(0)$ is a saddle for (2.6) and, as indicated in Figure 6, the heteroclinic orbit

$$
\left(B_{1}, \mu_{1}\right)=\left(\frac{\sqrt{2}}{4}\left(1+\tanh \left(\frac{x}{\sqrt{2}}\right)\right), \frac{1}{2}\left(1-\tanh \left(\frac{x}{\sqrt{2}}\right)\right)\right)
$$

connects $p_{\mathrm{w}}(0)$ to the $\operatorname{sink} p_{\mathrm{s}}^{+}$along the invariant line $B_{1}=\left(1-\mu_{1}\right) / \sqrt{2}$.

In summary, we have established that the flow of (2.4) is indeed as shown in Figure 6. Since we are interested in constructing homoclinic orbits to the wave equilibria $p_{\mathrm{w}}$, we need to understand the structure of the one-dimensional unstable manifolds of each wave equilibrium.

As indicated in Figure 6, the unstable manifolds for $A_{1}$ close to zero will follow the unstable manifold belonging to $A_{1}=0$ until we are close to the singular equilibrium $p_{\mathrm{s}}^{+}$. From there, we expect that the unstable manifolds for $A_{1} \neq 0$ will follow the one-dimensional unstable manifold of $p_{\mathrm{s}}^{+}$which points into the $A_{1}$-direction (see again Figure 6 ). In other words, we see that the unstable manifolds of the wave equilibria for $A_{1} \neq 0$ can be thought of as being constructed in a heteroclinic bifurcation where the unstable manifold of the wave equilibrium with $A_{1} \neq 0$ and the unstable manifold of the singular equilibrium $p_{\mathrm{s}}^{+}$are glued together. This will be a recurrent theme throughout this paper. 
We shall now make these comments precise and prove that the unstable manifold of each wave equilibrium can be continued smoothly to a fixed finite value of $A_{1}=A$, so that its limit, as the $A_{1}$-coordinate of $p_{\mathrm{w}}$ goes to zero, coincides with the unstable manifold of the singular equilibrium

$p_{\mathrm{s}}^{+}$. To prove this claim, we have to track the unstable manifold of each wave equilibrium as it passes near the singular equilibrium $p_{\mathrm{s}}^{+}$and derive expressions for its position relative to the unstable manifold of $p_{\mathrm{s}}^{+}$when evaluated at a finite fixed distance $A_{1}=\delta>0$ from $A_{1}=0$. First, note that the unstable manifold of the wave equilibrium $p_{\mathrm{w}}$ with $A_{1}=\varepsilon>0$ enters, after a finite time, a small fixed neighborhood of the singular equilibrium $p_{\mathrm{s}}^{+}$at distance $\mathrm{O}(\varepsilon)$ from the invariant plane $A_{1}=0$. To compute the location of the unstable manifold for a finite value $A_{1}=\delta$, we therefore have to analyse the transition map near the hyperbolic equilibrium $p_{\mathrm{s}}^{+}$. The analysis presented in Appendix B, which is based on the fact that the modulus of the stable eigenvalues of the linearization (2.5) about the singular equilibrium $p_{\mathrm{s}}^{+}$is twice that of the unstable eigenvalue, shows that the unstable manifold of the wave equilibrium with amplitude $A_{1}=\varepsilon$ is $\mathrm{O}\left(\varepsilon^{2}\right)$-close to the unstable manifold of the singular equilibrium $p_{\mathrm{s}}^{+}$within the plane given by $A_{1}=\delta$. Since we have $\mu_{1}=1$ close to each wave equilibrium, and therefore $\mu \approx A_{1}^{2}$, we conclude that the unstable manifold of the wave equilibrium is $\mathrm{O}(\mu)$-close to the singular unstable manifold. This shows that, outside a small fixed neighborhood of the origin, $W_{+}^{\mathrm{u}}$ can be continued in a continuous fashion as a smooth manifold to positive parameter values $\mu>0$.

\section{The rescaling chart}

Next, to show that we can extend the unstable manifold also to negative values $\mu<0$, we investigate the rescaling chart with coordinates

$$
A_{3}=\frac{A}{\sqrt{|\mu|}}, \quad B_{3}=\frac{B}{|\mu|}, \quad \mu_{3}=|\mu|,
$$

which, for $\mu<0$, results in the equation

$$
\begin{aligned}
& \dot{A}_{3}=B_{3} \\
& \dot{B}_{3}=A_{3}+A_{3}^{3}+\mathrm{O}\left(\sqrt{\left|\mu_{3}\right|}\right) \\
& \dot{\mu}_{3}=0 .
\end{aligned}
$$

Note that the coordinates in singular and rescaling charts are related via

$$
A_{1}=A_{3} \sqrt{\mu_{3}}, \quad B_{1}=\frac{B_{3}}{A_{3}^{2}}, \quad \mu_{1}=-\frac{1}{A_{3}^{2}} .
$$

For $\mu_{3}=0$, the unstable manifold of the saddle equilibrium $\left(A_{3}, B_{3}, \mu_{3}\right)=0$ can be evaluated at some distance from the origin so that its coordinates are $\left(A_{3}, B_{3}, \mu_{3}\right)=\left(a_{*}, b_{*}, 0\right)$ for some $a_{*}, b_{*}>0$. Using (2.9), the corresponding coordinates in the $A$-directional chart are given by $\left(A_{1}, B_{1}, \mu_{1}\right)=\left(0, b_{*} / a_{*}^{2},-1 / a_{*}^{2}\right)$. Thus, examining the flow of $(2.6)$, we see that the unstable manifold of $\left(A_{3}, B_{3}, \mu_{3}\right)=0$ connects to the stable singular equilibrium $p_{\mathrm{s}}^{+}$in the $A$-directional chart. The equilibrium $\left(A_{3}, B_{3}, \mu_{3}\right)=0$ continues as a family $\left(A_{3}, B_{3}, \mu_{3}\right)=\left(0,0, \mu_{3}\right)$ for all 
$\mu_{3}>0$. Using the same arguments as for the wave equilibria, we see that the unstable manifold of $\left(A_{3}, B_{3}, \mu_{3}\right)=\left(0,0, \mu_{3}\right)$ is $\mathrm{O}(\mu)$-close to the unstable manifold of the singular equilibrium $p_{\mathrm{s}}^{+}$when evaluated at a fixed finite value $\delta>0$ of $A_{1}$. Thus, we have shown that the unstable manifold can be continued as a smooth manifold in a continuous fashion to $\mu=0$ outside of a fixed small neighborhood of the origin $(A, B)=0$.

\section{Constructing the bifurcating pulse}

We have proved that, when measured at a fixed finite distance away from the origin, the unstable set of the origin for $\mu \leq 0$ and the unstable manifold of the bifurcating equilibrium for $\mu>0$ fit together inside the center manifold in a continuous fashion at $\mu=0$.

We shall now interpret these results in the entire phase space $\mathbb{R}^{2 n}$. For $\mu=0$, the center-unstable manifold of the origin contains the entire unstable set. Furthermore, the center-unstable manifold is fibered smoothly over the center manifold. Thus, the unstable set of the origin is the union $\mathcal{W}^{\mathrm{u}}(0)=$ $\mathcal{W}_{+}^{\mathrm{u}} \cup \mathcal{W}^{\mathrm{uu}} \cup \mathcal{W}_{-}^{\mathrm{u}}$ of three disjoint sets that are obtained from the corresponding decomposition $W^{\mathrm{u}}=W_{+}^{\mathrm{u}} \cup\{0\} \cup W_{-}^{\mathrm{u}}$ inside the center manifold by adding the strong unstable fibers. Note that we can extend all these manifolds in a continuous fashion to arbitrary $\mu$ close to zero by considering the unstable manifold of the origin for $\mu<0$ and of the small bifurcating wave equilibria for $\mu>0$.

By assumption, the standing pulse $q(x)$ is symmetric in $x$ at $\mu=0$ and therefore given as the intersection of $\mathcal{W}^{\mathrm{u}}$ with $\operatorname{Fix}(\mathcal{R})$. We required in Hypothesis 3 that $q(x) \mathrm{e}^{-\delta x} \rightarrow \infty$ as $x \rightarrow-\infty$ for each $\delta>0$ from which we infer that $\operatorname{Fix}(\mathcal{R}) \cap \mathcal{W}^{\text {uu }}=\emptyset$. Without loss of generality, we may therefore assume that $\mathcal{W}_{+}^{\mathrm{u}} \cap \operatorname{Fix}(\mathcal{R}) \neq \emptyset$. We claim that this intersection is transverse. If true, this implies the existence of intersections for any $\mu$ close to zero, and therefore Theorem 1 . It remains therefore to prove the claim.

We argue by contradiction and assume that the intersection is not transverse for $\mu=0$. As a consequence, since $\operatorname{Fix}(\mathcal{R})=\{v=0\}$, there exists then a nonzero vector $\left(u_{0}, 0\right)$ in the tangent space of $\mathcal{W}_{+}^{\mathrm{u}}$ at the point $(q(0), 0) \in \operatorname{Fix}(\mathcal{R})$. We consider the corresponding even nonzero solution of the linearized equation

$$
\left(\begin{array}{c}
u_{x} \\
v_{x}
\end{array}\right)=\left(\begin{array}{c}
v \\
-D^{-1} f_{u}(q(x) ; 0) u
\end{array}\right), \quad\left(\begin{array}{c}
u(0) \\
v(0)
\end{array}\right)=\left(\begin{array}{c}
u_{0} \\
0
\end{array}\right) .
$$

Since the tangent space of $\mathcal{W}_{+}^{\mathrm{u}}$ along the homoclinic orbit is the sum of the tangent space of $W_{+}^{\mathrm{u}}$ and to the strong unstable fiber, the solution to (2.10) is a linear combination of the derivative $\left(q^{\prime}(x), q^{\prime \prime}(x)\right)$ and an exponentially decaying solution that lies in the tangent spaces of the strong unstable fibers. Since $B_{1}=1 / \sqrt{2}$ for $A_{1}=0$, it is easy to show that $q(x)=\sqrt{2} / x+\mathrm{O}\left(1 / x^{2}\right)$ so that $q^{\prime} \in L^{2}$. Thus, the first component of the solution to (2.10) supplies a nonzero element of the null space of the linearized operator $\mathcal{L}_{*}$ on $L_{\text {even }}^{2}\left(\mathbb{R}, \mathbb{C}^{n}\right)$, and therefore also in $C_{\text {even }}^{0}\left(\mathbb{R}, \mathbb{C}^{n}\right)$, which contradicts Hypothesis 3. This completes the proof of Theorem 1.

We remark that a similar blow-up can be carried out for the adjoint variational equation that appears in Remark 1.1. Combining the resulting estimate with the one for $q_{x}(x)$ obtained above shows that the Melnikov integral in (1.5) converges. 


\section{$2.2 \quad$ Turing instabilities: Existence}

For Turing instabilities, we shall follow closely the strategy that we employed in the previous section to handle pitchfork bifurcations. A Turing instability that meets Hypotheses 5 and 6 causes a reversible 1:1-resonance at the equilibrium $(u, v)=0$. In particular, the center eigenspace is four-dimensional. We focus on the construction and continuation of the two-dimensional unstable manifold through the bifurcation point to $\mu>0$ within the four-dimensional center manifold. Once this is achieved, the arguments given in Section 2.1 apply verbatim, thus completing the proof by showing transversality of the relevant unstable manifold and the fixed-point space of $\mathcal{R}$ in the full phase space $\mathbb{R}^{2 n}$.

Hence, we restrict our attention to the four-dimensional center manifold. As shown in [5] (see also [25, Proposition 2.18]), appropriate transformations of the parameter $\mu$, suitable $\mu$-dependent time rescalings, normal-form transformations up to cubic order and appropriate scalings of the variables $(A, B) \in \mathbb{C}^{2}$ on the center manifold can be utilized to bring the reduced vector field into the form

$$
\begin{aligned}
& A_{x}=\mathrm{i} A+B+\mathrm{i} A P\left(|A|^{2}, \mathrm{i}[A \bar{B}-\bar{A} B] ; \mu\right) \\
& B_{x}=-\mu A+\mathrm{i} B+A|A|^{2}+\mathrm{i} B P\left(|A|^{2}, \mathrm{i}[A \bar{B}-\bar{A} B] ; \mu\right)+A Q\left(|A|^{2}, \mathrm{i}[A \bar{B}-\bar{A} B] ; \mu\right)+R(A, B ; \mu) .
\end{aligned}
$$

Here, $P(I, J ; \mu)$ and $Q(I, J ; \mu)$ are real polynomials in their real arguments $(I, J)$ with coefficients that depend smoothly on $\mu$. We also have $P(0,0 ; 0)=0, Q(0,0 ; 0)=\partial_{I} Q(0,0 ; 0)=\partial_{\mu} Q(0,0 ; 0)=0$, and the remainder term $R$ satisfies $R=\mathrm{O}\left(|A|^{2 m+3}+|B|^{2 m+3}\right)$ where $m$ is an arbitrarily large, but fixed, natural number.

We focus on the construction and continuation of the unstable manifold for $\mu \geq 0$ since the case $\mu<0$ is completely analogous to the construction in the preceding section. As before, we use the coordinates

$$
A_{1}=\frac{A}{|A|} \in S^{1}, \quad B_{1}=\frac{B}{|A|^{2}} \in \mathbb{C}, \quad \mu_{1}=\frac{\mu}{|A|^{2}} \in \mathbb{R}
$$

in the $A$-directional blow-up chart and introduce the complex variable

$$
N=A_{1} \bar{B}_{1}=N_{\mathrm{r}}+\mathrm{i} N_{\mathrm{i}}, \quad N_{\mathrm{r}}, N_{\mathrm{i}} \in \mathbb{C} .
$$

Equation (2.11) becomes

$$
\begin{aligned}
A_{1}^{\prime}= & \mathrm{i} A_{1}+|A|\left[B_{1}-A_{1} N_{\mathrm{r}}\right]+\mathrm{i} A_{1}|A|^{2} \check{P}\left(|A|^{2}, N_{\mathrm{i}} ; \mu_{1}\right) \\
B_{1}^{\prime}= & \mathrm{i} B_{1}+|A|\left[\left(1-\mu_{1}\right) A_{1}-2 B_{1} N_{\mathrm{r}}\right]+\mathrm{i} B_{1}|A|^{2} \check{P}\left(|A|^{2}, N_{\mathrm{i}} ; \mu_{1}\right)+A_{1}|A|^{2} \check{Q}\left(|A|^{2}, N_{\mathrm{i}} ; \mu_{1}\right) \\
& +|A|^{2 m+1} \check{R}\left(|A|, A_{1}, B_{1} ; \mu_{1}\right) \\
\mu_{1}^{\prime}= & |A|\left[-2 \mu_{1} N_{\mathrm{r}}\right] \\
|A|^{\prime}= & |A|^{2} N_{\mathrm{r}}
\end{aligned}
$$

where $^{\prime}=\mathrm{d} / \mathrm{d} x$. Note that $\check{P}, \check{Q}, \check{R}$ are smooth but do not necessarily vanish at $\left(A_{1}, B_{1}, \mu_{1},|A|\right)=0$; with a slight abuse of notation, we shall omit their dependence on $\mu_{1}$ from now on. The normal-form part of (2.12), i.e. the equation without the remainder term $\check{R}$, respects the continuous symmetry

$$
\left(A_{1}, B_{1}\right) \longmapsto \mathrm{e}^{\mathrm{i} \phi}\left(A_{1}, B_{1}\right), \quad \phi \in \mathbb{R}
$$


which is generated by the evolution of the linear part of the equation. To exploit this symmetry and eliminate the linear part, we introduce the new coordinates

$$
a_{1}=A_{1} \mathrm{e}^{-\mathrm{i} x} \in S^{1}, \quad b_{1}=B_{1} \mathrm{e}^{-\mathrm{i} x} \in \mathbb{C}
$$

so that (2.12) becomes

$$
\begin{aligned}
a_{1}^{\prime}= & |A|\left[b_{1}-a_{1} n_{\mathrm{r}}\right]+\mathrm{i} a_{1}|A|^{2} \check{P}\left(|A|^{2}, n_{\mathrm{i}}\right) \\
b_{1}^{\prime}= & |A|\left[\left(1-\mu_{1}\right) a_{1}-2 b_{1} n_{\mathrm{r}}\right]+\mathrm{i} b_{1}|A|^{2} \check{P}\left(|A|^{2}, n_{\mathrm{i}}\right)+a_{1}|A|^{2} \check{Q}\left(|A|^{2}, n_{\mathrm{i}}\right) \\
& +|A|^{2 m+1} \check{R}\left(|A|, a_{1} \mathrm{e}^{\mathrm{i} x}, b_{1} \mathrm{e}^{\mathrm{i} x}\right) \\
\mu_{1}^{\prime}= & |A|\left[-2 \mu_{1} n_{\mathrm{r}}\right] \\
|A|^{\prime}= & |A|^{2} n_{\mathrm{r}}
\end{aligned}
$$

where $n=a_{1} \bar{b}_{1}=A_{1} \bar{B}_{1}=N$. We eliminate the Euler multiplier $|A|$ by replacing the independent variable $x$ by the new independent variable $y$ via $\mathrm{d} y / \mathrm{d} x=|A|$ so that $x=\varphi(y)$ and $\mathrm{d} \varphi / \mathrm{d} y=1 /|A|$. We obtain

$$
\begin{aligned}
\dot{a}_{1} & =b_{1}-a_{1} n_{\mathrm{r}}+\mathrm{i} a_{1}|A| \check{P}\left(|A|^{2}, n_{\mathrm{i}}\right) \\
\dot{b}_{1} & =\left(1-\mu_{1}\right) a_{1}-2 b_{1} n_{\mathrm{r}}+\mathrm{i} b_{1}|A| \check{P}\left(|A|^{2}, n_{\mathrm{i}}\right)+a_{1}|A| \check{Q}\left(|A|^{2}, n_{\mathrm{i}}\right)+|A|^{2 m} \check{R}\left(|A|, a_{1} \mathrm{e}^{\mathrm{i} \varphi}, b_{1} \mathrm{e}^{\mathrm{i} \varphi}\right) \\
\dot{\mu}_{1} & =-2 \mu_{1} n_{\mathrm{r}} \\
|\dot{A}| & =|A| n_{\mathrm{r}} \\
\dot{\varphi} & =|A|^{-1}
\end{aligned}
$$

where ${ }^{\cdot}=\mathrm{d} / \mathrm{d} y$. If we further substitute $\psi=|A|^{2} \varphi$, we get

$$
\begin{aligned}
\dot{a}_{1}= & b_{1}-a_{1} n_{\mathrm{r}}+\mathrm{i} a_{1}|A| \check{P}\left(|A|^{2}, n_{\mathrm{i}}\right) \\
\dot{b}_{1}= & \left(1-\mu_{1}\right) a_{1}-2 b_{1} n_{\mathrm{r}}+\mathrm{i} b_{1}|A| \check{P}\left(|A|^{2}, n_{\mathrm{i}}\right)+a_{1}|A| \check{Q}\left(|A|^{2}, n_{\mathrm{i}}\right) \\
& +|A|^{2 m} \check{R}\left(|A|, a_{1} \mathrm{e}^{\mathrm{i} \psi /|A|^{2}}, b_{1} \mathrm{e}^{\mathrm{i} \psi /|A|^{2}}\right) \\
\dot{\mu}_{1}= & -2 \mu_{1} n_{\mathrm{r}} \\
|\dot{A}|= & |A| n_{\mathrm{r}} \\
\dot{\psi}= & |A|+2 \psi n_{\mathrm{r}} .
\end{aligned}
$$

We remark that the right-hand side is $C^{m-1}$ in its arguments and all derivatives are bounded up to and including the point $|A|=0$. The transformation back to the original variables is regular at all points for which $|A|=\delta>0$.

We begin by investigating the flow associated with (2.15) inside the invariant singular sphere $|A|=0$ with $\psi=0$. It is convenient to introduce the invariant polynomial $n=a_{1} \bar{b}_{1}=n_{\mathrm{r}}+\mathrm{i} n_{\mathrm{i}}$ associated with the symmetry (2.13) as a new variable which satisfies

$$
\begin{aligned}
& \dot{n}_{\mathrm{r}}=1-\mu_{1}+n_{\mathrm{i}}^{2}-2 n_{\mathrm{r}}^{2} \\
& \dot{n}_{\mathrm{i}}=-3 n_{\mathrm{i}} n_{\mathrm{r}} \\
& \dot{\mu}_{1}=-2 \mu_{1} n_{\mathrm{r}} .
\end{aligned}
$$


Note that the plane $n_{\mathrm{i}}=0$ is invariant under (2.16): The vector field on this plane is given by

$$
\begin{aligned}
& \dot{n}_{\mathrm{r}}=1-\mu_{1}-2 n_{\mathrm{r}}^{2} \\
& \dot{\mu}_{1}=-2 \mu_{1} n_{\mathrm{r}}
\end{aligned}
$$

which coincides with $(2.3)$ once $n_{\mathrm{r}}$ is identified with $B_{1}$. We see that (2.16) has the singular equilibria

$$
p_{\mathrm{s}}^{ \pm}=\left(n_{\mathrm{r}}, n_{\mathrm{i}}, \mu_{1}\right)=\left( \pm \frac{1}{\sqrt{2}}, 0,0\right)
$$

and the wave equilibria

$$
p_{\mathrm{w}}(k)=\left(n_{\mathrm{r}}, n_{\mathrm{i}}, \mu_{1}\right)=\left(0, k, 1+k^{2}\right) .
$$

Inside the Eckhaus boundary $|k|<1 / \sqrt{2}$, the wave equilibria have one unstable and one stable eigenvalue in addition to the neutral eigenvalue that arises from the parameter $k$. Furthermore, if we identify $n_{\mathrm{r}}$ with $B_{1}$ and set $n_{\mathrm{i}}=0$, then (2.7) shows that the unstable manifold of the equilibrium $p_{\mathrm{w}}(0)$ connects to the sink $p_{\mathrm{s}}^{+}$in such a fashion that $n_{\mathrm{r}}$ increases while $\mu_{1}$ decreases along the heteroclinic connection $n_{\mathrm{i}}$.

In the $\left(a_{1}, b_{1}\right)$-coordinates, the above flow is augmented by an additional neutral phase variable that arises due to the equivariance of the normal form with respect to the action (2.13): each $\left(a_{1}, b_{1}\right)=\left(\mathrm{e}^{\mathrm{i} \phi} a_{1}^{0}, \mathrm{e}^{\mathrm{i} \phi} b_{1}^{0}\right)$ with $\phi \in \mathbb{R}$ corresponds to the same $n=a_{1}^{0} \bar{b}_{1}^{0}$.

As a result, the wave equilibria are relative equilibria with respect to the phase rotation (2.13),

$$
p_{\mathrm{w}}=\left(a_{1}, b_{1}, \mu_{1}\right)=\left(\mathrm{e}^{-\mathrm{i} k y},-\mathrm{i} k \mathrm{e}^{-\mathrm{i} k y}, 1+k^{2}\right) .
$$

They persist for $|A|>0$ as a family of reversible periodic orbits whose unstable manifolds depend smoothly on the parameter $|A|$ which measures the distance to the invariant sphere: the most convenient way to prove this is to use the rescaling chart in which the existence of Turing patterns is well known $[17, \S 3.1]$.

The singular equilibria form two circles $^{1}$ given by

$$
p_{\mathrm{s}}^{ \pm}=\left(a_{1}, b_{1}, \mu_{1}\right)=\left(\mathrm{e}^{\mathrm{i} \phi}, \frac{ \pm 1}{\sqrt{2}} \mathrm{e}^{\mathrm{i} \phi}, 0\right), \quad \phi \in \mathbb{R} .
$$

If we omit the artifical $\psi$-direction, the eigenvalues of the singular equilibrium $p_{\mathrm{s}}^{+}$are

$$
\lambda_{\mathrm{c}}=0, \quad \lambda_{\mathrm{s}}=-2 / \sqrt{2}, \quad \lambda_{\mathrm{ss}} \in\{-3 / \sqrt{2},-4 / \sqrt{2}\}, \quad \lambda_{\mathrm{u}}=1 / \sqrt{2},
$$

where the unstable eigenvector points into the direction normal to the sphere ${ }^{2}$.

For later reference, we calculate the expansion of the unstable manifold of $p_{\mathrm{s}}^{+}$: Since its unstable eigenvector points into the $|A|$-direction, the unstable manifold is given by

$$
W_{\mathrm{loc}}^{\mathrm{u}}\left(p_{\mathrm{s}}^{+}\right)=\left\{\left(a_{1}, b_{1},|A|\right)=\left(\mathrm{e}^{\mathrm{i} \phi}+\mathrm{O}\left(r^{2}\right), \frac{1}{\sqrt{2}} \mathrm{e}^{\mathrm{i} \phi}+\mathrm{O}\left(r^{2}\right), r\right) ;|r| \ll 1\right\} .
$$

\footnotetext{
${ }^{1}$ Cylinders, in fact, if we take the artificial $\psi$-direction into account.

${ }^{2}$ The eigenvalues of the singular equilibrium $p_{\mathrm{s}}^{-}$have the same modulus but the opposite sign.
} 
Transforming this expansion back to the original coordinates $(A, B)$, we obtain

$$
W_{\mathrm{loc}}^{\mathrm{u}}(0)=\left\{(A, B)=\left(a_{1}|A|, b_{1}|A|^{2}\right)=\left(r \mathrm{e}^{\mathrm{i} \phi}, \frac{r^{2} \mathrm{e}^{\mathrm{i} \phi}}{\sqrt{2}}\right)+\mathrm{O}\left(r^{3}\right) ;|r| \ll 1\right\} .
$$

The next step is to track the unstable manifolds $W^{\mathrm{u}}\left(p_{\mathrm{w}}(|A|)\right)$ of the wave equilibria for small positive $|A|$ as they pass near the singular equilibria and to compare the distance of $W^{\mathrm{u}}\left(p_{\mathrm{w}}(|A|)\right)$ after passage to the unstable manifolds of the singular equilibria, measured at a finite distance

$|A|=\delta$ from the singular equilibria. We analyse this transition in Appendix $\mathrm{B}$ as it is more involved. The result proved in Appendix B states that the distance of the unstable manifold $W^{\mathrm{u}}\left(p_{\mathrm{w}}(|A|)\right)$ of the wave equilibria from the unstable manifold of the singular equilibria, measured at a finite distance $|A|=\delta$ from the singular equilibria, is of order $\mathrm{O}(\mu)$.

This proves that the unstable manifold of the small-amplitude periodic patterns inside the Eckhaus stability boundary is close to the singular unstable manifold for sufficiently small $\mu>0$. The rest of the proof of Theorem 3 is completely analogous to the one given in Section 2.1 for pitchfork bifurcations: First, extend the above statement to $\mu<0$. Afterwards, the unstable manifolds in the full phase space are obtained by complementing the unstable manifolds inside the center manifold with the strong unstable fibers of the center manifold. Transversality of the centerunstable manifold and the fixed-point space of the reverser follows as in Section 2.1 since solutions in the singular unstable manifold of the origin decay again algebraically for $\mu=0$ with their asymptotics given by $|A|=\sqrt{2} / x+\mathrm{O}\left(1 / x^{2}\right)$.

\subsection{Oscillatory instabilities: Existence}

We seek time-periodic pulses of

$$
u_{t}=D u_{x x}+f(u ; \mu)
$$

with temporal frequency $\omega$. Using the new time variable $\tau$ with $t=\omega \tau$, these can be found as solutions to the modulated-wave equation

$$
\begin{aligned}
& u_{x}=v \\
& v_{x}=D^{-1}\left[\omega \partial_{\tau} u-f(u ; \mu)\right]
\end{aligned}
$$

where $(u, v)(x) \in Y=H^{1 / 2}\left(S^{1}, \mathbb{R}^{2 n}\right) \times L^{2}\left(S^{1}, \mathbb{R}^{2 n}\right)$ are $2 \pi$-periodic functions of $\tau$ for each fixed $x \in \mathbb{R}$. We view (2.19) as a dynamical system in the spatial variable $x$ where the temporal frequency $\omega \approx \omega_{*}$ is a parameter. Our strategy of finding oscillatory pulses follows closely the one employed for pitchfork and Turing instabilities.

As a dynamical system on $Y,(2.19)$ is equivariant with respect to the $S^{1}$-action generated by the temporal time-shift $\tau \mapsto \tau+\check{\tau}$ on $Y$ and reversible with respect to the linear reversers

$$
\begin{array}{lll}
\mathcal{R}_{0}: & Y \longrightarrow Y & {\left[\mathcal{R}_{0}(u, v)\right](\tau)=(u,-v)(\tau)} \\
\mathcal{R}_{\pi}: & Y \longrightarrow Y & {\left[\mathcal{R}_{\pi}(u, v)\right](\tau)=(u,-v)(\tau+\pi) .}
\end{array}
$$


Note that $\mathcal{R}_{0}$ detects symmetric modulated pulses, while $\mathcal{R}_{\pi}$ seeks out the asymmetric modulated waves shown in Figure 3. The action of the reversers on

$$
Y_{\ell}=\left\{\mathrm{e}^{\mathrm{i} \ell \tau}\left(u_{0}, v_{0}\right) ;\left(u_{0}, v_{0}\right) \in \mathbb{R}^{2 n}\right\} \subset Y, \quad \ell \in \mathbb{Z}
$$

is given by

$$
\begin{aligned}
\mathcal{R}_{0}: & Y_{\ell} \longrightarrow Y_{\ell} & \left(u_{0}, v_{0}\right) & \longmapsto\left(u_{0},-v_{0}\right) \\
\mathcal{R}_{\pi}: & Y_{\ell} \longrightarrow Y_{\ell} & \left(u_{0}, v_{0}\right) & \longmapsto(-1)^{\ell}\left(u_{0},-v_{0}\right) .
\end{aligned}
$$

In particular, solutions with initial data in $\operatorname{Fix}\left(\mathcal{R}_{0}\right)$ are even in $x$, while solutions with initial data in $\operatorname{Fix}\left(\mathcal{R}_{\pi}\right) \cap Y_{\ell}$ are even in $x$ for even $\ell$ and odd in $x$ for odd $\ell$.

The fixed-point subspace of the $S^{1}$-action is $Y_{0}$, which consists precisely of all functions $(u, v) \in Y$ that do not depend on $\tau$, and the dynamics of (2.19) on $Y_{0}$ is given by the dynamics of the usual standing-wave ODE (1.3). In particular, Hypothesis 11 states that, inside this fixed-point subspace, there exists a homoclinic orbit $\left(q, q^{\prime}\right)(x)$ to the hyperbolic equilibrium $(u, v)=0$, and $\left(q, q^{\prime}\right)(x)$ decays exponentially as $\xi \rightarrow \pm \infty$.

With the help of [16], we infer from Hypothesis 7 that the equilibrium $(u, v)=0$ has a fourdimensional, locally invariant center manifold $W^{\mathrm{c}}(0)$ in the space $Y$ and an infinite-dimensional center-unstable manifold $W^{\mathrm{cu}}(0)$ which is fibered smoothly over $W^{\mathrm{c}}(0)$. Furthermore, we know from [16] that the invariant manifolds and their fibers depend smoothly on $\mu$ and that all solutions to (2.19) that remain close to $(u, v)=0$ as $x \rightarrow-\infty$ lie necessarily in the center-unstable manifold $W^{\mathrm{cu}}(0)$.

We claim that the center-unstable manifold $W^{\mathrm{cu}}(0)$ at the bifurcation point $\mu=0$ intersects both $\operatorname{Fix}\left(\mathcal{R}_{0}\right)$ and $\operatorname{Fix}\left(\mathcal{R}_{\pi}\right)$ transversely along two-dimensional manifolds that are invariant under the $S^{1}$ action introduced above. To prove this claim, we first note that the intersection contains the point $\left(q(0), q^{\prime}(0)\right)$ which lies in $\operatorname{Fix}\left(S^{1}\right)$. Furthermore, Fredholm theory [21] shows that the intersection is at least two-dimensional. We now argue by contradiction and assume that the intersection of the tangent space of $W^{\text {cu }}(0)$ at $\left(q, q^{\prime}\right)(0)$ with either $\operatorname{Fix}\left(\mathcal{R}_{0}\right)$ or $\operatorname{Fix}\left(\mathcal{R}_{\pi}\right)$ in $Y$ is of dimension equal to at least three. In particular, this intersection provides a three-dimensional space of reversible solutions of the linearization

$$
\begin{aligned}
& u_{x}=v \\
& v_{x}=D^{-1}\left[\omega_{*} \partial_{\tau} u-f_{u}(q(x) ; 0) u\right]
\end{aligned}
$$

of (2.19) about $\left(q, q^{\prime}\right)(\xi)$ with $\omega=\omega_{*}$. On the other hand, using that the homoclinic orbit converges to zero exponentially and exploiting Hypothesis 7 in the same fashion as in $[16, \S 3.3]$, we see that the space of initial data in $T_{\left(q, q^{\prime}\right)(0)} W^{\mathrm{cu}}(0)$ that lead to solutions that are bounded for $x \rightarrow-\infty$ is of codimension two: it consists of the strong unstable subspace and the two-dimensional space of solutions that converge to constants as $x \rightarrow-\infty$. Since we started with a three-dimensional subspace of initial data, we therefore obtain at least one nonzero reversible bounded solution of (2.21). Since the spaces $Y_{\ell}$ are invariant under (2.21), [16, Lemma 3.4] and the statement following 
(2.20) imply that any such solution corresponds to an element in the null space of $\mathcal{L}_{*}-\mathrm{i} \omega_{*} \ell$ on $C_{\text {even }}^{0}\left(\mathbb{R}, \mathbb{C}^{n}\right)$ for some $\ell \in \mathbb{Z}$ or on $C_{\text {odd }}^{0}\left(\mathbb{R}, \mathbb{C}^{n}\right)$ for some odd $\ell \in \mathbb{Z}$. This, however, contradicts Hypothesis 11, and therefore proves our original claim.

The transversality statement that we just proved allows us to pull back the $\mu$-dependent intersections $W^{\text {cu }}(0 ; \mu) \cap \operatorname{Fix}\left(R_{j}\right)$ for $j=0, \pi$ along the strong unstable fibration and project them onto the center manifold $W^{\mathrm{c}}(0 ; \mu)$. This procedure results in two surfaces $\Gamma_{0}(\mu)$ and $\Gamma_{\pi}(\mu)$ that are contained in $W^{\mathrm{c}}(0 ; \mu)$ and depend smoothly on $\mu$. As a consequence, any solution of (2.19) within the center manifold $W^{\mathrm{c}}(0 ; \mu)$ that intersects $\Gamma_{0}(\mu)$ (or $\Gamma_{\pi}(\mu)$ ) can be lifted uniquely to a solution in the center-unstable $W^{\mathrm{c}}(0 ; \mu)$ whose trajectory intersects $\operatorname{Fix}\left(R_{0}\right)$ (or $\left.\operatorname{Fix}\left(R_{\pi}\right)\right)$. Thus, we restrict our attention to the center manifold and construct solutions that intersect one of the surfaces $\Gamma_{j}(\mu)$ with $j=0, \pi$.

The vector field on the four-dimensional center manifold has been derived in [25, §2.4.3]: If $u_{\mathrm{h}}(x)$ denotes the complex Hopf eigenfunction of $\mathcal{L}_{*}$ belonging to the eigenvalue $\mathrm{i} \omega_{*}$, then we can parametrize the generalized Hopf eigenspace of $\mathcal{L}_{*}$ via $(u, v)=\left(a \mathrm{e}^{\mathrm{i} \tau} u_{\mathrm{h}}, b \mathrm{e}^{\mathrm{i} \tau} u_{\mathrm{h}}\right)$ where $(a, b) \in \mathbb{C}^{2}$ are complex coordinates. Using these coordinates to parametrize the center manifold, we recover, to leading order, the unscaled version of the complex Ginzburg-Landau equation (1.8). In fact, as shown in $[25, \S 2.4 .3]$, the vector field on $W^{\mathrm{c}}(0 ; \mu)$ is given by

$$
\begin{aligned}
& a_{x}=b \\
& b_{x}=\frac{1}{1+\mathrm{i} \alpha}\left[-(\mu+\mathrm{i} \check{\omega}) a+(1+\mathrm{i} \beta)|a|^{2} a+\mathrm{O}\left(\left|a b^{2}\right|+|a|^{5}+|b|^{5}\right)\right],
\end{aligned}
$$

where the coefficients $\alpha, \beta$ are as in Section 1.4 and where we wrote $\omega=\omega_{*}+\check{\omega}$ for the temporal frequency $\omega$ appearing in (2.19).

Since we wish to find solutions to $(2.22)$ that intersect the surfaces $\Gamma_{j}(\mu)$, we need to find representations of $\Gamma_{j}(\mu)$ in terms of $(a, b)$. First, since the $\tau$-independent primary pulse $q$ persists in $Y_{0}$, the surfaces $\Gamma_{j}(\mu)$ intersect the origin $(a, b)=0$ for all $\mu$ small. Each surface is also invariant under the action of the temporal $\tau$-shift symmetry $(a, b) \mapsto\left(\mathrm{e}^{\mathrm{i} \phi} a, \mathrm{e}^{\mathrm{i} \phi} b\right)$. Lastly, exploiting again Hypothesis 11, each surface intersects the line $b=0$ transversely, since the null space of the operator $\mathcal{L}_{*}-\mathrm{i} \omega_{*}$ would otherwise be nontrivial. For $j=0, \pi$, we can therefore write the surfaces $\Gamma_{j}$ as graphs

$$
a=\rho_{j}(\mu) b+\mathrm{O}\left(|b|^{3}\right)
$$

for certain smooth functions $\rho_{j}(\mu)$.

We have now collected the information necessary for proceeding with the blow-up of (2.22). Instead of a full-fledged blow-up, it suffices to consider the rescaling chart for $\mu>0$ whose coordinates scale according to

$$
a=\sqrt{\mu} A, \quad b=\mu B, \quad \check{\omega}=\mu \Omega, \quad x=\frac{X}{\sqrt{\mu}} .
$$

In these coordinates ${ }^{3},(2.22)$ becomes

$$
A_{X}=B
$$

\footnotetext{
${ }^{3}$ We break here with the convention used in the previous sections that capital letters denote the coordinates before blow-up to keep in line with the usual conventions for the variables in the CGL.
} 


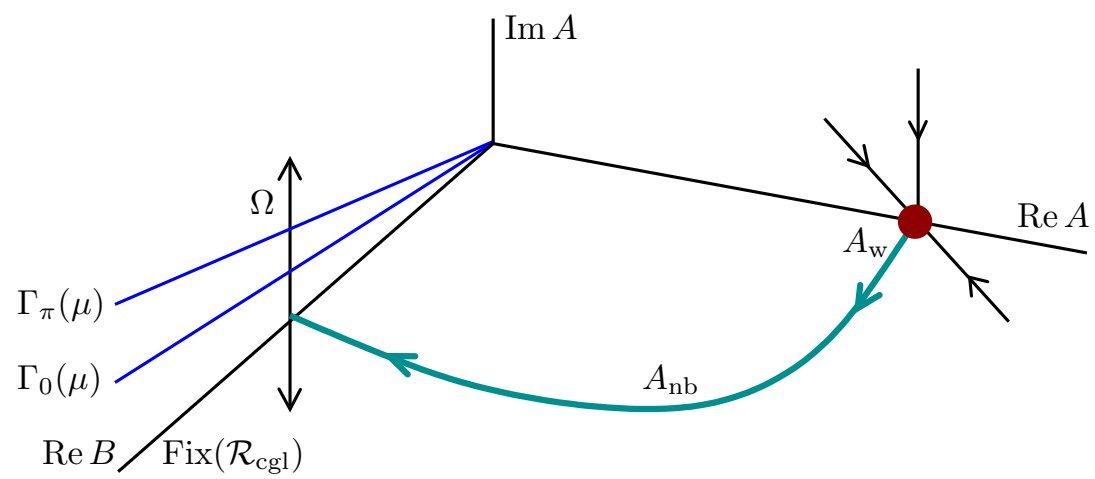

Figure 7: The geometry in phase space of the Nozaki-Bekki hole and the reversibility surfaces $\Gamma_{j}(\mu)$ for the rescaled complex Ginzburg-Landau equation is shown.

$$
B_{X}=\frac{1}{1+\mathrm{i} \alpha}\left[-(1+\mathrm{i} \Omega) A+(1+\mathrm{i} \beta)|A|^{2} A+\mathrm{O}(\mu)\right]
$$

while the reversibility surfaces $\Gamma_{j}(\mu)$ are now given by

$$
\Gamma_{j}(\mu)=\left\{(A, B) ; A=\sqrt{\mu} \rho_{j}(0) B+\mathrm{O}(|\mu B|)\right\}, \quad j=0, \pi
$$

For $\mu=0$, we see that (2.25) becomes (1.9), while the reversibility surfaces $\Gamma_{j}(0)$ are then both equal to $\operatorname{Fix}\left(\mathcal{R}_{\text {cgl }}\right)$, where $\mathcal{R}_{\text {cgl }}(A, B)=(-A, B)$ is the reverser for (1.9). We can now exploit Hypothesis 9 where we assumed that the Nozaki-Bekki hole $A_{\mathrm{nb}}(X)$ connects the wave train $A_{\mathrm{w}}\left(X ; \kappa_{*}\right)$ with wavenumber $\kappa_{*}$ at $X=-\infty$ to $\operatorname{Fix}\left(\mathcal{R}_{\mathrm{cgl}}\right)$ at $X=0$ provided we choose $\Omega=\Omega_{*}=\Omega_{\mathrm{nl}}\left(\kappa_{*}\right)$. We also assumed that the intersection $A_{\mathrm{nb}}(X)$ of $W^{\mathrm{cu}}\left(A_{\mathrm{w}}\left(\cdot ; \kappa_{*}\right)\right)$ and $\operatorname{Fix}\left(\mathcal{R}_{\mathrm{cgl}}\right)$ is transverse with respect to varying the real parameter $\Omega$ near $\Omega_{*}$ (see Figure 7 ).

Thus, since $\Gamma_{j}(0)=\operatorname{Fix}\left(\mathcal{R}_{\text {cgl }}\right)$ at $\mu=0$, we can find, for each small $\mu>0$, a number $\Omega$ close to $\Omega_{*}$ such that the intersection between $W^{\mathrm{cu}}\left(A_{\mathrm{w}}\right)$ and $\Gamma_{j}(\mu)$ is non-empty: we denote the resulting solution by $A_{\mathrm{nb}}(X ; \mu)$. Since the periodic orbit $A_{\mathrm{w}}(X)$ of $(1.9)$ with $\mu=0$ has a simple Floquet exponent at zero by Hypothesis 9 , it persists in the center manifold for all small $\mu>0$. Thus, the connection $A_{\mathrm{nb}}(X ; \mu)$ that we constructed above converges indeed to a spatially-periodic orbit with wavenumber close to $\kappa_{*}$. The first component of the corresponding reversible heteroclinic orbit $Q_{j}(x, \tau ; \mu)$ to $(2.19)$ is the oscillating pulse $q_{j}(x, \tau ; \mu)$ of Theorem 5 . The expansions for wavenumber and temporal frequency can be obtained by inverting the coordinate transformation (2.24) and recalling, in particular, that the spatial variable has also been rescaled in (2.24).

For later use, we remark that

$$
\left(a_{\mathrm{nb}}, b_{\mathrm{nb}}\right)(0 ; \mu)=\mathrm{O}(\mu), \quad\left(\frac{\mathrm{d} a_{\mathrm{nb}}}{\mathrm{d} x}, \frac{\mathrm{d} b_{\mathrm{nb}}}{\mathrm{d} x}\right)(0 ; \mu)=\left(\mathrm{O}(\mu), \mathrm{O}\left(\mu^{2}\right)\right),
$$

where $\left(a_{\mathrm{nb}}, b_{\mathrm{nb}}\right)$ corresponds to the solution $A_{\mathrm{nb}}(X ; \mu)$ under the scaling $(2.24)$. Furthermore, we have

$$
\sup _{x \in[-L, 0]}\left\|q_{0}(x, \tau ; \mu)-q(x)\right\|_{Y}=\mathrm{O}(\mu)
$$

for each fixed $L \gg 1$. 


\section{Stability for pitchfork and Turing bifurcations}

Our strategy to prove asymptotic or spectral stability of the bifurcating standing pulses will be basically identical for pitchfork and for Turing instabilities. First, we locate the essential spectra of the bifurcating pulses. Afterwards, we need to exclude the possibility that isolated eigenvalues emerge from the essential spectrum and move into the right half-plane during the bifurcation. To exclude these edge bifurcations, we decompose the phase space into three different subspaces: the strong stable and unstable subspaces consist of all initial data that lead to solutions of the linearized equation which decay to zero exponentially with a uniform rate as $x \rightarrow \infty$ and $x \rightarrow-\infty$, respectively. Solutions to initial data that lie in the remaining complementary center subspace decay or grow algebraically, or at most exponentially with a small rate, as $|x| \rightarrow \infty$. These three subspaces depend smoothly on the prospective eigenvalue parameter $\lambda$ and on the bifurcation parameter $\mu$. The issue is then to construct a splitting of the center space into two subspaces of equal dimension that continue the unstable and stable subspaces through the bifurcation point and that depend again smoothly on $\lambda$ and $\mu$. Once this splitting is obtained, spectral stability is a straightforward consequence of the fact that the simple eigenvalue at zero remains at the origin due to translational invariance.

The smooth continuation of the splitting in the center bundle relies on the same blow-up technique that we employed when proving existence: we construct the two subspaces by establishing the existence of certain singular heteroclinic orbits between the rescaling and the singular chart and tracking these orbits as they pass near the singular equilibrium. This procedure can be interpreted as a factorization of the Evans function for the bifurcating pulse into a product of the nonzero singular Evans function of the singular heteroclinic orbit and the Evans function of the standing pulse at the bifurcation point. We emphasize that these constructions are not standard as the pulse $q(x)$ decays only algebraically at the bifurcation point, and techniques such as the Gap Lemma fail in this situation. We mention that we used similar ideas in [20] to which we refer for additional details.

\subsection{Essential spectra}

The essential spectrum of the bifurcating pulses is given by the essential spectra of the asymptotic states. For pitchfork bifurcations, the spectrum of the asymptotic bifurcating equilibria is given, to leading order in $\mu$, by

$$
\lambda_{\mathrm{pf}}(k ; \mu)=\lambda(0 ; \mu)+\frac{1}{2} \lambda_{k k}(0 ; 0) k^{2}+\mathrm{O}\left(|\mu| k^{2}+k^{4}\right) .
$$

Exploiting that the kinetic ODE (1.4) has a stable eigenvalue in the center manifold, we see that $\lambda(0 ; \mu)<0$ for $\mu \neq 0$. Since, by assumption, $\lambda_{k k}(0 ; 0)<0$, we conclude that the essential spectrum of the standing pulse is contained in the open left half-plane for $\mu \neq 0$.

The analysis for Turing instabilities has been carried out in [17, §3.3]: For $\mu>0$, the essential spectrum near $\lambda=0$ consists of two curves: one of these curves lies in the open left half-plane, 
while the second one can be parametrized by

$$
\lambda_{\text {turing }}(\gamma ; \mu)=\frac{1}{2} \lambda_{k k}(0 ; 0) \mu^{2} \gamma^{2}+\mathrm{O}\left(|\mu \gamma|^{3}\right)
$$

where $\lambda_{k k}(0 ; 0)<0$ inside the Eckhaus stable band.

\subsection{The Evans function}

To prove Theorem 2, it therefore suffices to exclude spectrum in $\operatorname{Re} \lambda \geq 0$ with the exception of an algebraically simple eigenvalue at the origin $\lambda=0$. We rewrite the eigenvalue problem

$$
D u_{x x}+f_{u}(q(x ; \mu) ; \mu) u=\lambda u
$$

for the linearization at the pulse $q(x ; \mu)$ as

$$
\begin{aligned}
& u_{x}=v \\
& v_{x}=D^{-1}\left[\lambda-f_{u}(q(x ; \mu) ; \mu)\right] u .
\end{aligned}
$$

Hypothesis 4 and regular perturbation theory imply that any potential eigenvalue $\lambda$ with $\operatorname{Re} \lambda \geq 0$ lies inside a small neighborhood $|\lambda| \leq \delta$ of the origin. Furthermore, a complex number $\lambda$ belongs to the point spectrum if, and only if, there exists a nontrivial bounded solution $(u, v)$ to $(3.1)$.

For each $\lambda$ to the right of the essential spectrum (i.e. in the connected component of the resolvent set that contains the positive real axis), there exist unique analytic families of $n$-dimensional subspaces $E_{-}^{\mathrm{u}}(\lambda ; \mu)$ and $E_{+}^{\mathrm{s}}(\lambda ; \mu)$ that consist precisely of those initial data that lead to solutions of $(3.1)$ which are bounded on $\mathbb{R}_{-}$and on $\mathbb{R}_{+}$, respectively. The point spectrum is therefore given by the set of all $\lambda$ to the right of the essential spectrum for which $E_{-}^{\mathrm{u}}(\lambda ; \mu)$ and $E_{+}^{\mathrm{s}}(\lambda ; \mu)$ intersect nontrivially. Choosing bases $\left(e_{j}^{\mathrm{u}}\right)_{1 \leq j \leq n}$ and $\left(e_{j}^{\mathrm{S}}\right)_{1 \leq j \leq n}$ of these two spaces that depend analytically on $\lambda$ and are continuous in $\mu$, we see that the point spectrum clearly coincides with the roots $\lambda$ of the analytic function

$$
\mathcal{E}(\lambda ; \mu)=\operatorname{det}\left[e_{1}^{\mathrm{u}}, \ldots, e_{n}^{\mathrm{u}}, e_{1}^{\mathrm{s}}, \ldots, e_{n}^{\mathrm{s}}\right]
$$

for each fixed $\mu$. Spectral stability of the bifurcating pulses will be a consequence of the following two propositions which we will in $§ 3.3-3.4$.

Proposition 3.1 For both pitchfork and Turing instabilities, the bundles $E_{-}^{\mathrm{u}}(\lambda ; \mu)$ and $E_{+}^{\mathrm{s}}(\lambda ; \mu)$ for the bifurcating pulses can be extended continuously in $\mu$ as $C^{2}$-functions of $\gamma=\sqrt{\lambda}$ into $\operatorname{Re} \gamma \geq 0$. To the right of the essential spectrum, the bundles $E_{-}^{\mathrm{u}}(\lambda ; \mu)$ and $E_{+}^{\mathrm{s}}(\lambda ; \mu)$ consists precisely of all solutions to (3.1) that are bounded on $\mathbb{R}^{-}$and $\mathbb{R}^{+}$, respectively. In particular, the Evans function $\check{\mathcal{E}}(\gamma ; \mu):=\mathcal{E}\left(\gamma^{2} ; \mu\right)$ is $C^{2}$ in $\gamma$ and $C^{0}$ in $\mu$.

Proposition 3.2 For both pitchfork and Turing instabilities, the Evans function $\mathcal{E}(\lambda ; \mu)$ is $C^{1}$ in $\lambda$ for $\operatorname{Re} \lambda \geq 0$, and we have $\mathcal{E}(0 ; 0)=0$ and $\mathcal{E}_{\lambda}(0 ; 0) \neq 0$. 
Proof of Theorems 2 and 4. Using the bundles constructed in Proposition 3.1, we extend the Evans function $\check{\mathcal{E}}(\gamma ; \mu)$ as a $C^{0}$-function in $\mu$ and a $C^{2}$-function in $\gamma$ to a neighborhood of $\gamma=0$ in $\operatorname{Re} \gamma \geq 0$. We also see that $\mathcal{E}(\lambda ; \mu)$ is $C^{1}$ in $\lambda$ for $\operatorname{Re} \lambda \geq 0$. Since $\mathcal{E}(\lambda, 0)$ has a simple root at the origin, the implicit function theorem guarantees that there exists a unique root of $\mathcal{E}(\lambda ; \mu)$ in a neighborhood of the origin for all $\mu$ close to zero. Since the derivative $\partial_{x} q(x ; \mu)$ of the bifurcating pulses supply a bounded solution of (3.1) with $\lambda=0$, this root stays at $\lambda=0$ for all $\mu$, and we conclude that there does not exist any unstable spectrum in a neighborhood of the origin. This proves Theorems 2 and 4 .

We will suppress the dependence on $\mu$. Using exponential dichotomies, we conclude as in $[17,20]$ that there are analytic families of subspaces $E_{-}^{\mathrm{cu}}(\lambda), E_{+}^{\mathrm{cs}}(\lambda) \subset \mathbb{C}^{2 n}$, which are given as ranges of analytic projections $P_{-}^{\mathrm{cu}}(\lambda)$ and $P_{+}^{\mathrm{cs}}(\lambda)$, such that initial data at $x=0$ to solutions of (3.1) that decay as $x \rightarrow \infty$ lie in $E_{+}^{\mathrm{cs}}(\lambda)$, while initial data to solutions that decay as $x \rightarrow-\infty$ lie in $E_{-}^{\mathrm{cu}}(\lambda)$. The dimensions of these subspaces are $n+1$ for pitchfork bifurcations and $n+2$ for Turing instabilities. In general, solutions in $E_{+}^{\mathrm{cs}}(\lambda)$ may grow exponentially as $x \rightarrow \infty$, but they do so only with a small exponential rate as long as $\lambda$ is small. Similarly, solutions in $E_{-}^{\text {cu }}$ may grow exponentially with a small rate as $x \rightarrow-\infty$. We can write these subspaces as direct sums of analytic subspaces

$$
E_{-}^{\mathrm{cu}}(\lambda)=E_{-}^{\mathrm{c}}(\lambda) \oplus E_{-}^{\mathrm{uu}}(\lambda), \quad E_{+}^{\mathrm{cs}}(\lambda)=E_{+}^{\mathrm{c}}(\lambda) \oplus E_{+}^{\mathrm{ss}}(\lambda) .
$$

The subspaces $E_{+}^{\mathrm{ss}}(\lambda)$ and $E_{-}^{\mathrm{uu}}(\lambda)$ have dimension $n-1$ for pitchfork bifurcations and $n-2$ for Turing instabilities, and solutions with initial data in $E_{+}^{\mathrm{ss}}(\lambda)$ and $E_{-}^{\mathrm{uu}}(\lambda)$ decay exponentially as $x \rightarrow \infty$ and $x \rightarrow-\infty$, respectively, with a rate that is independent of $\lambda$.

Thus, it suffices to construct a further splitting of the center bundles $E_{ \pm}^{c}(\lambda)$ that extends the spaces $E_{-}^{\mathrm{c}}(\lambda) \cap E_{-}^{\mathrm{u}}(\lambda)$ and $E_{+}^{\mathrm{c}}(\lambda) \cap E_{+}^{\mathrm{s}}(\lambda)$ from $\operatorname{Re} \lambda>0$ into $\operatorname{Re} \gamma \geq 0$. This construction will be carried out first for pitchfork bifurcations. Afterwards, we explain the modifications that are necessary to treat Turing instabilities.

\subsection{Pitchfork bifurcations: Stability}

To find the desired splitting of the center bundle, we consider the nonlinear and the linearized system simultaneously:

$$
\begin{aligned}
& u_{x}=v \\
& v_{x}=-D^{-1} f(u ; \mu) \\
& \check{u}_{x}=\check{v} \\
& \check{v}_{x}=D^{-1}\left[\lambda-f_{u}(u ; \mu)\right] \check{u} .
\end{aligned}
$$

We are interested in bounded solutions of the last two equations in $(3.2)$ when $u(x ; \mu)$ is the homoclinic orbit found in Theorem 1. Note that (3.2) has a natural skew-product structure since 
the equations for $(u, v)$ do not depend on $(\check{u}, \check{v})$. For $(\mu, \lambda)$ close to zero, the origin has a fourdimensional center manifold. Within this invariant manifold, we recover the skew-product structure. Furthermore, after transforming variables appropriately, the vector field on the center manifold is given by

$$
\begin{aligned}
A_{x} & =B \\
B_{x} & =-\mu A+A^{3}+A \mathrm{O}\left(|A|^{4}+|B|^{4}+|\mu||A|^{2}+\mu^{2}|A|\right) \\
a_{x} & =b \\
b_{x} & =-\mu a+3 A^{2} a+\lambda a+\mathrm{O}\left(|\lambda|^{2}+|A|^{3}+|B|^{3}+|\mu||A|+\mu^{2}\right)(|a|+|b|) .
\end{aligned}
$$

If we set

$$
\mu=\mu_{0} \varepsilon, \quad \lambda=\gamma_{0}^{2} \varepsilon
$$

with $\operatorname{Re} \gamma_{0} \geq 0$, and use the coordinate $z=b / a$ in the projective space, we obtain

$$
\begin{aligned}
& A_{x}=B \\
& B_{x}=-\mu_{0} \varepsilon A+A^{3}+A \mathrm{O}\left(|A|^{4}+|B|^{4}+|\varepsilon||A|^{2}+\varepsilon^{2}|A|\right) \\
& z_{x}=3 A^{2}+\left(\gamma_{0}^{2}-\mu_{0}\right) \varepsilon-z^{2}+\mathrm{O}\left(\varepsilon^{2}+|A|^{3}+|B|^{3}+\varepsilon|A|\right) .
\end{aligned}
$$

\section{Singular chart: Small eigenvalues}

We begin by investigating small eigenvalues $|\lambda| \leq \mu$ in the supercritical regime $\mu>0$. Thus, we set $\mu_{0}=1$ and $\operatorname{Re} \gamma_{0} \geq 0$ with $\left|\gamma_{0}\right|^{2} \leq 1$, and employ again the blow-up procedure from Section 2.1. Upon defining

$$
A_{1}=A, \quad B_{1}=\frac{B}{A^{2}}, \quad \varepsilon_{1}=\frac{\varepsilon}{A^{2}}, \quad z_{1}=\frac{z}{A},
$$

and after factoring the Euler multiplier $A_{1}$, equation (3.4) becomes

$$
\begin{aligned}
A_{1}^{\prime} & =A_{1} B_{1} \\
B_{1}^{\prime} & =1-\varepsilon_{1}-2 B_{1}^{2}+\mathrm{O}\left(A_{1}\right) \\
\varepsilon_{1}^{\prime} & =-2 \varepsilon_{1} B_{1} \\
z_{1}^{\prime} & =3+\left(\gamma_{0}^{2}-1\right) \varepsilon_{1}-B_{1} z_{1}-z_{1}^{2}+\mathrm{O}\left(A_{1}\right) .
\end{aligned}
$$

Equation (3.5) has the two singular equilibria

$$
p_{\mathrm{s}}^{+}=\left(0, \frac{1}{\sqrt{2}}, 0, \sqrt{2}\right), \quad p_{\mathrm{s}}^{-}=\left(0, \frac{1}{\sqrt{2}}, 0, \frac{-3}{\sqrt{2}}\right)
$$

in the coordinates $\left(A_{1}, B_{1}, \varepsilon_{1}, z_{1}\right)$. The singular equilibrium $p_{\mathrm{s}}^{+}$is exponentially attracting inside the singular sphere $A_{1}=0$ with stable eigenvalues $-2 / \sqrt{2},-4 / \sqrt{2}$ and $-5 / \sqrt{2}$, while it is unstable in the direction $A_{1}$ normal to the sphere with unstable eigenvalue $1 / \sqrt{2}$. The eigenvector $e_{\mathrm{s}}$ and the associated adjoint eigenvector $e_{\mathrm{s}}^{*}$ that correspond to the leading stable eigenvalue $-2 / \sqrt{2}$ are given by

$$
e_{\mathrm{s}}=\left(0, \frac{-1}{\sqrt{2}}, 1, \frac{\sqrt{2} \gamma_{0}^{2}}{3}\right), \quad e_{\mathrm{s}}^{*}=(0,0,1,0)
$$


We now set $A_{1}=0$. In addition to the singular equilibria $p_{\mathrm{s}}^{ \pm}$, we find the wave equilibria

$$
p_{\mathrm{w}}^{ \pm}=\left(0,0,1, \pm \sqrt{2+\gamma_{0}^{2}}\right) .
$$

They have a one-dimensional unstable manifold whose $\left(B_{1}, \varepsilon_{1}\right)$-component contains the explicit heteroclinic solution $(2.7)$

$$
\left(B_{1}, \varepsilon_{1}\right)(x)=\left(\frac{\sqrt{2}}{4}\left(1+\tanh \left(\frac{x}{\sqrt{2}}\right)\right), \frac{1}{2}\left(1-\tanh \left(\frac{x}{\sqrt{2}}\right)\right)\right)
$$

or, equivalently,

$$
B_{1}(x)=\frac{1-\varepsilon_{1}(x)}{\sqrt{2}}, \quad \varepsilon_{1}(x)=\frac{1}{1+\mathrm{e}^{\sqrt{2} x}} .
$$

Note that $0<\varepsilon_{1}(x)<1$ for all $x$. The expression for the $z_{1}$-component of the unstable manifold of $p_{\mathrm{w}}^{ \pm}$is less explicit. It is straightforward to check, however, that $\operatorname{Re} z_{1}^{\prime}>0$ whenever $\operatorname{Re} z_{1}=0$ : indeed, using Re $z_{1}=0,\left|\gamma_{0}^{2}\right| \leq 1$, and $0<\varepsilon_{1}<1$ in the last equation of (3.5), we obtain

$$
\operatorname{Re} z_{1}^{\prime}=3+\left(\operatorname{Re} \gamma_{0}^{2}-1\right) \varepsilon_{1}(x)-\operatorname{Re}\left(z_{1}^{2}\right) \geq 1+\left|z_{1}\right|^{2}>0 .
$$

Thus, the heteroclinic orbit from $p_{\mathrm{w}}^{+}$with $\operatorname{Re} \sqrt{2+\gamma_{0}^{2}}>0$ actually connects to the singular equilibrium $p_{\mathrm{S}}^{+}$with $\operatorname{Re} z_{1}>0$ (see $[20, \S 3.4]$ for details). We remark that the last equation in (3.5) corresponds to the singular Sturm-Liouville eigenvalue problem

$$
a^{\prime \prime}=\left[3+\left(\gamma_{0}^{2}-1\right) \varepsilon_{1}(x)\right] a-B_{1}(x) a^{\prime} .
$$

The conclusion we derived from the estimate (3.8), namely that the unstable manifold of the wave equilibrium $p_{\mathrm{w}}^{+}$connects to the singular equilibrium $p_{\mathrm{s}}^{+}$, is in fact equivalent to the absence of nontrivial eigenvalues $\gamma_{0}$ for the singular Sturm-Liouville problem as a connecting orbit to $p_{\mathrm{s}}^{-}$ would generate such an eigenvalue of the singular Sturm-Liouville problem.

Next, we consider the region $A_{1}>0$ outside the singular sphere $A_{1}=0$. We see that the wave equilibria

$$
p_{\mathrm{w}}^{ \pm}=\left(A_{1}, 0,1+\mathrm{O}\left(A_{1}\right), \pm \sqrt{2+\gamma_{0}^{2}}+\mathrm{O}\left(A_{1}\right)\right)
$$

and their unstable manifolds can be continued uniquely for all small $A_{1}$. For positive $A_{1}$ and $\operatorname{Re} \sqrt{2+\gamma_{0}^{2}}>0$, the equilibrium $p_{\mathrm{w}}^{+}$represents the unstable subspace of the linearization about the asymptotic state of the bifurcating wave.

We fix a small positive constant $\delta>0$. We are then interested in the value $z_{1}^{\text {out }}$ of $z_{1}$ upon tracking the unstable manifold of the wave equilibria that correspond to small $A_{1}$ with $\delta \gg A_{1}>0$ until it hits the cross-section $A_{1}=\delta$. The computation of $z_{1}^{\text {out }}$ requires again an analysis of the passage near the singular equilibrium $p_{\mathrm{s}}^{+}$. Hence, we define two affine cross-sections $\Sigma^{\text {in }}$ and $\Sigma^{\text {out }}$ via

$$
\Sigma^{\text {in }}=\left\{p=\left(A_{1}, B_{1}, \varepsilon_{1}, z_{1}\right) ;\left\langle e_{\mathrm{s}}^{*}, p-p_{\mathrm{s}}^{+}\right\rangle=\varepsilon_{1}=\delta\right\}, \quad \Sigma^{\text {out }}=\left\{\left(A_{1}, B_{1}, \varepsilon_{1}, z_{1}\right) ; A_{1}=\delta\right\},
$$

where we used the expression (3.6) for $e_{\mathrm{s}}^{*}$. Equation (3.7) guarantees that the unstable manifold of $p_{\mathrm{w}}^{+}$hits $\Sigma^{\mathrm{in}}$ at a point $p^{\text {in }}$ since its $\varepsilon_{1}$-component is never zero. Since the remaining stable 
eigenvalues produce much stronger decay, we see, upon using Shilnikov variables [2] and exploiting the expression

$$
\left\langle e_{\mathrm{s}}^{*}, p^{\text {in }}-p_{\mathrm{s}}^{+}\right\rangle=\varepsilon_{1}=\delta,
$$

that the $\left(\varepsilon_{1}, z_{1}\right)$-component of $p^{\text {in }}$ is given by

$$
\left(\varepsilon_{1}^{\text {in }}, z_{1}^{\text {in }}\right)=\left(\delta, \sqrt{2}+\frac{\sqrt{2} \delta}{3}\left(\gamma_{0}^{2}+\mathrm{o}_{\delta}(1)\right)\right),
$$

where $o_{\delta}(1)$ depends smoothly on $\gamma_{0}^{2}$. Using again Shilnikov variables [2], we obtain the estimates

$$
A_{1}^{\text {in }}=\delta \mathrm{e}^{-T / \sqrt{2}}\left[1+\mathrm{o}_{T}(1)\right], \quad z_{1}^{\text {out }}=\sqrt{2}+\frac{\sqrt{2} \delta}{3}\left(\gamma_{0}^{2}+\mathrm{o}_{\delta}(1)\right) \mathrm{e}^{-2 T / \sqrt{2}}\left[1+\mathrm{o}_{T}(1)\right]
$$

for the value of $A_{1}$ in $\Sigma^{\text {in }}$ and of $z_{1}$ in $\Sigma^{\text {out }}$, where $\mathrm{o}_{T}(1) \rightarrow 0$ as $T \rightarrow \infty$. Thus, we obtain

$$
z_{1}^{\text {out }}=\sqrt{2}+\frac{\sqrt{2}}{3 \delta}\left(\gamma_{0}^{2}+\mathrm{o}_{\delta}(1)\right)\left(A_{1}^{\text {in }}\right)^{2}\left[1+\mathrm{o}_{A_{1}^{\text {in }}}(1)\right] .
$$

Since $\varepsilon=\varepsilon_{1} A_{1}^{2}=\delta A_{1}^{2}$, and therefore $A_{1}^{2}=\varepsilon / \delta$, in $\Sigma^{\text {in }}$, and $z=z_{1} \delta$ in $\Sigma^{\text {out }}$, we get

$$
z^{\text {out }}=\sqrt{2}\left[\delta+\frac{\left(\gamma_{0}^{2}+\mathrm{o}_{\delta}(1)\right) \varepsilon}{3 \delta}\left[1+\mathrm{o}_{\varepsilon}(1)\right]\right],
$$

where $o_{\delta}(1)$ depends smoothly on $\gamma_{0}^{2}$. Using the scaling (3.3) with $\mu_{0}=1$, we finally obtain

$$
z^{\text {out }}=\sqrt{2}\left[\delta+\frac{\lambda+\mu \mathrm{o}_{\delta}(1)}{3 \delta}\left[1+\mathrm{o}_{\mu}(1)\right]\right]
$$

where $o_{\delta}(1)$ depends smoothly on $\lambda / \mu$. Thus, we see that $z^{\text {out }}$ is $C^{2}$ in $\gamma=\sqrt{\lambda}$ and $C^{1}$ in $\lambda$ in the region $|\lambda| \leq \mu$ that we considered here. In summary, we proved that we can define and continue the unstable subspace within the center space through the bifurcation point, at least for $|\lambda| \leq \mu$.

\section{Rescaling chart: Small parameters}

In the next step, we analyse (3.4) for small $\mu_{0}$ with $\left|\gamma_{0}\right|^{2}=1$. It is convenient to introduce the $\varepsilon$-directional blow-up chart whose coordinates are given by

$$
A_{2}=\frac{A}{\sqrt{\varepsilon}}, \quad B_{2}=\frac{B}{\varepsilon}, \quad \varepsilon_{2}=\varepsilon, \quad z_{2}=\frac{z}{\sqrt{\varepsilon}} .
$$

After factoring the Euler multiplier $\varepsilon$, equation (3.4) becomes

$$
\begin{aligned}
A_{2}^{\prime} & =B_{2} \\
B_{2}^{\prime} & =-\mu_{0} A_{2}+A_{2}^{3}+\mathrm{O}\left(\sqrt{\varepsilon_{2}}\right) \\
\varepsilon_{2}^{\prime} & =0 \\
z_{2}^{\prime} & =\gamma_{0}^{2}-\mu_{0}+3 A_{2}^{2}-z_{2}^{2} .
\end{aligned}
$$

We restrict our analysis to the region where $\mu_{0} \geq 0,\left|\gamma_{0}\right|^{2}=1$ and $\operatorname{Re} \gamma_{0} \geq 0$ which corresponds to the spectral region $\lambda \in \mathbb{C} \backslash \mathbb{R}^{-}$where the cut is taken along the absolute spectrum $\mathbb{R}^{-}$. 
In this chart, the wave equilibria are given by

$$
p_{\mathrm{w}}=\left(\sqrt{\mu_{0}}, 0, \varepsilon_{2}, \sqrt{\gamma_{0}^{2}+2 \mu_{0}}\right)
$$

where the square root is assigned to ensure that $\operatorname{Re} z_{2} \geq 0$. For $\varepsilon=0$, the wave equilibria are connected to the singular equilibrium $p_{\mathrm{s}}^{+}$in the $A$-directional blow-up chart by the heteroclinic orbit

$$
A_{2}=-\sqrt{\mu_{0}} \operatorname{coth}\left(\sqrt{\frac{\mu_{0}}{2}} x\right), \quad B_{2}=\frac{1}{\sqrt{2}}\left(A_{2}^{2}-\mu_{0}\right)
$$

which exists provided $\mu_{0}>0$ but has a smooth limit as $\mu_{0} \rightarrow 0$ for each finite $x$. For $\varepsilon>0$ small and $\operatorname{Re} \gamma_{0}>0$, the one-dimensional weak unstable manifolds of the wave equilibria depend smoothly on $\varepsilon$ since the equilibria are normally hyperbolic for $\mu_{0}>0$ (the limit $\mu_{0}=0$ in the $(A, B)$ components has been considered in Section 2.1). As shown in [20, §3.3], the unstable manifold in the $z_{2}$-equation can then be continued smoothly up to $\operatorname{Re} \gamma_{0}=0$ using the variation-of-constants formula and the contraction-mapping principle in spaces of bounded functions with weight $1 /|x|$. Since the unstable manifold always has a nonzero component in the direction of $A_{2}$, we can change coordinates to the $A$-directional blow-up chart where $\varepsilon_{1}=\varepsilon / A^{2}=1 / A_{2}^{2}$ is bounded for the part of the unstable manifold outside a fixed neighborhood of the wave equilibrium. We monitor $\varepsilon_{1}$ along the heteroclinic until we hit the section $\Sigma^{\text {in }}$. The same computation as for $\mu_{0}=1$ shows that the expression (3.9) is still valid with uniform error estimate in $\left|\gamma_{0}\right|^{2}+\left|\mu_{0}\right|=1$ for $\mu_{0} \geq 0$.

Lastly, the case $\mu_{0}<0$ is very similar as we have to follow the unstable manifold of the wave equilibrium at $\mu_{0}=0$ to negative values of $\mu_{0}$. Since this can be achieved by utilizing the $\varepsilon$ directional blow-up chart as in the case $\mu_{0}>0$, we decided to omit the details.

\section{The Evans function near zero}

In summary, we demonstrated that the bundles $E^{\mathrm{u}}$ and $E^{\mathrm{s}}$ can be continued in the fashion claimed in Proposition 3.1, at least in a neighborhood of the asymptotic state $|A|=\delta$. To extend these bundles to $x=0$, where we shall match with $\operatorname{Fix}(\mathcal{R})$, we simply transport the bundles with the linearized flow. Since the bifurcating pulses are continuous in $\mu$, the linearized equation depends continuously on $\mu$ and $\gamma$, we see that the bundles, evaluated at $x=0$, depend on $\lambda$ and $\mu$ in the fashion stated in Proposition 3.1.

It remains to prove Proposition 3.2. We have to show that the derivative of $\mathcal{E}$, considered as a real-valued function of $\lambda \geq 0$, does not vanish. First, by Hypothesis 3 , there are no roots of the Evans function that correspond to even eigenfunctions. Thus, we can redefine the Evans function to measure only eigenvalues associated with odd eigenfunctions, which we may achieve by using the definition

$$
\mathcal{E}(\lambda)=\operatorname{det}\left[e_{1}^{\mathrm{u}}, \ldots, e_{n}^{\mathrm{u}}, e_{1}^{-\mathrm{R}}, \ldots, e_{n}^{-\mathrm{R}}\right]
$$

where $\left(e_{j}^{-\mathrm{R}}\right)_{j}$ is a basis of $\operatorname{Fix}(-\mathcal{R})$. Next, we notice that the null space of the matrix in the definition (3.10) of the Evans function is one-dimensional since the intersection of $E_{-}^{\mathrm{u}}$, evaluated at $x=0$, and $\operatorname{Fix}(-\mathcal{R})$ is one-dimensional and is not contained in the strong unstable subspace 
$E_{-}^{\text {uu }}$. Using the reduction to the center-bundle, we may calculate the Evans function in the section $|A|=\delta$ near the singular sphere. We obtain the expansion

$$
\mathcal{E}(\lambda)=z^{\text {out }}(\lambda)-z^{-R}(\lambda)+\mathrm{o}(\lambda)
$$

where $z^{-R}(\lambda)$ denotes the location of Fix $(-R)$ transported backwards from $x=0$ to the section. Now, we argue by contradiction and assume that $\mathcal{E}_{\lambda}(0)=0$. Upon differentiating (3.2) with respect to $\lambda$, we see that the derivative $z_{\lambda}^{\text {out }}(x)$ corresponds to a solution of the system

$$
\begin{aligned}
& u_{x}=v \\
& v_{x}=-D^{-1}\left[f_{u}(q(x) ; 0) u+q_{x}(x)\right]
\end{aligned}
$$

on $(-\infty, 0]$ and that $z_{\lambda}^{\text {out }}(0) \in \operatorname{Fix}(-R)$. Thus, to reach a contradiction to Hypothesis 4 , we need to show that the solution associated with $z_{\lambda}^{\text {out }}(x)$ is a generalized eigenfunction of $\lambda=0$ in $C_{\text {unif }}^{0}\left(\mathbb{R}, \mathbb{C}^{n}\right)$, i.e., that it is bounded as $x \rightarrow-\infty$. First, observe that

$$
z_{\lambda}^{\text {out }}=\left(\frac{b}{a}\right)_{\lambda}=\left(\frac{a_{x}}{a}\right)_{\lambda}=(\ln a)_{x \lambda} .
$$

On the other hand, using the results in this section and in Appendix B, it is not hard to see that

$$
\partial_{\lambda} z_{1}(y)=C_{1} \mathrm{e}^{-y / \sqrt{2}}\left[1+\mathrm{o}_{y}(1)\right]
$$

for some $C_{1} \neq 0$, where $\mathrm{o}_{y}(1) \rightarrow 0$ as $y \rightarrow-\infty$. Using the relation $\mathrm{d} y / \mathrm{d} x=|A|$, we see that this translates into $z_{\lambda}^{\text {out }}(x)=x\left[C_{2}+\mathrm{o}_{x}(1)\right]$ as $x \rightarrow-\infty$ for some constant $C_{2}$. Upon substituting this result into (3.12) and integrating over $x$, we obtain

$$
x^{2}\left[C_{3}+\mathrm{o}(1)\right]=(\ln a)_{\lambda}=\frac{a_{\lambda}}{a}
$$

for some constant $C_{3}$. Since $a(x)=q_{x}(x)=x^{-2}\left[C_{4}+\mathrm{o}_{x}(1)\right]$, we finally get $a_{\lambda}(x)=C_{5}+\mathrm{o}_{x}(1)$ as $x \rightarrow-\infty$ which shows that the solution of (3.11) that corresponds to $z_{\lambda}^{\text {out }}(x)$ indeed lies in $C_{\text {unif }}^{0}\left(\mathbb{R}, \mathbb{C}^{n}\right)$. As outlined above, this contradicts Hypothesis 4 and therefore proves Proposition 3.2.

\subsection{Turing bifurcations: Stability}

We prove Propositions 3.1 and 3.2 for Turing instabilities. Our strategy is similar to the one pursued in the preceding section where we treated pitchfork bifurcations.

First, we reduce the eigenvalue problem in a neighborhood of the origin to an eight-dimensional center manifold. After carrying out the usual normal-form transformations on the center manifold, the vector field becomes (see [17] for details)

$$
\begin{aligned}
A_{x} & =B+\mathrm{i} A(1+P) \\
B_{x} & =-\mu A+|A|^{2} A+\mathrm{i} B(1+P)+A Q+R \\
a_{x} & =b+\mathrm{i} a+r_{1}(a, b, \bar{a}, \bar{b}, \lambda) \\
b_{x} & =(\lambda-\mu) a+2|A|^{2} a+A^{2} \bar{a}+r_{2}(a, b, \bar{a}, \bar{b}, \lambda) \\
\bar{a}_{x} & =\bar{b}+\mathrm{i} \bar{a}+r_{3}(a, b, \bar{a}, \bar{b}, \lambda) \\
\bar{b}_{x} & =(\lambda-\mu) \bar{a}+2|A|^{2} \bar{a}+A^{2} a+r_{4}(a, b, \bar{a}, \bar{b}, \lambda)
\end{aligned}
$$


where $P, Q$ and $R$ have been introduced in (2.11), and the remainder terms $r_{j}$ account for higherorder terms. Note that $(\bar{a}, \bar{b})$ does not denote the complex conjugate of $(a, b)$. We scale $\lambda=\varepsilon \gamma_{0}^{2}$ and $\mu=\varepsilon \mu_{0}$, and use the $|A|$-directional blow-up variables

$$
A_{1}=\frac{A}{|A|}, \quad B_{1}=\frac{B}{|A|^{2}}, \quad \varepsilon_{1}=\frac{\varepsilon}{|A|^{2}}, \quad a_{1}=a, \quad b_{1}=\frac{b}{|A|}, \quad \bar{a}_{1}=\bar{a}, \quad \bar{b}_{1}=\frac{\bar{b}}{|A|} .
$$

After passing to a co-rotating frame

$$
\left(A_{1}, B_{1}, a_{1}, b_{1}, \bar{a}_{1}, \bar{b}_{1}\right) \longmapsto \mathrm{e}^{\mathrm{i} x}\left(A_{1}, B_{1}, a_{1}, b_{1}, \bar{a}_{1}, \bar{b}_{1}\right),
$$

introducing the additional fast angular variable $\psi=|A|^{2} \mathrm{e}^{\mathrm{i} x}$ and the invariant $N:=A_{1} \bar{B}_{1}$, and rescaling the independent variable to factor out the Euler multiplier $|A|$, we obtain the equation

$$
\begin{aligned}
\dot{A}_{1} & =B_{1}-A_{1} N_{\mathrm{r}}+\mathrm{O}(|A|) \\
\dot{B}_{1} & =\left(1-\mu_{0} \varepsilon_{1}\right) A_{1}-2 B_{1} N_{\mathrm{r}}+\mathrm{O}(|A|) \\
\dot{\varepsilon}_{1} & =-2 \varepsilon_{1} N_{\mathrm{r}} \\
|\dot{A}| & =|A| N_{\mathrm{r}} \\
\dot{\psi} & =|A|+\psi N_{\mathrm{r}} \\
\dot{a}_{1} & =b_{1}+\mathrm{O}(|A|) \\
\dot{b}_{1} & =\left(\gamma_{0}^{2}-\mu_{0}\right) \varepsilon_{1} a_{1}+2 a_{1}+A_{1}^{2} \bar{a}_{1}-N_{\mathrm{r}} b_{1}+\mathrm{O}(|A|) \\
\dot{\bar{a}}_{1} & =\bar{b}_{1}+\mathrm{O}(|A|) \\
\dot{\bar{b}}_{1} & =\left(\gamma_{0}^{2}-\mu_{0}\right) \varepsilon_{1} \bar{a}_{1}+2 \bar{a}_{1}+A_{1}^{2} a_{1}-N_{\mathrm{r}} \bar{b}_{1}+\mathrm{O}(|A|) .
\end{aligned}
$$

Note the first five equations coincide with (2.15). The higher-order terms in (3.13) respect the skew-product structure and linearity of the equations for $\left(a_{1}, b_{1}, \bar{a}_{1}, \bar{b}_{1}\right)$.

In the singular sphere $|A|=0$, the eigenvalue problem decouples into the two Sturm-Liouville eigenvalue problems

$$
\begin{aligned}
& \ddot{a}_{+}=\left[3+\left(\gamma_{0}^{2}-\mu_{0}\right) \varepsilon_{1}\right] a_{+}-N_{\mathrm{r}} \dot{a}_{+} \\
& \ddot{a}_{-}=\left[1+\left(\gamma_{0}^{2}-\mu_{0}\right) \varepsilon_{1}\right] a_{-}-N_{\mathrm{r}} \dot{a}_{-}
\end{aligned}
$$

for $a_{+}=a_{1}+\bar{a}_{1}$ and $a_{-}=a_{1}-\bar{a}_{1}$, where $\left(N_{\mathrm{r}}, \varepsilon_{1}\right)$ are evaluated at the singular heteroclinic orbit (3.7). Transforming to $z_{ \pm}=\dot{a}_{ \pm} / a_{ \pm}$and arguing as in (3.8), we see that neither of these eigenvalue problems has unstable spectrum in $\left|\arg \gamma_{0}\right|<\pi / 4+\delta$ for some small $\delta>0$.

As in the preceding section, we write the four linear equations for $\left(a_{1}, b_{1}, \bar{a}_{1}, \bar{b}_{1}\right)$ as a nonlinear system on the Grassmannian with coordinates $\left(b_{1}, \bar{b}_{1}\right)=Z_{1}\left(a_{1}, \bar{a}_{1}\right)$. The complex $2 \times 2$-matrix $Z_{1}$ satisfies the matrix Riccati equation

$$
\dot{Z}_{1}=M_{0}+M_{1} Z_{1}-Z_{1}^{2}
$$

where

$$
M_{0}=\left(\begin{array}{cc}
\left(\gamma_{0}^{2}-\mu_{0}\right) \varepsilon_{1}^{2}+2 & 1 \\
1 & \left(\gamma_{0}^{2}-\mu_{0}\right) \varepsilon_{1}^{2}+2
\end{array}\right), \quad M_{1}=\left(\begin{array}{cc}
-N_{\mathrm{r}} & 0 \\
0 & -N_{\mathrm{r}}
\end{array}\right)
$$


At the wave equilibrium $\left(B_{1}, \varepsilon_{1}\right)=(0,1)$, the $Z_{1}$-equation has a unique equilibrium with the property that it is stable inside the $Z_{1}$-equation. This equilibrium represents the unstable subspace of the linearization about the asymptotic Turing pattern. Within the $\left(A_{1}, B_{1}, \varepsilon_{1}\right)$-subspace, the wave equilibrium has a one-dimensional unstable manifold that points into the $|A|$-direction and that connects to the singular equilibrium $\left(A_{1}, B_{1}, \varepsilon_{1}\right)=(0,1 / \sqrt{2}, 0)$. The $Z_{1}$-equation evaluated at the singular equilibrium has a unique equilibrium

$$
Z_{\mathrm{s}}=\left(\begin{array}{ll}
3 & 1 \\
1 & 3
\end{array}\right)
$$

which is stable within the $Z_{1}$-equation with algebraically simple eigenvalues at $-3 / \sqrt{2}$ and $-5 / \sqrt{2}$, and a geometrically double eigenvalue at $-4 / \sqrt{2}$. In particular, as for pitchfork bifurcations, the leading eigenvector of the full linearization of (3.13) does not lie in the $Z_{1}$-subspace. We claim that the unstable manifold of the wave equilibrium in the full space connects to the stable singular equilibrium: Indeed, the flow on the Grassmannian is gradient-like, and convergence to an unstable equilibrium would generate an eigenvalue for the aforementioned Sturm-Liouville eigenvalue problems for $\operatorname{Re} a_{1}$ and $\operatorname{Im} a_{1}$ which we precluded, however.

We can now proceed as before and compute the asymptotics for the $Z_{1}$-equation at the point $|A|=\delta$ in terms of the value of $|A|$ at the wave equilibrium. The component $Z_{1}^{\text {out }}$ can be calculated from (3.14) using the expansion

$$
\varepsilon_{1}(y)=\varepsilon_{1}^{\text {in }} \mathrm{e}^{2 y / \sqrt{2}}\left[1+\mathrm{o}_{y}(1)\right]
$$

for $\varepsilon_{1}$ that follows from equation (B.8) in Appendix B. In particular, following the arguments presented in the preceding section, we have

$$
Z_{1}^{\text {out }}=Z_{1,0}^{\text {out }}+Z_{1,1}^{\text {out }} \gamma_{0}^{2} \mu_{0} \varepsilon_{1}+\mathrm{o}\left(\varepsilon_{1}\right)
$$

A similar analysis for the $\gamma$-rescaling chart completes the picture and shows that the bundle $E_{-}^{\mathrm{u}}$ can be extended as stated in Proposition 3.1.

It remains to outline the proof of Proposition 3.2. As in the case of pitchfork instabilities, we prove that $E_{-}^{\mathrm{u}}$ and $\operatorname{Fix}(-\mathcal{R})$ intersect along a one-dimensional subspace that is generated by the derivative of the pulse. In the cross-section $|A|=\delta$ on the center-bundle, this corresponds to an at least one-dimensional null space of $Z^{\text {out }}-Z^{-R}$ where $Z^{-R}$ is the point on the Grassmannian that corresponds to the intersection of $\operatorname{Fix}(-\mathcal{R})$ with the four-dimensional center bundle, transported to $|A|=\delta$. If the dimension of the intersection were larger than one, the dimension of the null space of $\mathcal{L}_{*}$ would be at least two in contradiction to Hypothesis 4 . The projection of the derivative with respect to $\lambda$ of the linearized equation onto the one-dimensional null space of $Z^{\text {out }}-Z^{-R}$ is zero if, and only if, there exists a pre-image of $q^{\prime}$ in $L^{2}$. This finishes the proof of Proposition 3.2.

\section{Stability for oscillatory bifurcations}

We shall prove Theorem 6 for the symmetric defect $q_{0}(x ; \mu)$. Since the spectrum of the asymptotic wave trains is inherited from the Ginzburg-Landau equation, it is marginally stable on account 
of Hypothesis 10. We therefore concentrate on point spectrum near the Floquet exponent $\lambda=0$. We remark that [21, Theorem 6.1] together with our existence proof implies that the symmetric (and the asymmetric) pulse will have two Floquet exponents at zero, which are generated by the invariance with respect to translations in space and time. This is a peculiar situation as the primary pulse had only a single eigenvalue (at the origin). We shall see that the Nozaki-Bekki holes will contribute two Floquet exponents to the spectrum of the modulated pulses, yielding a total of three Floquet exponents close to zero. As argued above, two of these exponents are at the origin. As we shall see, the remaining eigenvalue is associated with a change of the relative phase of the asymptotic wave trains to the left and right of the pulse.

Floquet exponents of the symmetric pulse are determined by the existence of bounded nonzero solutions $u(x, t)$ to

$$
\omega_{0}(\mu) u_{\tau}=D u_{x x}+\left[f_{u}\left(q_{0}\left(x, \tau / \omega_{0}(\mu) ; \mu\right) ; \mu\right)-\lambda\right] u, \quad x \in \mathbb{R}
$$

that have temporal period $2 \pi$. We write this equation as

$$
\begin{aligned}
& u_{x}=v \\
& v_{x}=D^{-1}\left[\omega_{0}(\mu) \partial_{\tau}-f_{u}\left(q_{0}\left(x, \tau / \omega_{0}(\mu) ; \mu\right) ; \mu\right)+\lambda\right] u
\end{aligned}
$$

where $(u, v)(x) \in Y=H^{1 / 2}\left(S^{1}, \mathbb{C}^{2 n}\right) \times L^{2}\left(S^{1}, \mathbb{C}^{2 n}\right)$ for each fixed $x$. We will often write the modulated-wave equation (2.19) in the form

$$
U_{x}=\mathcal{A}_{0} U+\mathcal{F}(U ; \mu), \quad U=(u, v) \in Y
$$

and use the notation $Q_{0}(x ; \mu)=\left(q_{0}, \partial_{x} q_{0}\right)\left(x, \tau / \omega_{0}(\mu) ; \mu\right) \in Y$ for the modulated pulse. With the obvious notation, (4.2) can then be written as

$$
U_{x}=\left[\mathcal{A}_{0}+\mathcal{F}_{U}\left(Q_{0}(x ; \mu) ; \mu\right)+\lambda \mathcal{B}\right] U
$$

Since the modulated pulse $q_{0}(x, t ; \mu)$ is symmetric with respect to the reverser $\mathcal{R}_{0}$, it suffices to find bounded nonzero solutions $(u, v)(x, \tau)$ of $(2.21)$ for $x \in \mathbb{R}^{-}$that satisfy the boundary condition

$$
(u, v)(0, \cdot) \in \operatorname{Fix}\left(\mathcal{R}_{0}\right)
$$

or

$$
(u, v)(0, \cdot) \in \operatorname{Fix}\left(-\mathcal{R}_{0}\right)
$$

We will focus on (4.5) as it is the more complicated case.

Since the modulated pulse $q_{0}(x, t ; \mu)$ is constructed by gluing together the standing pulse $q(x)$ and the Nozaki-Bekki holes $A_{\mathrm{nb}}(X)$, we will construct solutions to (4.2) by gluing together eigenfunctions of the linearization of (1.1) about the standing pulse and of the complex Ginzburg-Landau equation linearized about the Nozaki-Bekki holes (see [14] for similar ideas). 


\subsection{The linearization near the origin}

We again reduce the modulated-wave equation (2.19) and its linearization (4.2) simultaneously to the center-unstable and the eight-dimensional center manifold. We also straighten out the strong unstable fibers inside the center-unstable manifold. As a consequence, the vector field on the center manifold decouples from the flow on the unstable fibers, and the vector field corresponding to (4.2) inside the center-unstable manifold is therefore of skew-product form. In particular, the linearization on the center manifold about the modulated wave decoules and is, in fact, given by the linearization about the projection of the modulated pulse onto the center manifold along the unstable foliation. Using the scaling

$$
a=A, \quad b=\sqrt{\mu} B, \quad \bar{a}=\bar{A}, \quad \bar{b}=\sqrt{\mu} \bar{B}, \quad x=\frac{X}{\sqrt{\mu}}, \quad \lambda=\mu \check{\lambda},
$$

this linearization is given by

$$
\begin{aligned}
A_{x}= & B+\mathrm{O}(|\mu|(|A|+|B|+|\bar{A}|+|\bar{B}|)) \\
B_{x}= & \frac{1}{1+\mathrm{i} \alpha}\left[-(1+\mathrm{i} \Omega) A+2(1+\mathrm{i} \beta)\left|A_{\mathrm{nb}}(X ; \mu)\right|^{2} A+(1+\mathrm{i} \beta) A_{\mathrm{nb}}(X ; \mu)^{2} \bar{A}+\check{\lambda} A\right] \\
& +\mathrm{O}(|\mu|(|A|+|B|+|\bar{A}|+|\bar{B}|)) \\
\bar{A}_{x}= & \bar{B}+\mathrm{O}(|\mu|(|A|+|B|+|\bar{A}|+|\bar{B}|)) \\
\bar{B}_{x}= & \frac{1}{1-\mathrm{i} \alpha}\left[-(1-\mathrm{i} \Omega) \bar{A}+2(1-\mathrm{i} \beta)\left|A_{\mathrm{nb}}(X ; \mu)\right|^{2} \bar{A}+(1-\mathrm{i} \beta) \bar{A}_{\mathrm{nb}}(X ; \mu)^{2} A+\check{\lambda} \bar{A}\right] \\
& +\mathrm{O}(|\mu|(|A|+|B|+|\bar{A}|+|\bar{B}|))
\end{aligned}
$$

where the perturbed Nozaki-Bekki hole $A_{\mathrm{nb}}(X ; \mu)$ lies in the surface $\Gamma_{0}(\mu)$ for $X=0$. In particular, for $\mu=0,(4.7)$ is simply the linearization of the CGL about the standing Nozaki-Bekki hole. Note that $(\bar{A}, \bar{B})$ does not denote the complex conjugate of $(A, B)$.

For $\check{\lambda}=0$, the derivative $A_{\mathrm{nb}}^{\prime}(X ; \mu)$ and the phase-derivative $\mathrm{i} A_{\mathrm{nb}}(X ; \mu)$ are the unique nonzero bounded solutions of (4.7) on $\mathbb{R}^{-}$. For $\operatorname{Re} \check{\lambda}>0$, the linearization about the asymptotic wave trains has two unstable and two stable Floquet exponents. As proved in [21], there is a $\delta_{0}>0$ such that the $2: 2$ splitting with respect to the real parts of the Floquet exponents persists for all $\operatorname{Re} \check{\lambda} \geq-\delta_{0}$. We denote the associated linearly independent solutions of $(4.7)$ by $\phi_{j}(X ; \check{\lambda}, \mu)$ for $j=1,2$ with

$$
\begin{aligned}
& \phi_{1}(X ; 0, \mu)=\left(A_{\mathrm{nb}}^{\prime}(X ; \mu), B_{\mathrm{nb}}^{\prime}(X ; \mu), \bar{A}_{\mathrm{nb}}^{\prime}(X ; \mu), \bar{B}_{\mathrm{nb}}^{\prime}(X ; \mu)\right), \\
& \phi_{2}(X ; 0, \mu)=\left(\mathrm{i} A_{\mathrm{nb}}(X ; \mu), \mathrm{i} B_{\mathrm{nb}}(X ; \mu), \mathrm{i} \bar{A}_{\mathrm{nb}}(X ; \mu), \mathrm{i} \bar{B}_{\mathrm{nb}}(X ; \mu)\right),
\end{aligned}
$$

where $\left(\bar{A}_{\mathrm{nb}}, \bar{B}_{\mathrm{nb}}\right)$ denotes the complex conjugate of the perturbed Nozaki-Bekki hole. As $\mu \rightarrow 0$, these solutions converge to solutions of the CGL linearized about the standing Nozaki-Bekki hole for $\mu=0$.

In summary, the general bounded solution of (4.7) on $\mathbb{R}^{-}$is of the form

$$
(A, B, \bar{A}, \bar{B})(X)=\check{d}_{1} \phi_{1}(X ; \check{\lambda}, \mu)+\check{d}_{2} \phi_{2}(X ; \check{\lambda}, \mu)
$$

with $\breve{d}=\left(\breve{d}_{1}, \breve{d}_{2}\right) \in \mathbb{C}^{2}$. We shall later use the slightly more convenient coordinates

$$
(\operatorname{Re} A, \operatorname{Im} A, \operatorname{Re} B, \operatorname{Im} B):=\left(\frac{A+\bar{A}}{2}, \frac{A-\bar{A}}{2 \mathrm{i}}, \frac{B+\bar{B}}{2}, \frac{B-\bar{B}}{2 \mathrm{i}}\right) .
$$


Lastly, we remark that solutions with initial data in the strong unstable component $W$ will always decay exponentially to zero as $x \rightarrow-\infty$.

\subsection{The linearization near the standing pulse}

Using the estimate (2.28), we can write $(4.3)$ on $[-L, 0]$ as

$$
U_{x}=\left[\mathcal{A}_{0}+\mathcal{F}_{U}\left(q, q^{\prime} ; 0\right)+\lambda \mathcal{B}+\mathrm{O}(|\mu|)\right] U=:[\mathcal{A}(x)+\lambda \mathcal{B}+\mathrm{O}(|\mu|)] U
$$

with $U \in Y$, where $q$ denotes the standing pulse at $\mu=0$. We seek solutions $U(x)$ of (4.11) that satisfy

$$
U(0) \in \operatorname{Fix}\left(-\mathcal{R}_{0}\right) .
$$

We will choose $L$ later but emphasize that it will depend only on properties of $(4.11)$ at $(\mu, \lambda)=0$ : in particular, its choice depends only on properties of the primary pulse.

First, we set $(\mu, \lambda)=0$. Equation (4.11) is then simply the linearization

$$
U_{x}=\mathcal{A}(x) U
$$

of the modulated-wave equation (2.19) about the standing pulse $Q(x)=\left(q, q^{\prime}\right)$. Note that (4.13) leaves the spaces $Y_{\ell}$ from $\S 2.3$ invariant, and recall also that $Q$ decays to zero exponentially.

The action of $\mathcal{R}_{0}$ on $Y_{\ell}$ has been determined in (2.20): we see that $-\mathcal{R}_{0}$ acts like $\mathcal{R}_{\pi}$ on each $Y_{\ell}$ with $\ell$ odd. Specifically, $-\mathcal{R}_{0}$ acts as $\mathcal{R}_{\pi}$ on $Y_{1}$. It follows that the center-unstable manifold $W^{\mathrm{cu}}(0)$ of the origin intersects $\operatorname{Fix}\left(-\mathcal{R}_{0}\right)$ in a three-dimensional subspace spanned by the derivative $Q^{\prime}(x)$ of the pulse $Q=\left(q, q^{\prime}\right)$ in $Y_{0}$ and two solutions $R_{j}(x)$ with $j=1,2$ in $Y_{1}$ such that $R_{1}(-L)$ and $R_{2}(-L)$ are an orthogonal basis of the tangent space of the surface $\Gamma_{\pi}(0)$ at the origin (see again $\S 2.3)$. In particular, $R_{1}(-L)$ and $R_{2}(-L)$ lie in the center component. Note that the solutions $R_{1}(x)$ and $R_{2}(x)$ grow linearly in $x$ for large $x$ due to (2.25) and (2.26). There are two other, linearly independent solutions, denoted by $V_{0}^{\mathrm{c}}(x)$, that lie asymptotically in the four-dimensional center space of the linearization about $U=0$ : Since $Q$ decays to zero exponentially, these solutions can be chosen to have $B=0$ for all large $x$ once we transform into the coordinates $(A, B, W)$ from the preceding section.

In summary, we have

$$
\begin{aligned}
\operatorname{Fix}\left(-\mathcal{R}_{0}\right) \cap T_{Q(0)} W^{\mathrm{cu}}(0) & =\operatorname{span}\left\{Q^{\prime}(0), R_{1}(0), R_{2}(0)\right\} \\
{\left[\operatorname{Fix}\left(-\mathcal{R}_{0}\right)+T_{Q(0)} W^{\mathrm{cu}}(0)\right]^{\perp} } & =\operatorname{span}\left\{Q^{*}(0)\right\},
\end{aligned}
$$

and we can find subspaces $Y_{\text {rev }}$ of $\operatorname{Fix}\left(-\mathcal{R}_{0}\right)$ and $Y_{\mathrm{u}}$ of $T_{Q(0)} W^{\mathrm{u}}(0)$ so that

$$
\operatorname{span}\left\{Q^{\prime}(0), R_{1}(0), R_{2}(0)\right\} \oplus \operatorname{span}\left\{Q^{*}(0)\right\} \oplus Y_{\text {rev }} \oplus Y_{\mathrm{u}} \oplus Y_{\mathrm{c}}=Y .
$$

Here, $Y_{\mathrm{c}}=\operatorname{span}\left\{Y_{0}^{\mathrm{c}}(0)\right\}$, while $Q^{*}(x)$ denotes the unique (up to constant multiples) bounded nonzero solution of the adjoint variational equation about the standing pulse in $Y_{0}$. Lastly, we denote the center stable, strong unstable, and strong stable evolutions of (4.13) by $\Phi^{\mathrm{cs}}(x, y), \Phi^{\mathrm{u}}(x, y)$, 
and $\Phi^{\mathrm{s}}(x, y)$, respectively. In particular, there are constants $C>0$ and $\eta>0$ such that

$$
\left\|\Phi^{\mathrm{cs}}(y, x)\right\|_{L(Y)} \leq C|x-y|, \quad\left\|\Phi^{\mathrm{u}}(x, y)\right\|_{L(Y)} \leq C \mathrm{e}^{\eta(x-y)}, \quad\left\|\Phi^{\mathrm{s}}(y, x)\right\|_{L(Y)} \leq C \mathrm{e}^{-\eta(y-x)}
$$

for $x \leq y \leq 0$. Throughout the rest of this section, various different constants, which are all independent of $L, \mu$ and $\lambda$, are denoted by $C$.

We make the ansatz

$$
U(x)=d_{0} Q^{\prime}(x)+\sqrt{\mu}\left(d_{1} R_{1}(x)+d_{2} R_{2}(x)\right)+V(x)=: R(x) d+V(x)
$$

with $d=\left(d_{0}, d_{1}, d_{2}\right) \in \mathbb{C}^{3}$ and remark that

$$
\|R d\|:=\sup _{x \in[-L, 0]}|R(x) d|_{Y} \leq C\left(\left|d_{0}\right|+\sqrt{\mu} L\left(\left|d_{1}\right|+\left|d_{2}\right|\right)\right) .
$$

Any bounded solution $U(x)$ of $(4.13)$ on $(-L, 0)$ for $\mu>0$ can now be obtained via a solution $V$ to the fixed-point equation

$$
\begin{aligned}
V(x)= & \Phi^{\mathrm{s}}(x,-L) V_{0}^{\mathrm{s}}+\Phi^{\mathrm{u}}(x, 0) V_{0}^{\mathrm{u}}+V_{0}^{\mathrm{c}}(x) \\
& +\int_{0}^{x} \Phi^{\mathrm{u}}(x, y)[\lambda \mathcal{B}+\mathrm{O}(\mu)](V(y)+R(y) d) \mathrm{d} y \\
& +\int_{-L}^{x} \Phi^{\mathrm{cs}}(x, y)[\lambda \mathcal{B}+\mathrm{O}(\mu)](V(y)+R(y) d) \mathrm{d} y
\end{aligned}
$$

on $C^{0}([-L, 0], Y)$, where $V_{0}^{\mathrm{u}} \in Y_{\mathrm{u}}$. Using the estimate (4.16), we obtain the estimate

$$
\text { r.h.s. of }(4.18) \leq C\left(\left|V_{0}^{\mathrm{c}}\right|+\left|V_{0}^{\mathrm{s}}\right|+\left|V_{0}^{\mathrm{u}}\right|+L^{2}(|\lambda|+\mu \mid)(\|V\|+\|R d\|)\right)
$$

for the right-hand side of (4.18), where $\|V\|=\sup _{x \in[-L, 0]}|V(x)|_{Y}$. Thus, choosing $\mu$ and $\lambda$ small compared with $L$, we can solve (4.18) uniquely for $V$ using the contraction-mapping principle. The solution $V(x)$ satisfies

$$
\begin{aligned}
\|V\| \leq & C\left(\left|V_{0}^{\mathrm{c}}\right|+\left|V_{0}^{\mathrm{s}}\right|+\left|V_{0}^{\mathrm{u}}\right|+L(|\lambda|+|\mu|)\|R d\|\right) \\
V(0)= & \Phi^{\mathrm{s}}(0,-L) V_{0}^{\mathrm{s}}+V_{0}^{\mathrm{u}}+V_{0}^{\mathrm{c}}(0)+\int_{-L}^{0} \Phi^{\mathrm{cs}}(0, y)[\lambda \mathcal{B}+\mathrm{O}(\mu)](V(y)+R(y) d) \mathrm{d} y \\
= & V_{0}^{\mathrm{u}}+V_{0}^{\mathrm{c}}(0)+\int_{-L}^{0} \Phi^{\mathrm{cs}}(0, x)[\lambda \mathcal{B}+\mathrm{O}(\mu)] R(x) d \mathrm{~d} x \\
& +\mathrm{O}\left(\mathrm{e}^{-\eta L}\left|V_{0}^{\mathrm{s}}\right|+L^{2}(|\lambda|+|\mu|)\left(\left|V_{0}^{\mathrm{u}}\right|+\left|V_{0}^{\mathrm{c}}\right|+\left|V_{0}^{\mathrm{s}}\right|\right)+L^{4}(|\lambda|+|\mu|)^{2}\|R d\|\right) \\
V(-L)= & V_{0}^{\mathrm{s}}+\Phi^{\mathrm{u}}(-L, 0) V_{0}^{\mathrm{u}}+V_{0}^{\mathrm{c}}(-L)+\int_{0}^{-L} \Phi^{\mathrm{u}}(-L, y)[\lambda \mathcal{B}+\mathrm{O}(\mu)](V(y)+R(y) d) \mathrm{d} y \\
= & V_{0}^{\mathrm{s}}+V_{0}^{\mathrm{c}}(-L)+\mathrm{O}\left(\mathrm{e}^{-\eta L}\left|V_{0}^{\mathrm{u}}\right|+(|\lambda|+|\mu|)\left(\left|V_{0}^{\mathrm{u}}\right|+\left|V_{0}^{\mathrm{c}}\right|+\left|V_{0}^{\mathrm{s}}\right|+\|R d\|\right)\right) .
\end{aligned}
$$

\subsection{Matching}

First, we need to ensure that $U(0) \in \operatorname{Fix}\left(-\mathcal{R}_{0}\right)$ where

$$
\begin{aligned}
U(0)= & R(0) d+V(0) \\
= & d_{0} Q^{\prime}(0)+\sqrt{\mu}\left(d_{1} R_{1}(0)+d_{2} R_{2}(0)\right)+V_{0}^{\mathrm{u}}+V_{0}^{\mathrm{c}}(0)+\int_{-L}^{0} \Phi^{\mathrm{cs}}(0, x)[\lambda \mathcal{B}+\mathrm{O}(\mu)] R(x) d \mathrm{~d} x \\
& +\mathrm{O}\left(\mathrm{e}^{-\eta L}\left|V_{0}^{\mathrm{s}}\right|+L^{2}(|\lambda|+|\mu|)\left(\left|V_{0}^{\mathrm{u}}\right|+\left|V_{0}^{\mathrm{c}}\right|+\left|V_{0}^{\mathrm{s}}\right|\right)+L^{4}(|\lambda|+|\mu|)^{2}\|R d\|\right) .
\end{aligned}
$$


We see that there are unique elements $\left(V_{0}^{\mathrm{u}}, V_{0}^{\mathrm{c}}(0)\right) \in Y_{\mathrm{u}} \times Y_{\mathrm{c}}$ so that the components of $U(0)$ in $Y_{\mathrm{u}} \times Y_{\mathrm{c}}$ are zero. The elements $Y_{0}^{\mathrm{u}}$ and $Y_{0}^{\mathrm{c}}(0)$ are linear in $\left(Y_{0}^{\mathrm{s}}, d\right)$ and satisfy

$$
\left|Y_{0}^{\mathrm{u}}\right|+\left|Y_{0}^{\mathrm{c}}\right| \leq C\left(\left(\mathrm{e}^{-\eta L}+L^{2}(|\lambda|+|\mu|)\right)\left|V_{0}^{\mathrm{s}}\right|+L^{2}(|\lambda|+|\mu|)\|R d\|\right)
$$

since $R(0) \in \operatorname{Fix}\left(-\mathcal{R}_{0}\right)$. Using (4.14), (4.15) and $R(0) \in \operatorname{Fix}\left(-\mathcal{R}_{0}\right)$, we find that $U(0) \in \operatorname{Fix}\left(-\mathcal{R}_{0}\right)$ is equivalent to solving

$$
\int_{-L}^{0}\left\langle Q^{*}(x),[\lambda \mathcal{B}+\mathrm{O}(\mu)] R(x) d\right\rangle \mathrm{d} x+\mathrm{O}\left(\left(\mathrm{e}^{-\eta L}+L^{2}(|\lambda|+|\mu|)\right)\left|V_{0}^{\mathrm{s}}\right|+L^{4}(|\lambda|+|\mu|)^{2}\|R d\|\right)=0 .
$$

Before solving (4.20), we shall match near the origin. We first make sure that $U(-L)$ lies in the tangent space of the center-unstable manifold. Afterwards, we match the center part of $U(-L)$ to the solutions (4.9).

Using the expression for $V(-L)$ in (4.19) together with the fact that $R(-L)$ lies in the tangent space of the center-unstable manifold, we obtain that $U(-L)$ is in the tangent space of the centerunstable manifold provided we set $V_{0}^{\mathrm{s}}=0$. Thus, we have solved uniquely for $\left(V_{0}^{\mathrm{u}}, V_{0}^{\mathrm{c}}, V_{0}^{\mathrm{s}}\right)$ which satisfy the estimate

$$
\left|V_{0}^{\mathrm{u}}\right|+\left|V_{0}^{\mathrm{c}}\right| \leq C L(|\lambda|+|\mu|)\left(\mid d_{0}+\sqrt{\mu} L\left(\left|d_{1}\right|+\left|d_{2}\right|\right)\right), \quad V_{0}^{\mathrm{s}}=0
$$

At this point, we should say precisely how we choose $L$. We note that $[15,(4.3)]$ and the fact that the eigenvalue $\lambda=0$ of the primary pulse $Q(x)$ is simple imply that

$$
\int_{-\infty}^{0}\left\langle Q^{*}(x), \mathcal{B} Q^{\prime}(x)\right\rangle \mathrm{d} x \neq 0 .
$$

We then define

$$
M_{0}:=\int_{-L}^{0}\left\langle Q^{*}(x), \mathcal{B} Q^{\prime}(x)\right\rangle \mathrm{d} x
$$

which is $\mathrm{O}\left(\mathrm{e}^{-\eta L}\right)$ close to the nonzero integral at $L=\infty$. Thus, we choose $L \gg 1$ so large that $M_{0} \neq 0$ and so that the preceding calculations work out for $(\mu, \lambda)=0$, which requires that the eror terms $\mathrm{O}\left(\mathrm{e}^{-\eta L}\right)$ are small enough. This choice of $L$ is fixed from now on, and $(\mu, \lambda)$ are chosen small compared to $L$.

The preceding analysis shows that the condition (4.12) is equivalent to (4.20) which, upon substituting the estimate (4.21), becomes

$$
M_{0} \lambda d_{0}+\mathrm{O}\left(\left(|\mu|+|\lambda|^{2}\right)\left|d_{0}\right|+(|\lambda|+\sqrt{\mu})(|\lambda|+|\mu|)\left(\left|d_{1}\right|+\left|d_{2}\right|\right)\right)=0 .
$$

On the other hand, we have shown that $U(-L)$ is in the tangent space of the center-unstable manifold, and its center component is given by

$$
U^{\mathrm{c}}(-L)=V_{0}^{\mathrm{c}}(-L)+\sqrt{\mu}\left(d_{1} R_{1}(-L)+d_{2} R_{2}(-L)\right) .
$$

We need to match $U^{\mathrm{c}}(-L)$ with the general bounded solution (4.10) of the center part of the linearization. Since (4.10) is expressed in scaled variables, we apply the scaling (4.6) also to $U^{\mathrm{c}}(-L)$. 
Using the estimate (4.21) together with the fact that the $B$-component of $V_{0}^{\mathrm{c}}(-L)$ vanishes, we obtain

$$
U^{\mathrm{c}}(-L) \longmapsto d_{1} r_{1}(-L)+d_{2} r_{2}(-L)+\mathrm{O}(\mu)\left|d_{0}\right|+\mathrm{O}\left(\mu^{3 / 2}\right)\left(\left|d_{1}\right|+\left|d_{2}\right|\right)
$$

where, in the $(\operatorname{Re} A, \operatorname{Im} A, \operatorname{Re} B, \operatorname{Im} B)$ coordinates,

$$
r_{1}(-L)=\left(\sqrt{\mu} \operatorname{Re} \rho_{\pi}(0), \sqrt{\mu} \operatorname{Im} \rho_{\pi}(0), 1,0\right), \quad r_{2}(-L)=\left(-\sqrt{\mu} \operatorname{Im} \rho_{\pi}(0), \sqrt{\mu} \operatorname{Re} \rho_{\pi}(0), 0,1\right)
$$

since $R_{j}(-L) \in \Gamma_{\pi}(0)$. The resulting matching condition is given by

$$
d_{1} r_{1}(-L)+d_{2} r_{2}(-L)+\mathrm{O}(\mu)\left|d_{0}\right|+\mathrm{O}\left(\mu^{3 / 2}\right)\left(\left|d_{1}\right|+\left|d_{2}\right|\right)=\check{d}_{1} \phi_{1}(X ; \check{\lambda}, \mu)+\check{d}_{2} \phi_{2}(X ; \check{\lambda}, \mu) .
$$

\subsection{Sharper estimates}

It will be convenient to revise our ansatz slightly. Instead of using $d_{0} Q^{\prime}(x)$, we shall use the derivative $d_{0} Q_{0}^{\prime}(x ; \mu)$ of the modulated pulse $Q_{0}(x ; \mu)$. Note that $\left\|Q^{\prime}-Q_{0}^{\prime}(x ; \mu)\right\|=\mathrm{O}(\mu)$ on $[-L, 0]$ by $(2.28)$. Since we still have $R(0) \in \operatorname{Fix}\left(-\mathcal{R}_{0}\right)$, the arguments above are still valid, except that the center component of $U(-L)$ now contains a contribution from the center part of $Q_{0}^{\prime}(-L ; \mu)$. Transforming the expression (2.27) using the scaling (4.6), we see that the center part of $Q_{0}^{\prime}(-L ; \mu)$ is $\mathrm{O}(\mu)$ in the coordinates $(A, B)$. Thus, the error estimates in (4.25) remain true. On the other hand, $Q_{0}^{\prime}(x ; \mu)$ is an exact solution at $\lambda=0$ of the $\mu$-dependent linearization. Therefore, the $\mathrm{O}(\mu) d_{0}$ term in (4.23) becomes $\mathrm{O}\left(\mu^{2}\right) d_{0}$. In addition, we need to replace $M_{0}$ by $M_{0}+\mathrm{O}(\mu)$, since we need to evaluate the right-hand side of $(4.22)$ at $Q_{0}^{\prime}(x ; \mu)$ instead of $Q^{\prime}(x)$. Thus, in summary, equation (4.23) is replaced by

$$
\left(M_{0}+\mathrm{O}(|\mu|)\right) \lambda d_{0}+\mathrm{O}\left(\left(|\mu|^{2}+\lambda^{2}\right)\left|d_{0}\right|+(|\lambda|+\sqrt{\mu})(|\lambda|+|\mu|)\left(\left|d_{1}\right|+\left|d_{2}\right|\right)\right)=0
$$

while (4.25) is unchanged:

$$
d_{1} r_{1}(-L)+d_{2} r_{2}(-L)+\mathrm{O}(\mu)\left|d_{0}\right|+\mathrm{O}\left(\mu^{3 / 2}\right)\left(\left|d_{1}\right|+\left|d_{2}\right|\right)=\check{d}_{1} \phi_{1}(X ; \check{\lambda}, \mu)+\check{d}_{2} \phi_{2}(X ; \check{\lambda}, \mu) .
$$

\subsection{The reduced eigenvalue problem}

It therefore remains to determine all $\lambda$ for which (4.26) and (4.27) have a nonzero solution $(d, \check{d})$. Since $(4.26)$ and $(4.27)$ are linear in $(d, \check{d})$, it suffices to seek roots of the associated determinant which is given by

$$
E(\check{\lambda}, \mu):=\left|\begin{array}{ccccc}
\mathrm{O}(\mu) & r_{1}(-L) & r_{2}(-L) & \phi_{1}(0 ; \check{\lambda}, \mu) & \phi_{2}(0 ; \check{\lambda}, \mu) \\
E_{0}(\mu \check{\lambda}, \mu) & \mathrm{O}\left(\mu^{3 / 2}\right) & \mathrm{O}\left(\mu^{3 / 2}\right) & 0 & 0
\end{array}\right|
$$

where the complex-valued function

$$
E_{0}(\lambda, \mu)=\left(M_{0}+\mathrm{O}(|\mu|)\right) \lambda+\mathrm{O}\left(|\mu|^{2}+|\lambda|^{2}\right)
$$

denotes the $d_{0}$-dependent term in (4.26). Since $E_{0}$ is smooth in its argument, we have

$$
E_{0}(\mu \check{\lambda}, \mu)=\mu\left[M_{0} \check{\lambda}+\mathrm{O}(|\mu|)\right]
$$


If we define

$$
E_{\mathrm{nb}}(\check{\lambda}, \mu):=\left|r_{1}(-L) r_{2}(-L) \phi_{1}(0 ; \check{\lambda}, \mu) \phi_{2}(0 ; \check{\lambda}, \mu)\right|,
$$

then the Evans function $E(\check{\lambda}, \mu)$ can be written as

$$
E(\check{\lambda}, \mu)=E_{0}(\mu \check{\lambda}, \mu) E_{\mathrm{nb}}(\check{\lambda}, \mu)+\mathrm{O}\left(\mu^{5 / 2}\right) .
$$

We already derived the expansion (4.28) for $E_{0}$ and focus therefore next on $E_{\mathrm{nb}}$. First, we emphasize that roots $\check{\lambda}$ of $E_{\mathrm{nb}}(\check{\lambda}, 0)$ are in one-to-one correspondence, counting multiplicity, with odd eigenvalues of the linearization of (1.8), posed in $C_{\text {odd }}^{0}$, about the Nozaki-Bekki holes $A_{\mathrm{nb}}$. Hypothesis 10 (ii) therefore implies that $E_{\mathrm{nb}}(\check{\lambda}, 0)$ has a simple root at $\check{\lambda}=0$ and no other roots in the closed right-half plane: in particular,

$$
M_{\mathrm{nb}}:=\partial_{\check{\lambda}} E_{\mathrm{nb}}(0,0) \neq 0 .
$$

On the other hand, we can compute $E_{\mathrm{nb}}(0, \mu)$ as follows: We substitute (4.24) for $r_{j}(-L)$ and (4.9) for $\phi_{j}$ at $\check{\lambda}=0$. Next, we note that $\left(A_{\mathrm{nb}}, B_{\mathrm{nb}}\right)(0 ; \mu) \in \Gamma_{0}(\mu)$, where $\Gamma_{0}(0)$ is given in $(2.26)$. Thus, we obtain

$$
\begin{aligned}
E_{\mathrm{nb}}(0, \mu) & =\left|\begin{array}{cccc}
\sqrt{\mu} \operatorname{Re} \rho_{\pi}(0) & -\sqrt{\mu} \operatorname{Im} \rho_{\pi}(0) & 1 & -\sqrt{\mu} \operatorname{Im} \rho_{0}(0)+\mathrm{O}(\mu) \\
\sqrt{\mu} \operatorname{Im} \rho_{\pi}(0) & \sqrt{\mu} \operatorname{Re} \rho_{\pi}(0) & \mathrm{O}(\sqrt{\mu}) & \sqrt{\mu} \operatorname{Re} \rho_{0}(0)+\mathrm{O}(\mu) \\
1 & 0 & \mathrm{O}(\sqrt{\mu}) & 0 \\
0 & 1 & \mathrm{O}(\sqrt{\mu}) & 1+\mathrm{O}(\mu) \\
& =\sqrt{\mu} \operatorname{Re}\left(\rho_{0}(0)-\rho_{\pi}(0)\right)+\mathrm{O}(\mu)
\end{array}\right|
\end{aligned}
$$

from which we conclude that

$$
E_{\mathrm{nb}}(\check{\lambda}, \mu)=M_{\mathrm{nb}} \check{\lambda}+\sqrt{\mu} \operatorname{Re}\left(\rho_{0}(0)-\rho_{\pi}(0)\right)+\mathrm{O}\left(\sqrt{\mu}|\check{\lambda}|+|\mu|+|\check{\lambda}|^{2}\right)
$$

$\operatorname{near}(\check{\lambda}, \mu)=0$ with $M_{\mathrm{nb}} \neq 0$.

Substituting the expansions (4.28) and (4.30) into the equation

$$
E_{0}(\mu \check{\lambda}, \mu) E_{\mathrm{nb}}(\check{\lambda}, \mu)+\mathrm{O}\left(\mu^{5 / 2}\right)=0
$$

and dividing by $\mu$, we obtain the reduced equation

$$
\left[M_{0} \check{\lambda}+\mathrm{O}(\mu)\right]\left[M_{\mathrm{nb}} \check{\lambda}+\sqrt{\mu} \operatorname{Re}\left(\rho_{0}(0)-\rho_{\pi}(0)\right)+\mathrm{O}\left(\sqrt{\mu}|\check{\lambda}|+|\mu|+|\check{\lambda}|^{2}\right)\right]+\mathrm{O}\left(\mu^{3 / 2}\right)=0,
$$

where $M_{0}, M_{\mathrm{nb}} \neq 0$. Thus, we see that (4.31) has precisely two roots, given by

$$
\check{\lambda}=\mathrm{O}(\mu), \quad \check{\lambda}=\sqrt{\mu} \frac{\operatorname{Re}\left(\rho_{\pi}(0)-\rho_{0}(0)\right)}{M_{\mathrm{nb}}}+\mathrm{O}(\mu),
$$

in the region $\operatorname{Re} \check{\lambda}>-\delta$ for some $\delta>0$.

The scaling (4.6) allowed us to find all eigenvalues $\lambda$ of order $\mathrm{O}(\mu)$. To exclude small eigenvalues that do not obey this scaling, we use the rescaling

$$
\mu=\check{\mu}|\lambda|, \quad \lambda=\mathrm{e}^{\mathrm{i} \phi}|\lambda| .
$$

Proceeding as above, we recover (4.7) in the center manifold with $\check{\lambda}$ replaced by $\mathrm{e}^{\mathrm{i} \phi}$ and where the CGL is now linearized about the Nozaki-Bekki hole of size $\mathrm{O}(\check{\mu})$. Since the small Nozaki-Bekki holes do not have any $\mathrm{O}(1)$-eigenvalues with eigenfunctions that satisfy (4.5), we see that we already accounted for all eigenvalues. 


\subsection{The Floquet spectra of the symmetric and asymmetric source}

We computed the eigenvalues (4.32) belonging to eigenfunctions of the linearization about the symmetric modulated pulse that lie in $\operatorname{Fix}\left(-\mathcal{R}_{0}\right)$. The analysis for the eigenfunctions in $\operatorname{Fix}\left(-\mathcal{R}_{0}\right)$ is, in fact, easier since the only contribution comes from $E_{\mathrm{nb}}$ : we find a single eigenvalue $\check{\lambda}_{2}=\mathrm{O}(\mu)$. Thus, the Floquet spectrum near zero in the original eigenvalue parameter $\lambda=\sqrt{\mu} \check{\lambda}$ consists of three eigenvalues. We proved in [21, Theorem 6.1] that the linearization about a source has precisely two Floquet exponents at zero which are generated by spatial and temporal translations. The remaining Floquet exponent is given by

$$
\lambda_{0}=\Lambda \mu^{3 / 2}+\mathrm{O}\left(\mu^{2}\right), \quad \Lambda=\frac{\operatorname{Re}\left(\rho_{\pi}(0)-\rho_{0}(0)\right)}{M_{\mathrm{nb}}} .
$$

The Floquet spectrum of the asymmetric pulse looks the same except that the nonzero Floquet exponent $\lambda_{\pi}$ is given by

$$
\lambda_{\pi}=-\Lambda \mu^{3 / 2}+\mathrm{O}\left(\mu^{2}\right)=-\lambda_{0}+\mathrm{O}\left(\mu^{2}\right), \quad \Lambda=\frac{\operatorname{Re}\left(\rho_{\pi}(0)-\rho_{0}(0)\right)}{M_{\mathrm{nb}}} .
$$

In particular, if $\operatorname{Re} \rho_{0}(0) \neq \operatorname{Re} \rho_{\pi}(0)$, then one of the two pulses is spectrally stable, while the other is spectrally unstable. The dynamics created by the eigenvalues $\lambda_{0}$ and $\lambda_{\pi}$ is a relative phase shift of the wave trains to the left and right of the pulse: Thus, if the symmetric pulse is unstable, say, then we expect that solutions nearby will evolve towards the asymmetric pulse simply by changing the relative phase of the asymptotic wave trains to the left and right.

\section{Compatibility of our hypotheses}

In this section, we prove Theorem 7.

First, we argue that our hypotheses are robust: If they are satisfied for a specific reaction-diffusion system, then they are also true for all nearby systems (where we work in the $C^{k}$-topology for $k$ sufficiently large). Clearly, linear instabilities of the kind described in Hypotheses 1 and 2 for pitchforks, Hypotheses 5 and 6 for Turing instabilities, and in Hypotheses 7 and 8 for oscillatory instabilities are robust. Next, our blow-up constructions show that the unstable manifolds of the origin at onset depend smoothly on perturbations of the nonlinearity which guarantees robustness of Hypothesis 3, while standard perturbation theory for homoclinic orbits shows that Hypothesis 11 is robust. Similarly, the marginal-stability Hypotheses 4 and 12 are robust since the Evans function depends continuously on parameters. Lastly, Hypotheses 9 and 10 for the Nozaki-Bekki holes are transversality conditions which are again robust since the coefficients in the CGL equation depend smoothly on the data of the reaction-diffusion system.

It therefore remains to prove that the three classes are not empty. Our strategy is to take a reactiondiffusion system that admits a stable pulse and to couple it to another system that exhibits a linear pitchfork, Turing or Hopf instability. We shall omit the example for pitchfork bifurcations as it is virtually identical to the one for Turing instabilities; note that the equation

$$
u_{t}=u_{x x}+\mu u-u^{3}+u^{5}
$$


satisfies all hypotheses for pitchforks except that the linearization about the pulse has a single simple unstable eigenvalue.

\subsection{The reaction-diffusion system with a stable standing pulse}

We choose to work with the reaction-diffusion system

$$
\begin{aligned}
& \partial_{t} u_{1}=\partial_{x x} u_{1}-u_{1}+u_{1}^{3}+\frac{1}{2} u_{1} u_{2} \\
& \partial_{t} u_{2}=\frac{1}{\delta^{2}}\left[\frac{1}{\delta^{2}} \partial_{x x} u_{2}-12.5 \delta^{2} u_{2}-\frac{1}{2} u_{1}^{2}+c_{0} u_{1}^{2} u_{2}\right]
\end{aligned}
$$

that has been investigated recently in [3]. It has been proved in [3, Theorems 2.2 and 4.7] that there are positive constants $c_{0}, c_{1}>0$ such that (5.1) has, for each $0<\delta \ll 1$, a stable symmetric standing pulse $q(x)$ : We have $q(x) \rightarrow 0$ as $|x| \rightarrow \infty$, the spectrum of the linearization of (5.1) about $q(x)$ is contained in the open left-half plane with the exception of an algebraically simple eigenvalue at $\lambda=0$, and the first component of the pulse given by

$$
q_{1}(x)=\sqrt{2\left(1+c_{1}\right)} \operatorname{sech}\left(\sqrt{1+c_{1}} x\right)+\mathrm{O}(\delta)
$$

where the $\mathrm{O}(\delta)$ term is uniform in $x \in \mathbb{R}$ and valid in the $C^{2}$-topology. We fix $0<\delta \ll 1$ so small that $q_{1}(x)$ achieves its nondegenerate positive maximum $M$ at $x=0$.

\subsection{Turing instabilities: An example}

We shall prove that there is a function $g: \mathbb{R} \rightarrow \mathbb{R}$ and an $0<\varepsilon \ll 1$ such that the system

$$
\begin{aligned}
\partial_{t} u_{1} & =D_{1} \partial_{x x} u_{1}+f_{1}\left(u_{1}, u_{2}\right) \\
\partial_{t} u_{2} & =D_{2} \partial_{x x} u_{2}+f_{2}\left(u_{1}, u_{2}\right) \\
\partial_{t} v_{1} & =\partial_{x x} v_{1}-v_{1}+2 v_{2} \\
\partial_{t} v_{2} & =\frac{1}{6} \partial_{x x} v_{2}-\frac{1}{3} v_{1}+\frac{1}{2} v_{2}+\frac{1}{3} \mu v_{2}-\frac{1}{9} v_{2}^{3}-\frac{1}{3} \varepsilon g\left(u_{1}\right),
\end{aligned}
$$

with the first two equations given by (5.1), satisfies the assumptions of Theorems 3 and 4 .

First, we set $\varepsilon=0$ and linearize about $v=0$ to get

$$
L_{\mu}=\left(\begin{array}{cc}
\partial_{x x}-1 & 2 \\
-\frac{1}{3} & \frac{1}{6} \partial_{x x}+\frac{1}{2}+\frac{1}{3} \mu
\end{array}\right) .
$$

The spectrum of $L_{\mu}$ lies strictly to the left of the imaginary axis except for the critical dispersion relation given by

$$
\lambda(k)=-\frac{2}{5}(k-1)^{2}+\frac{2}{5} \mu+\mathrm{O}\left(|\mu|^{2}+|\mu||k-1|+|k-1|^{3}\right),
$$

which verifies Hypothesis 5. 
From now on, we set $\mu=0$ and consider the standing-wave equation

$$
\begin{aligned}
v_{1}^{\prime} & =v_{3} \\
v_{2}^{\prime} & =6 v_{4} \\
v_{3}^{\prime} & =v_{1}-2 v_{2} \\
v_{4}^{\prime} & =\frac{1}{3} v_{1}-\frac{1}{2} v_{2}+\frac{1}{9} v_{2}^{3}+\frac{1}{3} \varepsilon g\left(q_{1}(x)\right)
\end{aligned}
$$

associated with (5.2), where we already substituted the pulse of (5.1). First, we again set $\varepsilon=0$ in which case the four-dimensional center manifold of (5.3) is given simply by the equation for $v$. It is convenient to parametrize the center manifold using the complex coordinates $(A, B) \in \mathbb{C}^{2}$ given by

$$
\left(\begin{array}{l}
v_{1} \\
v_{2} \\
v_{3} \\
v_{4}
\end{array}\right)=\left(\begin{array}{rrrr}
1 & \mathrm{i} & 1 & -\mathrm{i} \\
1 & 0 & 1 & 0 \\
\mathrm{i} & 0 & -\mathrm{i} & 0 \\
\mathrm{i} & \frac{1}{6} & -\frac{\mathrm{i}}{6} & \frac{1}{6}
\end{array}\right)\left(\begin{array}{l}
A \\
B \\
\bar{A} \\
\bar{B}
\end{array}\right), \quad\left(\begin{array}{l}
A \\
B \\
\bar{A} \\
\bar{B}
\end{array}\right)=\frac{1}{2}\left(\begin{array}{rrrr}
0 & 1 & -\mathrm{i} & 0 \\
-\mathrm{i} & \mathrm{i} & -1 & 6 \\
0 & 1 & \mathrm{i} & 0 \\
\mathrm{i} & -\mathrm{i} & -1 & 6
\end{array}\right)\left(\begin{array}{l}
v_{1} \\
v_{2} \\
v_{3} \\
v_{4}
\end{array}\right) .
$$

Transforming (5.3) for $\varepsilon=0$ into these coordinates, and observing that the nonlinearity in (5.3) with $\varepsilon=0$ is cubic, we can find an additional coordinate transformation of the form "identity plus at least cubic terms in $(A, B)$ " to bring the vector field on the center manifold into normal form (up to order five, say). Since there are no quadratic terms present in the first place, this transformation does not change the coefficient of the $|A|^{2} A$ term which is easily seen to be one. Accounting now for the $\varepsilon g\left(u_{1}\right)$ term, we finally obtain the representation

$$
\begin{aligned}
& A_{x}=\mathrm{i} A+B+\mathrm{i} A P \\
& B_{x}=\mathrm{i} B+A|A|^{2}+\varepsilon g\left(q_{1}(x)\right)+\mathrm{i} B P+A Q+\mathrm{O}\left(|A|^{5}+|B|^{5}+(|A|+|B|)\left|\varepsilon g\left(q_{1}(x)\right)\right|\right)
\end{aligned}
$$

where $P$ and $Q$ are as in (2.11). In particular, we have established Hypothesis 6.

To verify the remaining Hypotheses 3 and 4, it suffices to prove that the unstable manifold of the origin intersects $\operatorname{Fix}(\mathcal{R})$ transversely at the homoclinic orbit. In the coordinates (5.4), the reverser $\mathcal{R}$ of (5.3) becomes

$$
\mathcal{R}: \quad(A, B) \longmapsto(\bar{A},-\bar{B})
$$

(see [25, Lemma 2.11]) with fixed-point space

$$
\operatorname{Fix}(\mathcal{R})=\{(A, B)=(a, \mathrm{i} b) ; a, b \in \mathbb{R}\} .
$$

For $\varepsilon=0$, we have the expansion (2.18):

$$
W^{\mathrm{u}}(0)=\left\{(A, B)=\left(r \mathrm{e}^{\mathrm{i} \phi}, \frac{r^{2} \mathrm{e}^{\mathrm{i} \phi}}{\sqrt{2}}\right)+\mathrm{O}\left(r^{3}\right) ;|r| \ll 1\right\} .
$$

In particular, the pulse $(u, v)=(q, 0)$ lies in both $\operatorname{Fix}(\mathcal{R})$ and $W^{\mathrm{u}}(0)$. However, the tangent space of the unstable manifold at the origin is given by

$$
T_{0} W^{\mathrm{u}}(0)=\{(A, 0) ; A \in \mathbb{C}\}
$$


so that $\operatorname{Fix}(\mathcal{R})$ and $W^{\mathrm{u}}(0)$ are clearly not transverse for $\varepsilon=0$. Thus, we shall continue the unstable manifold up to $x=0$ and expand it in terms of $\varepsilon$.

We introduce a new variable $\tau$ via

$$
\tau:=\tanh (\kappa x), \quad x=\frac{1}{2 \kappa} \ln \frac{1+\tau}{1-\tau}
$$

where $\kappa>0$ is chosen to be smaller than a fourth of the exponential decay rate of the pulse $q_{1}(x)$. We can then write (5.5) as the smooth autonomous system

$$
\begin{aligned}
A_{x} & =\mathrm{i} A+B+\mathrm{i} A P \\
B_{x} & =\mathrm{i} B+A|A|^{2}+\varepsilon g\left(q_{1}(\tau)\right)+\mathrm{i} B P+A Q+\mathrm{O}\left(|A|^{5}+|B|^{5}+(|A|+|B|)\left|\varepsilon g\left(q_{1}(\tau)\right)\right|\right) \\
\tau_{x} & =\frac{1-\tau^{2}}{\kappa} .
\end{aligned}
$$

The asymptotic system at $x=-\infty$ corresponds now to $\tau=-1$ with the $\tau$-direction being the strong unstable direction within the unstable manifold of the origin. For $\varepsilon=0$, the $(A, B)$-components of (5.7), and therefore also the strong unstable fibers, do not depend on $\tau$. Since the unstable foliation is smooth in $\varepsilon$, we can expand it at $\tau=0$ (i.e. at $x=0$ ) and get

$$
W^{\mathrm{u}}(0)=r \mathrm{e}^{\mathrm{i} \phi}\left(\begin{array}{c}
1 \\
\frac{r}{\sqrt{2}}
\end{array}\right)+\varepsilon\left(\begin{array}{c}
d_{A} \\
d_{B}
\end{array}\right)+\mathrm{O}\left(r^{3}+\varepsilon r+\varepsilon^{2}\right) .
$$

Next, we compute the coefficients $d_{A}, d_{B}$ which are given by the Melnikov integrals

$$
\left(\begin{array}{l}
d_{A} \\
d_{B}
\end{array}\right)=\int_{-\infty}^{0} \mathrm{e}^{-\mathrm{i} x}\left(\begin{array}{rr}
1 & -x \\
0 & 1
\end{array}\right)\left(\begin{array}{c}
0 \\
g\left(q_{1}(x)\right)
\end{array}\right) \mathrm{d} x=\int_{-\infty}^{0} \mathrm{e}^{-\mathrm{i} x}\left(\begin{array}{c}
-x g\left(q_{1}(x)\right) \\
g\left(q_{1}(x)\right)
\end{array}\right) \mathrm{d} x
$$

which involve the perturbation $g$ and the evolution of the linearization of $(5.5)$ about $(A, B)=0$. As we shall see below, we need that

$$
\operatorname{Re} d_{B}=\int_{-\infty}^{0} g\left(q_{1}(x)\right) \cos x \mathrm{~d} x<0
$$

Recall that the pulse achieves its unique positive maximum $M$ at $x=0$. Thus, upon defining $g\left(u_{1}\right)$ by

$$
g\left(u_{1}\right)=\left\{\begin{array}{rl}
-1 & M-\delta_{0}<u_{1} \\
0 & u_{1}<M-2 \delta_{0}
\end{array}\right.
$$

for some $0<\delta_{0} \ll 1$ with $g^{\prime}\left(u_{1}\right) \geq 0$, we can ensure that (5.9) is true.

We are now prepared to find intersections of $W^{\mathrm{u}}(0)$ and $\operatorname{Fix}(\mathcal{R})$ at $\tau=0$ which are given as solutions to the equation

$$
\left(\begin{array}{c}
a \\
\mathrm{i} b
\end{array}\right)=r \mathrm{e}^{\mathrm{i} \phi}\left(\begin{array}{c}
1 \\
\frac{r}{\sqrt{2}}
\end{array}\right)+\varepsilon\left(\begin{array}{c}
d_{A} \\
d_{B}
\end{array}\right)+\mathrm{O}\left(r^{3}+\varepsilon r+\varepsilon^{2}\right)
$$


in $\mathbb{C}^{2}$, where $a, b \in \mathbb{R}, 0 \leq r \ll 1$ and $\phi \in \mathbb{R}$. Decomposing this equation into real and imaginary part, we get

$$
\begin{aligned}
a & =r \cos \phi+\varepsilon \operatorname{Re} d_{A}+\mathrm{O}\left(r^{3}+\varepsilon r+\varepsilon^{2}\right) \\
0 & =r \sin \phi+\varepsilon \operatorname{Im} d_{A}+\mathrm{O}\left(r^{3}+\varepsilon r+\varepsilon^{2}\right) \\
0 & =\frac{r^{2}}{\sqrt{2}} \cos \phi+\varepsilon \operatorname{Re} d_{B}+\mathrm{O}\left(r^{3}+\varepsilon r+\varepsilon^{2}\right) \\
b & =\frac{r^{2}}{\sqrt{2}} \sin \phi+\varepsilon \operatorname{Im} d_{B}+\mathrm{O}\left(r^{3}+\varepsilon r+\varepsilon^{2}\right)
\end{aligned}
$$

The first and fourth equation can be solved for $(a, b)$. To solve the remaining two equations, we scale $(r, \phi)=\sqrt{\varepsilon}(R, \Phi)$ and get

$$
\begin{aligned}
& 0=R \Phi+\operatorname{Im} d_{A}+\mathrm{O}(\sqrt{\varepsilon}) \\
& 0=\frac{R^{2}}{\sqrt{2}}+\operatorname{Re} d_{B}+\mathrm{O}(\sqrt{\varepsilon})
\end{aligned}
$$

Since $\operatorname{Re} d_{B}<0$ by (5.9), we can solve the system for $\varepsilon=0$ and obtain

$$
R_{*}=\sqrt{\sqrt{2}\left|\operatorname{Re} d_{B}\right|}, \quad \Phi_{*}=\frac{-\operatorname{Im} d_{A}}{\sqrt{\sqrt{2}\left|\operatorname{Re} d_{B}\right|}} .
$$

The derivative of the right-hand side of $(5.10)$ at $\left(R_{*}, \Phi_{*}\right)$ is given by

$$
\left(\begin{array}{cc}
\Phi_{*} & R_{*} \\
\sqrt{2} R_{*} & 0
\end{array}\right)
$$

with determinant $-\sqrt{2} R_{*}^{2} \neq 0$. This proves that the solution persists for $\varepsilon>0$ as a regular root and establishes therefore also transversality.

\subsection{Oscillatory instabilities: An example}

We will prove that there are constants $0<\varepsilon \ll 1, m_{1}, m_{2} \in \mathbb{R}$ and a function $g: \mathbb{R} \rightarrow \mathbb{R}$ with $g\left(u_{1}\right)=0$ for $u_{1}$ close to zero such that the system

$$
\begin{aligned}
\partial_{t} u_{1} & =\partial_{x x} u_{1}-u_{1}+u_{1}^{3}+\frac{1}{2} u_{1} u_{2}+v_{1}+v_{2} \\
\partial_{t} u_{2} & =\frac{1}{\delta^{2}}\left[\frac{1}{\delta^{2}} \partial_{x x} u_{2}-12.5 \delta^{2} u_{2}-\frac{1}{2} u_{1}^{2}+c_{0} u_{1}^{2} u_{2}\right] \\
\partial_{t} v_{1} & =\partial_{x x} v_{1}+\left[\mu-v_{1}^{2}-v_{2}^{2}\right] v_{1}-\left[1+\beta\left(v_{1}^{2}+v_{2}^{2}\right)\right] v_{2}+\varepsilon m_{2} g\left(u_{1}^{2}\right) \\
\partial_{t} v_{2} & =\partial_{x x} v_{2}+\left[1+\beta\left(v_{1}^{2}+v_{2}^{2}\right)\right] v_{1}+\left[\mu-v_{1}^{2}-v_{2}^{2}\right] v_{2}+\varepsilon m_{1} g\left(u_{1}^{2}\right)
\end{aligned}
$$

satisfies the assumptions of Theorems 5 and 6 .

For $\varepsilon=0$, the last two equations written in complex notation $A=v_{1}+\mathrm{i} v_{2}$ are

$$
A_{t}=A_{x x}+(\mu+\mathrm{i}) A-(1+\mathrm{i} \beta)|A|^{2} A .
$$


Thus, the critical dispersion relation is given by $\lambda= \pm \mathrm{i}-k^{2}$, and the origin undergoes a generic Hopf bifurcation at $\mu=0$. In particular, Hypotheses 7 and 8 are satisfied. Upon choosing $\beta$ sufficiently close to zero, Proposition 1.2 shows that Hypotheses 9 and 10 are met. These hypotheses continue to be met for $\varepsilon>0$ since $g\left(u_{1}^{2}\right)$ will vanish for all $u_{1}$ close to zero and does therefore not affect the above arguments.

It remains to verify Hypotheses 11 and 12. Note that the equation for $u$ decouples for $\varepsilon=0$. In particular, (5.11) admits the pulse $(u, v)=(q, 0)$, which is still transversely constructed and therefore persists for all $0<\varepsilon \ll 1$ with

$$
(u, v)(\varepsilon)=(q, 0)+\mathrm{O}(\varepsilon)
$$

Next, we denote the linearization of (5.11) at $\mu=0$ about the pulse by $\mathcal{L}_{\varepsilon}$. It is clear from our construction that $\lambda=0$ is a simple eigenvalue of $\mathcal{L}_{\varepsilon}$ and that $\lambda=\mathrm{i} \ell$ is not in the spectrum for all $\ell \in \mathbb{Z} \backslash\{0, \pm 1\}$ provided we choose $\varepsilon>0$ small enough. Thus, it suffices to prove that the null space of $\mathcal{L}_{\varepsilon}-\mathrm{i}$ is trivial in $C^{0}\left(\mathbb{R}, \mathbb{C}^{4}\right)$.

We begin by setting $\varepsilon=0$. We have

$$
\mathcal{L}_{0}-\mathrm{i}=\left(\begin{array}{cc}
\mathcal{L}_{u}-\mathrm{i} & B \\
0 & \mathcal{L}_{v}-\mathrm{i}
\end{array}\right), \quad B=\left(\begin{array}{cc}
1 & 1 \\
0 & 0
\end{array}\right), \quad \mathcal{L}_{v}=\left(\begin{array}{cc}
\partial_{x x} & -1 \\
1 & \partial_{x x}
\end{array}\right)
$$

where $\mathcal{L}_{u}$ is the linearization of (5.1) about the pulse $q$. The eigenvalue problem

$$
\left[\mathcal{L}_{0}-\mathrm{i}\right]\left(\begin{array}{c}
u \\
v
\end{array}\right)=0
$$

has a unique (up to constant multiples) bounded nontrivial solution $\left(u^{*}, v^{*}\right)$ given by

$$
v^{*}=\left(\begin{array}{c}
\mathrm{i} \\
1
\end{array}\right), \quad u^{*}=\left[\mathcal{L}_{u}-\mathrm{i}\right]^{-1} B v^{*}
$$

Note that $\left(\mathcal{L}_{u}-\mathrm{i}\right)$ is invertible on $C^{0}\left(\mathbb{R}, \mathbb{C}^{2}\right)$ since the pulse of (5.1) is stable. In particular, the null space of $\left(\mathcal{L}_{0}-\mathrm{i}\right)$ is not trivial.

For later reference, we note that $u_{1}^{*}(x)$ is even and vanishes at most on a discrete set: Indeed, $u_{1}^{*}$ satisfies

$$
\partial_{x x} u_{1}+\left(3 q_{1}(x)^{2}-1+\frac{1}{2} q_{2}(x)\right) u_{1}+\frac{1}{2} q_{1}(x) u_{2}=1 .
$$

Since $u_{2}$ is bounded and $q_{1}(x) \rightarrow 0$, we see that $u_{1}$ cannot vanish identically. On the other hand, since the pulse $q(x)$ is analytic, so is the solution $u_{1}^{*}(x)$, which proves our claim. We also mention that the unique bounded solution of the adjoint eigenvalue problem $\left[\mathcal{L}_{0}^{*}+\mathrm{i}\right] Q^{*}=0$ is given by

$$
Q^{*}(x)=\left(\begin{array}{l}
u_{1} \\
u_{2} \\
v_{1} \\
v_{2}
\end{array}\right)=\left(\begin{array}{l}
0 \\
0 \\
\mathrm{i} \\
1
\end{array}\right)
$$


Note that the eigenvalue at $\lambda= \pm \mathrm{i}$ lies on the absolute spectrum given by $\lambda= \pm \mathrm{i}-k^{2}$. Such eigenvalues can disappear in edge bifurcations upon adding perturbations [9]. We shall prove that this indeed happens for our system if we choose $\varepsilon>0$ small enough. The idea is to calculate the Evans function associated with the eigenvalue problem (5.13) and to expand it in terms of $\gamma=\sqrt{\lambda-\mathrm{i}}$ and $\varepsilon$. We refer to [9] for the relevant theory that we shall apply below.

The Evans function at $\varepsilon=0$ is simply the Wronskian of an appropriate set of solutions of

$$
\left[\mathcal{L}_{0}-\left(\mathrm{i}+\gamma^{2}\right)\right]\left(\begin{array}{l}
u \\
v
\end{array}\right)=0
$$

which, due to (5.12), is upper triangular. Thus, its Wronskian is the product of the Wronskians for the blocks on the diagonal. We shall only need the determinant of the constant-coefficient problem $\mathcal{L}_{v}-\left(\mathrm{i}+\gamma^{2}\right)$ which, in the coordinates $\left(v, v^{\prime}\right)$, is given by

$$
E(\gamma, 0)=\operatorname{det}\left[\begin{array}{cccc}
\mathrm{i} & \mathrm{i} & -\mathrm{i} & -\mathrm{i} \\
1 & 1 & 1 & 1 \\
\mathrm{i} \gamma & -\mathrm{i} \gamma & -\mathrm{i} \sqrt{2 \mathrm{i}+\gamma^{2}} & \mathrm{i} \sqrt{2 \mathrm{i}+\gamma^{2}} \\
\gamma & -\gamma & \sqrt{2 \mathrm{i}+\gamma^{2}} & -\sqrt{2 \mathrm{i}+\gamma^{2}}
\end{array}\right]=-16 \sqrt{2 \mathrm{i} \gamma}+\mathrm{O}\left(\gamma^{2}\right)
$$

Next, we need to compute the derivative of the Evans function with respect to $\varepsilon$ at $\gamma=0$. The procedure for this computation can be found in $[9, \S 4]$ : The result is that

$$
\partial_{\varepsilon} E(0,0)=\frac{W}{\left|Q^{*}(0)\right|^{2}} \int_{-\infty}^{\infty}\left\langle Q^{*}(x),\left.\partial_{\varepsilon} \mathcal{L}_{\varepsilon}\right|_{\varepsilon=0}\left(\begin{array}{c}
u^{*} \\
v^{*}
\end{array}\right)\right\rangle \mathrm{d} x
$$

where

$$
W=\operatorname{det}\left[\begin{array}{cccc}
0 & \mathrm{i} & -\mathrm{i} & -\mathrm{i} \\
0 & 1 & 1 & 1 \\
\mathrm{i} & -\mathrm{i} \gamma & -\mathrm{i} \sqrt{2 \mathrm{i}+\gamma^{2}} & \mathrm{i} \sqrt{2 \mathrm{i}+\gamma^{2}} \\
1 & -\gamma & \sqrt{2 \mathrm{i}+\gamma^{2}} & -\sqrt{2 \mathrm{i}+\gamma^{2}}
\end{array}\right]=-8(1+\mathrm{i}), \quad\left|Q^{*}(0)\right|^{2}=2
$$

Thus, we need to compute $\left.\partial_{\varepsilon} \mathcal{L}_{\varepsilon}\right|_{\varepsilon=0}$ which is composed of two contributions: the explicit $\varepsilon$ dependence $\varepsilon g\left(u_{1}^{2}\right)\left(0,0, m_{2}, m_{1}\right)$ on the right-hand side of (5.11) and terms coming from the unknown $\varepsilon$-dependence of the persisting pulse. The derivative of the former term is given simply by

$$
2 q_{1}(x) g^{\prime}\left(q_{1}^{2}(x)\right) u_{1}^{*}(x)\left(\begin{array}{c}
0 \\
0 \\
m_{2} \\
m_{1}
\end{array}\right) .
$$

Since the $u$-component of $Q^{*}$ vanishes, we need to account only for the $\varepsilon$-dependence of the pulse in $\mathcal{L}_{v}$. However, the nonlinearity in the $v$-equation is cubic: Since the perturbed pulse is $\mathrm{O}(\varepsilon)$-close to $(q, 0)$, the effect on the linearized operator is actually of order $\mathrm{O}\left(\varepsilon^{2}\right)$, and does therefore not contribute to $\left.\partial_{\varepsilon} \mathcal{L}_{\varepsilon}\right|_{\varepsilon=0}$. In summary, using also that $u_{1}^{*}$ is even, (5.16) becomes

$$
\partial_{\varepsilon} E(0,0)=-16(1+\mathrm{i})\left(m_{1}+\mathrm{i} m_{2}\right) \int_{0}^{\infty} q_{1}(x) g^{\prime}\left(q_{1}^{2}(x)\right) u_{1}^{*}(x) \mathrm{d} x .
$$


We claim that we can find a function $g\left(u_{1}\right)$ that vanishes near $u_{1}=0$ so that

$$
d_{0}:=\int_{0}^{\infty} q_{1}(x) g^{\prime}\left(q_{1}^{2}(x)\right) u_{1}^{*}(x) \mathrm{d} x \neq 0 .
$$

Indeed, we have seen that $u_{1}^{*}$ vanishes at most at a discrete set. Thus, there are numbers $0<x_{*} \ll 1$ and $0<\delta_{1} \ll x_{*}$ so that $q_{1}(x)$ is strictly decreasing on $J:=\left(x_{*}-\delta_{1}, x_{*}+\delta_{1}\right)$ and $u_{1}^{*}(x) \neq 0$ for $x \in J$. Denote by $\check{g}$ the continuous, piecewise linear function

$$
\check{g}\left(u_{1}^{2}\right)=\left\{\begin{array}{cl}
0 & q_{1}\left(x_{*}-\delta_{1}\right) \leq u_{1} \\
\frac{u_{1}-q_{1}\left(x_{*}-\delta_{1}\right)}{q_{1}\left(x_{*}+\delta_{1}\right)-q_{1}\left(x_{*}-\delta_{1}\right)} & q_{1}\left(x_{*}+\delta_{1}\right)<u_{1}<q_{1}\left(x_{*}-\delta_{1}\right) \\
1 & q_{1}(L) \leq u_{1} \leq q_{1}\left(x_{*}+\delta_{1}\right) \\
2 \frac{q_{1}(L)-u_{1}}{q_{1}(L)} & q_{1}(L) / 2<u_{1}<q_{1}(L) \\
0 & 0 \leq u_{1} \leq q_{1}(L) / 2
\end{array}\right.
$$

for some $L \gg 1$, so that $\check{g}^{\prime}$ is piecewise constant:

$$
\check{g}^{\prime}\left(u_{1}^{2}\right)=\left\{\begin{array}{cl}
0 & q_{1}\left(x_{*}-\delta_{1}\right) \leq u_{1} \\
1 & q_{1}\left(x_{*}+\delta_{1}\right)<u_{1}<q_{1}\left(x_{*}-\delta_{1}\right) \\
0 & q_{1}(L) \leq u_{1} \leq q_{1}\left(x_{*}+\delta_{1}\right) \\
1 & q_{1}(L) / 2<u_{1}<q_{1}(L) \\
0 & 0 \leq u_{1} \leq q_{1}(L) / 2
\end{array}\right.
$$

Furthermore, let $\eta_{\delta_{2}}(x)$ be the standard mollifier function (so that $\eta_{\delta}$ converges to the delta-function as $\delta_{2} \rightarrow 0$ ). We then define $g:=\eta_{\delta_{2}} \star \check{g}$ (with $\star$ denoting convolution) and note that $g^{\prime} \rightarrow \check{g}^{\prime}$ in $L^{1}(\mathbb{R})$ as $\delta_{2} \rightarrow 0$. In particular, we see that

$$
d_{0}=\int_{0}^{\infty} q_{1}(x) g^{\prime}\left(q_{1}^{2}(x)\right) u_{1}^{*}(x) \mathrm{d} x \longrightarrow 2 \delta_{1} q_{1}\left(x_{*}\right) u_{1}^{*}\left(x_{*}\right)+\mathrm{O}\left(\delta_{1}^{2}+\mathrm{e}^{-\alpha L}\right)
$$

for some $\alpha>0$ that depends only on the parameters in (5.1), and therefore $d_{0} \neq 0$ for $\delta_{1}, \delta_{2}$ sufficiently small.

Thus, we obtain the expansion

$$
E(\gamma, \varepsilon)=-16 \sqrt{2 \mathrm{i}} \gamma-16(1+\mathrm{i})\left(m_{1}+\mathrm{i} m_{2}\right) d_{0}+\mathrm{O}\left(\gamma^{2}+\varepsilon^{2}\right)
$$

for the Evans function. Its roots are given by

$$
\gamma_{*}(\varepsilon)=\frac{-(1+\mathrm{i}) d_{0}}{\sqrt{2 \mathrm{i}}}\left(m_{1}+\mathrm{i} m_{2}\right) \varepsilon+\mathrm{O}\left(\varepsilon^{2}\right) .
$$

Roots $\gamma$ of the Evans function with $\operatorname{Re} \gamma>0$ correspond to eigenvalues $\lambda=i+\gamma^{2}$ of the operator $\mathcal{L}_{\varepsilon}$, while roots with $\operatorname{Re} \gamma<0$ are resonance poles (the associated "eigenfunctions" grow exponentially). Since $d_{0} \neq 0$, there exist numbers $m_{1}, m_{2} \in \mathbb{R}$ so that $\operatorname{Re} \gamma_{*}<0$ for all $0<\varepsilon \ll 1$. Hence, the null space of $\left(\mathcal{L}_{\varepsilon}-\mathrm{i}\right)$ is trivial for all small enough $\varepsilon>0$, which verifies Hypotheses 11 and 12 . 


\section{Discussion}

We have demonstrated that a branch of standing pulses can be continued in a unique way through parameter values where its background state undergoes a pitchfork or Turing instability. For pitchfork instabilities, the standing pulse selects one of the two equilibria that arise in the pitchfork bifurcation. For Turing instabilities, the only degree of freedom is the choice of the wavenumber of the asymptotic Turing pattern which can be specified arbitrarily in the Eckhaus stable band, while the bifurcating pulses select the phase of the Turing pattern. We emphasize that, beyond the pitchfork or Turing instability, pulses that are asymptotic to the trivial rest state cease to exist.

If the background state undergoes a transcritical bifurcation, $u_{t}=\mu u-u^{2}$, a straightforward adaptation of the methods presented in this paper show that the pulse can again be continued in a unique fashion through the bifurcation point and that it is asymptotic to the unique stable equilibrium that emerges in the transcritical bifurcation. For saddle-node bifurcations, the pulse disappears with the stable equilibrium. Together with the large pulse, an unstable small-amplitude pulse that is asymptotic to the stable equilibrium disappears at the bifurcation point.

Oscillatory instabilities are somewhat different. The primary standing pulse persists as is usual in oscillatory bifurcations. On the other hand, two different oscillatory pulses emerge from this bifurcation, one of which is symmetric, the other one anti-symmetric. We emphasize that the bifurcating oscillatory pulses can be interpreted as sources in that the group velocities (and, in fact, the phase velocities) of the small-amplitude travelling waves at their tails point away from the oscillatory pulse. We refer to [21] for more details and references regarding sources.

\section{Boundary layers and inhomogeneities}

The analysis presented in this paper gives also some insight into phenomena involving boundary layers. Consider, for example, the reaction-diffusion system (1.1) on the half line $x \in \mathbb{R}^{-}$with boundary conditions $b\left(u, u_{x}\right)=0$ at $x=0$ or, more specifically, $u(0, t)=1$, say. If the origin $u=0$ is a stable equilibrium for the reaction-diffusion system on $x \in \mathbb{R}$, the boundary conditions typically introduce a boundary layer $q(x)$ with $b\left(q, q_{x}\right)=0$ so that $q(x) \rightarrow 0$ exponentially as $x \rightarrow-\infty$. If the rest state $u=0$ undergoes a Turing instability, a phenomenon equivalent to Theorem 1 occurs: at criticality, $q(x)$ typically decays only algebraically $q(x)=\mathrm{O}(1 / x)$ while, above onset, all stable Turing patterns coexist with the boundary. The phase of the Turing patterns relative to the boundary is uniquely determined. To see this, we follow the proofs of Theorems 1 and 2 but replace the matching subspace $\operatorname{Fix}(\mathcal{R})$ by the manifold defined by $b(u, v)=0$.

In fact, strongly localized inhomogeneities can be treated in a similar fashion. Stationary localized solutions $q(x)$ to $(1.1)$ for a nonlinearity $f$ that satisfies $f(x, u ; \mu) \rightarrow f(u ; \mu)$ exponentially fast as $|x| \rightarrow \infty$ can be continued through Turing bifurcations but converge then to an arbitrarily prescribed stable Turing pattern.

The situation is different, and in fact easier to handle, for boundary conditions that are compatible with the trivial state (Neumann boundary conditions $u_{x}=0$, for instance, are always compatible) or 
if the inhomogeneity in the medium is weak so that its size is comparable to the bifurcating Turing patterns. Both scenarios are similar to degenerate Turing instabilities where the pulse converges exponentially, and not algebraically, as $|x| \rightarrow \infty$. In this case, the pulse therefore lies in the strong unstable fiber of $u=0$, which makes this bifurcation much easier to analyse as we shall see now.

For simplicity, consider pitchfork bifurcations when the pulse converges exponentially at onset. We can project $\operatorname{Fix}(\mathcal{R}) \cap W^{\mathrm{cu}}(0)$ along the smooth strong unstable fibrations onto $W^{\mathrm{c}}(0)$ which gives a curve $\Gamma_{R}$ in the center manifold. Typically, we expect that this curve intersects the unstable manifold transversely so that $\Gamma_{R}$ reduces to the line $A=0$ in the rescaling chart. Upon varying the parameter $\mu_{1}$ that drives the instability and a parameter $\mu_{2}$ which unfolds the degeneracy, we find two wedges $\left|\mu_{2}\right| \geq C \sqrt{\mu_{1}}$ where precisely one of the two homogeneous equilibria that arise in the local pitchfork bifurcation is selected by the pulse. In the parameter region between these wedges, $\Gamma_{R}$ intersects the small-amplitude heteroclinic orbits that connect the two equilibria on the center manifold. In particular, we obtain pulses that converge to either one of the asymptotic equilibria: these pulses are formed by concatenating the large pulse with part of the small-amplitude layer between the two equilibria.

For Turing instabilities, $\Gamma_{R}$ is given by the complex plane $B=0$ which intersects the stable and unstable manifold of Eckhaus-stable Turing patterns transversely along the defect $A=\tanh (x / \sqrt{2})$.

Thus, we conclude that there exist again wedges in parameter space within which a unique Turing pattern (with prescribed wavenumber) is selected. Outside these wedges, two possible phase selections are possible with a phase difference of approximately $\pi$. We suspect that only one of the two possible phases leads to a stable pulse.

\section{Evans functions versus algebraic decay}

The algebraic relaxation that we typically encounter at onset cannot be encoded as spectral information in $L^{2}$. Indeed, the degeneracy of exponential decay at the bifurcation point corresponds to an eigenvalue in an exponentially weighted space $L_{\eta}^{2}$ with norm

$$
|u|_{L_{\eta}^{2}}^{2}=\int_{\mathbb{R}} \mathrm{e}^{\eta x}|u(x)|^{2} \mathrm{~d} x .
$$

In terms of an Evans-function analysis, this eigenvalue generates a root of an Evans function with the "wrong" splitting between stable and unstable subspaces. A similar condition arises as a robustness criterion for contact defects that are typical elementary defects in oscillatory media which correspond to saddle-node homoclinic orbits in a spatial-dynamics description [20, 21].

\section{Extensions}

Our existence results are also applicable to certain classes of Hamiltonian partial differential equations such as coupled nonlinear Schrödinger equations or semilinear wave equations. Turing instabilities in nonlinear Schrödinger equations correspond to resonances between the frequency of a large pulse and a background wave with nonzero wavenumber. Supercriticality of the Turing 
instability is equivalent to a defocusing nonlinearity for the resonant waves. A detailed description of these phenomena is the object of future work.

We also expect that the results presented here are valid for PDEs with additional symmetries. As an example, we mention the complex cubic-quintic Ginzburg-Landau equation which has a gauge symmetry given by an $S^{1}$-action and which exhibits stable pulses that can undergo Hopf bifurcations [10]. In systems with symmetry, the unstable manifold and the fixed-point space of the relevant reverser will not intersect transversely along pulses with trivial isotropy. However, the direction along the pulse's group orbit can be used to compensate for the lack of transversality: For the gauge symmetry in the Ginzburg-Landau equation, the relative phase between the Nozaki-Bekki holes and the pulse provides the additional free parameter needed to solve the bifurcation equations. We remark that this scenario also occurs in the master-modelocking equation [8]: however, this equation includes nonlocal terms which further complicates the analysis.

\section{A Stability of the Nozaki-Bekki holes in the real CGL limit}

We shall prove Proposition 1.2. First, we remark that the verification of Hypotheses 9 and 10(i) is well known: see, for instance, [7, Theorem 1.1] with $\sigma=0$ and $d_{4}=0$.

It remains to verify Hypothesis 10(ii) for $\alpha, \beta$ sufficiently small. This issue has been investigated in [7], but the proofs of the stability theorems stated in [7] are incorrect due to a number of algebra mistakes: specifically, the linearization [7, (4.5) and (4.6)] is wrong and the sign of $\partial_{\varepsilon}^{6} E(0)$ on [7, Page 104] is incorrect. We give the corrected statements and proofs here. Throughout the remainder of this section, we shall closely follow the notation of [7].

First, [7, (3.22)] shows that the Evans function $E(\gamma)$ has three roots at the origin when $\varepsilon=0$ which corresponds to the real Ginzburg-Landau equation. In addition, the standing hole $\Phi=\tanh (x)$ has one unstable real eigenvalue with even eigenfunction at a fixed distance away from the origin, and there are no other roots of the Evans function in the closed right-half plane. The unstable eigenvalue arises due to Sturm-Liouville theory since $L_{-} \Phi=0$, and $\Phi$ has one zero (the statement at the bottom of [7, Page 96] is therefore wrong). The unstable eigenvalue will persist for $0<\varepsilon \ll 1$

and, since the linearization of the CGL about standing pulses respects the $x \mapsto-x$ symmetry, the associated eigenfunction is even in $x$.

It therefore remains to track the three small roots of the Evans function for $0<\varepsilon \ll 1$. Inspecting [7, Page 87 and Figure 3], we see that the standing holes corresponding to $a>0$ are sources, namely the Nozaki-Bekki holes, in the notation of [21]. Thus, [21, Lemma 4.4] implies that the Evans function of each Nozaki-Bekki hole has at least two roots at the origin with eigenfunctions given by $\left(A_{\mathrm{nb}}^{\prime}, B_{\mathrm{nb}}^{\prime}\right)$ and $\left(\mathrm{i} A_{\mathrm{nb}}, \mathrm{i} B_{\mathrm{nb}}\right)$ : in other words, the roots are enforced by the translational and gauge symmetries of the CGL. Note that the eigenfunction associated with the gauge symmetry is odd, while the translational eigenfunction is even.

It suffices now to calculate the remaining third root. We compute the Evans function for even eigenfunctions by matching decaying solutions at $x=-\infty$ with the fixed-point space $\operatorname{span}\left\{u_{1}(0), u_{4}(0)\right\}$. 
The resulting Evans function can be written as

$$
E(\gamma, \varepsilon)=Y_{\mathrm{s}}^{-}(0 ; \gamma) \wedge Y_{\mathrm{f}}^{-}(0 ; \gamma) \wedge u_{1}(0) \wedge u_{4}(0)
$$

where $Y_{\mathrm{s}}^{-}(0 ; 0)=u_{3}(0)$ and $Y_{\mathrm{f}}^{-}(0 ; 0)=u_{1}(0)$. The key is therefore an expansion of $Y_{\mathrm{f}}^{-}(0 ; \gamma)$. The computations in $[7, \S 4.2$ and $\S 4.4]$ show that

$$
\partial_{\varepsilon}^{4} Y_{\mathrm{f}}^{-}(0 ; 0)=16 a^{2} u_{2}(0)+c_{1} u_{1}(0)+c_{2} u_{3}(0), \quad \partial_{\gamma}^{2} Y_{\mathrm{f}}^{-}(0 ; 0)=-\frac{4}{3} u_{2}(0)+c_{3} u_{1}(0)
$$

where $a=\frac{2}{3}\left(d_{1}+d_{3}\right)^{2}$. Thus, roots of (A.1) satisfy

$$
\frac{2 a^{2}}{3} \varepsilon^{4}-\frac{2}{3} \gamma^{2}=0
$$

and are therefore given by

$$
\gamma= \pm a \varepsilon^{2}
$$

Only roots with $\operatorname{Re} \gamma>0$ correspond to eigenvalues $\lambda$ via

$$
\lambda=\lambda_{\mathrm{bp}}(\varepsilon)+\frac{\gamma^{2}}{2}, \quad \lambda_{\mathrm{bp}}(\varepsilon)=-\frac{1}{2} a^{2} \varepsilon^{4}
$$

which shows that only the root at $\lambda=0$ persists as claimed.

In summary, the Nozaki-Bekki holes near the real Ginzburg-Landau limit have precisely three eigenvalues in the closed right-half plane: One is located at a fixed distance away from the origin, while the other two are locked at the origin. The eigenfunctions of the real unstable eigenvalue and the translational eigenvalue at the origin are even, while the eigenfunction associated with the gauge eigenvalue at the origin is odd. This proves Proposition 1.2.

We end this section with a few additional remarks. First, a similar analysis applies to the standing holes for $a>0$ in the NLS limit as they are also sources: Two of the three roots of the Evans function near zero stay at the origin, while the third one disappears as a resonance pole. In contrast to the real Ginzburg-Landau limit, there are no $\mathrm{O}(1)$-unstable eigenvalues.

The case $a<0$ (see again [7, Page 87 and Figure 3]) is slightly more complicated. As indicated there, the standing waves corresponding to $a<0$ are degenerate sinks in the notation of [21]. The arguments in [21, Lemma 4.4] can be adapted easily to prove that one of the three small roots of the Evans function must stay at the origin.

\section{B Expansions of the transition map near equilibria}

We analyse the transition map near the singular equilibria that we encountered in Sections 2.1 and 2.2. We shall focus on Turing instabilities since it is the more complicated case.

We shall use equation $(2.14)$

$$
\dot{a}_{1}=b_{1}-a_{1} n_{\mathrm{r}}+\mathrm{i} a_{1}|A| \check{P}\left(|A|^{2}, n_{\mathrm{i}}\right)
$$




$$
\begin{aligned}
\dot{b}_{1} & =\left(1-\mu_{1}\right) a_{1}-2 b_{1} n_{\mathrm{r}}+\mathrm{i} b_{1}|A| \check{P}\left(|A|^{2}, n_{\mathrm{i}}\right)+a_{1}|A| \check{Q}\left(|A|^{2}, n_{\mathrm{i}}\right)+|A|^{2 m} \check{R}\left(|A|, a_{1} \mathrm{e}^{\mathrm{i} \varphi}, b_{1} \mathrm{e}^{\mathrm{i} \varphi}\right) \\
\dot{\mu}_{1} & =-2 \mu_{1} n_{\mathrm{r}} \\
|\dot{A}| & =|A| n_{\mathrm{r}} \\
\dot{\varphi} & =|A|^{-1}
\end{aligned}
$$

instead of (2.15). The relevant singular equilibria are given by

$$
p_{\mathrm{s}}^{+}=\left(a_{1}, b_{1}, \mu_{1},|A|\right)=\left(\mathrm{e}^{\mathrm{i} \phi}, \frac{1}{\sqrt{2}} \mathrm{e}^{\mathrm{i} \phi}, 0,0\right), \quad \phi \in \mathbb{R} .
$$

Note that the $\varphi$-component provides a rapidly oscillating forcing in higher-order terms in the first three equations. If we set $|A|=0$ in the equations for $\left(a_{1}, b_{1}, \mu_{1}\right)$, then these equations decouple and have smooth right-hand sides. Furthermore, the linearization of the resulting equation about the singular equilibria has one center and three stable eigenvalues; see (2.17). Near the circle of singular equilibria, we can therefore choose coordinates

$$
v=\left(v_{\mathrm{c}}, v_{\mathrm{s}}\right) \in S^{1} \times \mathbb{R}^{3},
$$

which replace $\left(a_{1}, b_{1}, \mu_{1}\right)$, so that $v_{\mathrm{c}}$ parametrizes the circle of singular equilibria and $v_{\mathrm{s}}$ parametrizes the stable manifold of each singular equilibrium. Next, we rescale time for the full ODE (B.1) to remove the factor $n_{\mathrm{r}}$ in the equation for $|A|$ : this is possible since $n_{\mathrm{r}}$ is close to $1 / \sqrt{2}$ near the singular equilibria. The resulting equation is of the form

$$
\begin{aligned}
v^{\prime} & =g_{1}(v)+|A| g_{2}(v,|A|)+|A|^{2 m} g_{3}\left(v,|A|, \mathrm{e}^{\mathrm{i} \varphi}\right) \\
|A|^{\prime} & =|A| \\
\varphi^{\prime} & =\frac{\sqrt{2}}{\left(1+g_{4}(v)\right)|A|^{2}}
\end{aligned}
$$

for smooth nonlinearities $g_{j}$, where we used that $n_{\mathrm{r}}=\left(1+g_{4}(v)\right) / \sqrt{2}$ for some smooth $g_{4}$ with $g_{4}\left(v_{\mathrm{c}}, 0\right)=0$ for all $v_{\mathrm{c}}$.

First, we show that each singular equilibrium of (B.2) has a smooth unstable manifold parametrized by $|A|$. We have

$$
|A|(x)=|A|_{0} \mathrm{e}^{x}
$$

and

$$
\varphi(x ; v)=\varphi_{0}+\int_{0}^{x} \frac{\sqrt{2} \mathrm{e}^{-2 y}}{|A|_{0}^{2}\left(1+g_{4}(v(y))\right)} \mathrm{d} y
$$

for each given function $v(x)$. Thus, the unstable manifold of the singular equilibrium $\left(v_{\mathrm{c}}^{0}, 0\right)$ of (B.2) can be obtained via a solution $v$ to the fixed-point equation

$$
\begin{aligned}
v(x)= & \left(v_{\mathrm{c}}^{0}, 0\right) \\
& +\int_{-\infty}^{x} \mathrm{e}^{\mathcal{A}(x-y)}\left[g_{1}(v(y))+|A|_{0} \mathrm{e}^{y} g_{2}\left(v(y),|A|_{0} \mathrm{e}^{y}\right)+|A|_{0}^{2 m} \mathrm{e}^{2 m y} g_{3}\left(v(y),|A|_{0} \mathrm{e}^{y}, \mathrm{e}^{\mathrm{i} \varphi(y ; v)}\right)\right] \mathrm{d} y
\end{aligned}
$$

where $\varphi(x ; v)$ has been defined in (B.3) and $\mathcal{A}$ is the linearization of $v^{\prime}=g_{1}(v)$ about the circle of equilibria $v=\left(v_{\mathrm{c}}^{0}, 0\right)$. Note that the ODE $v^{\prime}=g_{1}(v)$ is of the form

$$
v_{\mathrm{c}}^{\prime}=0, \quad v_{\mathrm{s}}^{\prime}=g_{1}^{\mathrm{s}}\left(y_{\mathrm{s}}\right)
$$


due to normal-form symmetry of (B.1) for $|A|=0$, and $\mathcal{A}$ does therefore not depend on $v_{\mathrm{c}}$. We record that

$$
\left\|\mathrm{e}^{\mathcal{A} y}\right\| \leq C, \quad y \geq 0
$$

We want to consider (B.4) as a fixed-point equation for $v$ in a small ball centered at zero in $C^{0}\left(\mathbb{R}^{-}\right)$ and solve it using the contraction-mapping principle. Using (B.5), we see that, for any fixed small $|A|_{0}>0$, the right-hand side of (B.4) maps a small ball $\mathcal{B}$ in $C^{0}\left(\mathbb{R}^{-}\right)$into itself. Furthermore, using (B.3), it is straightforward to prove that the right-hand side of (B.4) is $C^{m-1}$ in $\left(v, \varphi_{0}, v_{\mathrm{c}}^{0}\right)$ : the factor $\mathrm{e}^{2 m y}$ in front of $g_{3}$ takes care of the exponential growth $\mathrm{e}^{-2 y}$ of $\varphi$ when taking derivatives with respect to $v$ and $\varphi_{0}$. Moreover, the Lipschitz constant of the right-hand side is smaller than one provided we choose $|A|_{0}>0$ small enough. Thus, we can solve (B.4) uniquely for $v$ which gives the desired unstable manifold.

Next, we flatten the smooth unstable manifolds of each singular equilibrium and obtain a system of the form

$$
\begin{aligned}
v_{\mathrm{u}}^{\prime} & =v_{\mathrm{u}} \\
v_{\mathrm{s}}^{\prime} & =\mathcal{A}_{\mathrm{s}} v_{\mathrm{s}}+g_{\mathrm{u}}^{\mathrm{s}}(v)\left[v_{\mathrm{s}}, v_{\mathrm{u}}\right]+g_{\mathrm{s}}^{\mathrm{s}}(v)\left[v_{\mathrm{s}}, v_{\mathrm{s}}\right]+g_{\varphi}^{\mathrm{s}}\left(v, \mathrm{e}^{\mathrm{i} \varphi}\right)\left[v_{\mathrm{s}}, v_{\mathrm{u}}^{2 m}\right] \\
v_{\mathrm{c}}^{\prime} & =g^{\mathrm{c}}(v)\left[v_{\mathrm{s}}, v_{\mathrm{u}}\right]+g_{\varphi}^{\mathrm{c}}\left(v, \mathrm{e}^{\mathrm{i} \varphi}\right)\left[v_{\mathrm{s}}, v_{\mathrm{u}}^{2 m}\right] \\
\varphi^{\prime} & =\frac{\sqrt{2}}{(1+\check{g}(v)) v_{\mathrm{u}}^{2}}
\end{aligned}
$$

where $v=\left(v_{\mathrm{u}}, v_{\mathrm{s}}, v_{\mathrm{c}}\right)$. Indeed, the quadratic terms in the center direction are a consequence of Fenichel's normal form [6] and the fact that the vector field in the center direction vanishes for $v_{\mathrm{u}}=|A|=0$. The normal-form symmetry of (2.15) with respect to the phase symmetry (2.13) implies that the matrix $\mathcal{A}_{\mathrm{s}}=\operatorname{diag}(-2,-3,-4)$ does not depend on $v_{\mathrm{c}}$. The factor $v_{\mathrm{s}}$ in the equation for $v_{\mathrm{s}}$ arises since the center-unstable manifold is flat.

To analyse (B.6), we proceed as above. For a given constant $T \gg 1$ and bounded continuous functions $\left(v_{\mathrm{s}}, v_{\mathrm{c}}\right)(x)$ for $0 \leq x \leq T$, we have

$$
v_{\mathrm{u}}(x)=\mathrm{e}^{x-T} v_{\mathrm{u}}^{1}, \quad \varphi(x ; v)=\varphi_{0}+\int_{0}^{x} \frac{\sqrt{2}}{(1+\check{g}(v(y))) v_{\mathrm{u}}(y)^{2}} \mathrm{~d} y .
$$

The equations for the remaining variables can be put into the equivalent integral formulation

$$
\begin{aligned}
v_{\mathrm{s}}(x)= & \mathrm{e}^{\mathcal{A}_{\mathrm{s}} x} v_{\mathrm{s}}^{0}+\int_{0}^{x} \mathrm{e}^{\mathcal{A}_{\mathrm{s}}(x-y)}\left(g_{\mathrm{u}}^{\mathrm{s}}(v(y))\left[v_{\mathrm{s}}(y), v_{\mathrm{u}}(y)\right]+g_{\mathrm{s}}^{\mathrm{s}}(v(y))\left[v_{\mathrm{s}}(y), v_{\mathrm{s}}(y)\right]\right. \\
& \left.+g_{\varphi}^{\mathrm{s}}\left(v(y), \mathrm{e}^{\mathrm{i} \varphi(y ; v)}\right)\left[v_{\mathrm{s}}(y), v_{\mathrm{u}}^{2 m}(y)\right]\right) \mathrm{d} y \\
v_{\mathrm{c}}(x)= & v_{\mathrm{c}}^{0}+\int_{0}^{x}\left(g^{\mathrm{c}}(v(y))\left[v_{\mathrm{s}}(y), v_{\mathrm{u}}(y)\right]+g_{\varphi}^{\mathrm{c}}\left(v(y), \mathrm{e}^{\mathrm{i} \varphi(y ; v)}\right)\left[v_{\mathrm{s}}(y), v_{\mathrm{u}}^{2 m}(y)\right]\right) \mathrm{d} y .
\end{aligned}
$$

Arguing as above, we see that there are small constants $\delta_{0}, \delta_{1}>0$ such that the right-hand side of (B.7) is a smooth contraction in the $\delta_{1}$-neighborhood of the origin in the space $C^{0}([0, T])$ with norm

$$
\|\check{v}\|:=\sup _{0 \leq x \leq T}\left[\mathrm{e}^{2 x}\left|v_{\mathrm{s}}(x)\right|+\left|v_{\mathrm{c}}(x)\right|\right]
$$


The contraction constant is uniform in $T$ and $v_{\mathrm{bc}}:=\left(v_{\mathrm{u}}^{1}, v_{\mathrm{s}}^{0}, v_{\mathrm{c}}^{0}, \varphi_{0}\right)$ with $\left|v_{\mathrm{bc}}\right|<\delta_{0}$. In particular, we can solve (B.7) uniquely for each $T \gg 1$ and each given $v_{\mathrm{bc}}$ with norm less than $\delta_{0}$, and the resulting solution is smooth in $v_{\mathrm{bc}}$. Utilizing the norm, we see that

$$
\begin{aligned}
v(0) & =\left(\mathrm{e}^{-T} v_{\mathrm{u}}^{1}, v_{\mathrm{s}}^{0}, v_{\mathrm{c}}^{0}\right) \\
v(T) & =\left(v_{\mathrm{u}}^{1}, \mathrm{O}\left(\mathrm{e}^{-2 T}\right), v_{\mathrm{c}}^{0}+\mathrm{O}\left(\mathrm{e}^{-T}\right)\right) .
\end{aligned}
$$

We shall now exploit (B.8). Recall that we are interested in the unstable manifold of each of the wave equilibria.

Note that these manifolds are smoothly fibered over the artificial phase variable $\varphi_{0}$. We may therefore restrict to an arbitrary but fixed value of $\varphi_{0}$ in the sequel. The unstable manifold of a wave equilibrium then intersects the sphere $\left|v_{\mathrm{s}}\right|=\delta$ in a circle

$$
\left(v_{\mathrm{u}}, v_{\mathrm{s}}, v_{\mathrm{c}}\right)=\left(h_{\mathrm{u}}\left(v_{\mathrm{c}}\right), h_{\mathrm{s}}\left(v_{\mathrm{c}}\right), v_{\mathrm{c}}\right)
$$

where the functions $h_{\mathrm{u}}$ and $h_{\mathrm{s}}$ are smooth. To locate the position of the shooting manifold after the passage near the circle of singular equilibria, we need to satisfy the boundary conditions

$$
v_{\mathrm{u}}(T)=\delta, \quad v_{\mathrm{u}}(0)=h_{\mathrm{u}}\left(v_{\mathrm{c}}\right)
$$

which, upon using (B.8), determines the time-of-flight

$$
T=-\log h_{\mathrm{u}}\left(v_{\mathrm{c}}\right)
$$

The expressions for $v_{\mathrm{c}}(T)$ and $T$ show that the unstable manifold of the wave equilibrium after the passage near the singular equilibrium is again a graph over $v_{\mathrm{c}}$.

The distance of the unstable manifold of a wave equilibrium from the unstable manifold of the singular equilibrium measured at distance $\delta$ away from the singular equilibrium is therefore given by

$$
\left|v_{\mathrm{s}}(T)\right|=\mathrm{O}\left(\mathrm{e}^{-2 T}\right)=\mathrm{O}\left(\left|h_{\mathrm{u}}\left(v_{\mathrm{c}}\right)\right|^{2}\right) .
$$

Note that we exploited here that $\lambda_{\mathrm{s}}=-2 \lambda_{\mathrm{u}}$. Lastly, as for pitchfork bifurcations, we have that $h_{\mathrm{u}}\left(v_{\mathrm{c}}\right)=\mathrm{O}(\sqrt{\mu})$, and we conclude that

$$
\left|v_{\mathrm{s}}(T)\right|=\mathrm{O}(\mu)
$$

as needed.

We remark that a similar result has been established simultaneously and independently by Schecter [24].

Acknowledgments B. Sandstede was partially supported by the Alfred P Sloan Foundation and by the NSF through grant DMS-0203854. A. Scheel was partially supported by the NSF through grant DMS-0203301. 


\section{References}

[1] I.S. Aranson and L. Kramer. The world of the complex Ginzburg-Landau equation. Rev. Mod. Phys. 74 (2002) 99-143.

[2] B. Deng. The Sil'nikov problem, exponential expansion, strong $\lambda$-lemma, $C^{1}$-linearization, and homoclinic bifurcation. J. Differ. Eqns. 79 (1989) 189-231.

[3] A. Doelman, G.M. Hek and N.J.M. Valkhoff. Stabilization by slow diffusion in a real Ginzburg-Landau system. J. Nonlinear Sci. 14 (2004) 237-278.

[4] F. Dumortier. Techniques in the theory of local bifurcations: Blow-up, normal forms, nilpotent bifurcations, singular perturbations. In: "Bifurcations and Periodic Orbits of Vector Fields" (D. Schlomiuk, ed.). Kluwer, Dordrecht, NATO ASI Ser. C 408 (1993) 19-73.

[5] C. Elphick, E. Tirapegui, M.E. Brachet, P. Coullet and G. Iooss. A simple global characterization for normal forms of singular vector fields. Physica D 29 (1987) 95-127.

[6] N. Fenichel. Geometric singular perturbation theory for ordinary differential equations. J. Differ. Eqns. 31 (1979) 53-98.

[7] T. Kapitula and J. Rubin. Existence and stability of standing hole solutions to complex GinzburgLandau equations. Nonlinearity 13 (2000) 77-112.

[8] T. Kapitula, N. Kutz and B. Sandstede. The Evans function for nonlocal equations. Indiana Univ. Math. J. (accepted).

[9] T. Kapitula and B. Sandstede. Stability of bright solitary-wave solutions to perturbed nonlinear Schrödinger equations. Physica D 124 (1998) 58-103.

[10] T. Kapitula and B. Sandstede. Instability mechanism for bright solitary-wave solutions to the cubicquintic Ginzburg-Landau equation. J. Opt. Soc. Amer. B 15 (1998) 2757-2762.

[11] J. Lega. Traveling hole solutions of the complex Ginzburg-Landau equation: A review. Physica D 152/153 (2001) 269-287.

[12] A. Mielke. The Ginzburg-Landau equation in its role as a modulation equation. In: "Handbook of Dynamical Systems II" (B. Fiedler, ed.). North-Holland, Amsterdam, 2 (2002) 759-834.

[13] J.-J. Perraud, A. De Wit, E. Dulos, P. De Kepper, G. Dewel and P. Borckmans. One-dimensional "spirals": Novel asynchroneous chemical wave sources. Phys. Rev. Lett. 71 (1993) 1272-1275.

[14] B. Sandstede. Stability of multiple-pulse solutions. Trans. Amer. Math. Soc. 350 (1998) 429-472.

[15] B. Sandstede. Stability of travelling waves. In: Handbook of Dynamical Systems II (B. Fiedler, ed.). North-Holland (2002) 983-1055.

[16] B. Sandstede and A. Scheel. Essential instability of pulses and bifurcations to modulated travelling waves. Proc. Roy. Soc. Edinburgh A 129 (1999) 1263-1290.

[17] B. Sandstede and A. Scheel. Spectral stability of modulated travelling waves bifurcating near essential instabilities. Proc. Roy. Soc. Edinburgh A 130 (2000) 419-448. 
[18] B. Sandstede and A. Scheel. Absolute and convective instabilities of waves on unbounded and large bounded domains. Physica D 145 (2000) 233-277.

[19] B. Sandstede and A. Scheel. Essential instabilities of fronts: Bifurcation and bifurcation failure. Dynamical Systems 16 (2001) 1-28.

[20] B. Sandstede and A. Scheel. Evans function and blow-up methods in critical eigenvalue problems. Discrete Cont. Dynam. Systems 10 (2004) 941-964.

[21] B. Sandstede and A. Scheel. Defects in oscillatory media: toward a classification. SIAM J. Appl. Dynam. Systems 3 (2004) 1-68.

[22] B. Sandstede, A. Scheel and C. Wulff. Dynamics of spiral waves on unbounded domains using centermanifold reduction. J. Differ. Eqns. 141 (1997) 122-149.

[23] B. Sandstede, A. Scheel and C. Wulff. Bifurcations and dynamics of spiral waves. J. Nonlinear Sci. 9 (1999) 439-478.

[24] S. Schecter. Existence of Dafermos profiles for singular shocks. Preprint (2003).

[25] A. Scheel. Radially symmetric patterns of reaction-diffusion systems. Mem. Amer. Math. Soc. 165 (2003).

[26] G. Schneider. Error estimates for the Ginzburg-Landau approximation. Z. Angew. Math. Physik 45 (1994) 433-457.

[27] A. Vanderbauwhede. Centre manifolds, normal forms and elementary bifurcations. Dynamics reported 2 (1989) 89-169.

[28] T. Wagenknecht. Homoclinic bifurcations in reversible systems. PhD Thesis, TU Ilmenau, 2003.

[29] T. Wagenknecht and A.R. Champneys. When gap solitons become embedded solitons: A generic unfolding. Physica D 177 (2003) 50-70. 